\title{
USERS' GUIDE \\ FOR THE \\ ACCELERATED LEACH TEST \\ COMPUTER PROGRAM
}

Mark Fuhrmann, John H. Heiser, Richard Pietrzak,

Eena-Mai Franz and Peter Colombo

November 1990

RADIOLOGICAL SCIENCES DIVISION

DEPARTMENT OF NUCLEAR ENERGY

BROOKHAVEN NATIONAL LABORATORY, ASSOCIATED UNIVERSITIES, INC.

UPTON, NEW YORK 11973

Prepared for the

NATIONAL LOW-LEVEL WASTE MANAGEMENT PROGRAM

UNITED STATES DEPARTMENT OF ENERGY 


\section{DISCIAIMER}

This report was prepared as an account of work sponsored by an agency of the United States Government. Neither the Inited States Government nor any agency thereof nor any of their employees, nor any of their contractors, subcontractors, or their employees, makes any warranty, express or implied, or assumes any legal liability or responsibility for the accuracy, completeness, or usefulness of any information apparatus, product, or process disclosed, or represents that its use would not infringe privately owned rights. Reference herein to any specific commercial product, process. or service by trade name, trademark, manufacturer, or otherwise, does not necessarily constitute or imply its endorsement, recommendation, or favoring by the United States Government or any agency, contractor or subcontractor thereof. The views and opinions of authors expressed herein do not necessarily state or reflect those of the United States Government or any agency, contractor or subcontractor thereof.

Printed in the United States of America

Available from

National Technical Information Service

U.S. Department of Commerce

5285 Port Royal Road

Springfield, VA 22161

NTIS price codes:

Printed Copy: A09; Microfiche Copy: A01 


\section{ACKNOWLEDGEMENTS}

The authors thank Paul Kalb and Edward Kaplan of Brookhaven National Laboratory for reviewing this document and making many helpful suggestions. We also thank Claudio Pescatore for his guidance and Patricia Durcan for preparing the manuscript. This work was funded by the Department of Energy Low-Level Waste Management Program. 


\begin{abstract}
This report is a step-by-step guide for the Accelerated Leach Test (ALT) Computer Program developed to accompany a new leach test for solidified waste forms. The program is designed to be used as a tool for performing the calculations necessary to analyze leach test data, a modeling program to determine if diffusion is the operating leaching mechanism (and, if not, to indicate other possible mechanisms), and a means to make extrapolations using the diffusion models. The ALT program contains four mathematical models that can be used to represent the data. The leaching mechanisms described by these models are:

1) diffusion through a semi-infinite medium (for low fractional releases),

2) diffusion through a finite cylinder (for high fractional releases),

3) diffusion plus partitioning of the source term,

4) solubility limited leaching.
\end{abstract}

Results are presented as a graph containing the experimental data and the best-fit model curve. Results can also be output as LOTUS 1-2-3 files. 


\section{TABLE OF CONTENTS}

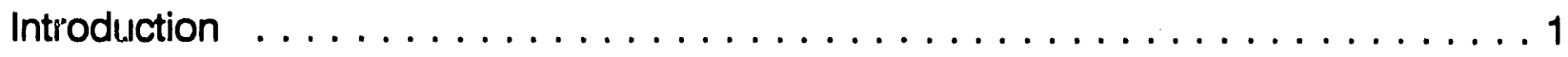

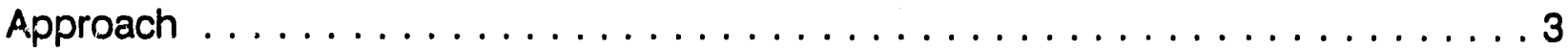

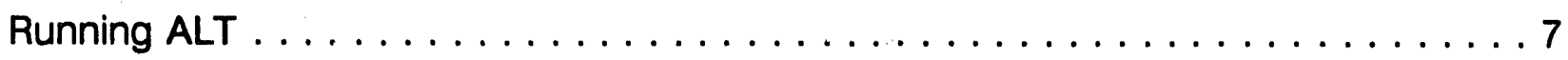

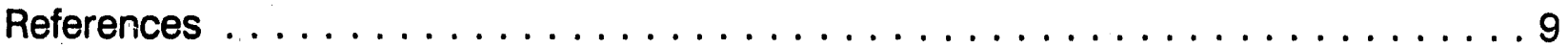

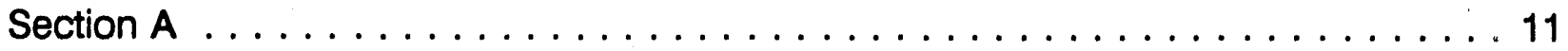

Enter New Data

Multiple Source Term Data

Section B . . . . . . . . . . . . . . . . . . . . . . . 29

Enter New Data

Single Source Term Data

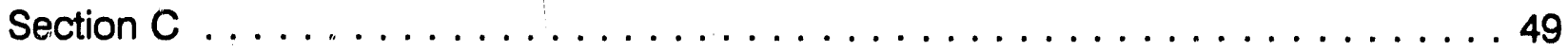

Enter Data as CFL vs Time

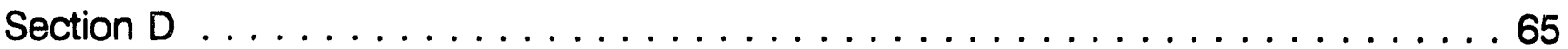

Retrieve a Data Set

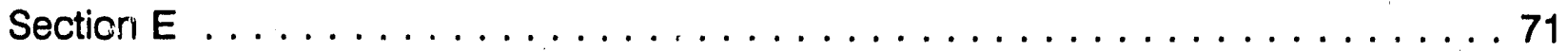

Store a Data Set

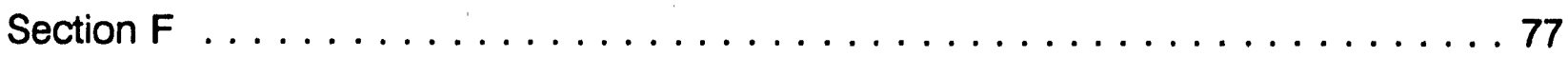

Print a Final Data Set

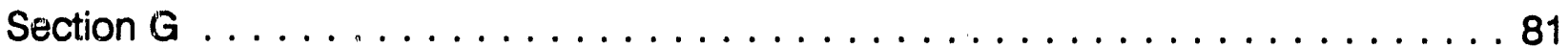

Edit Data

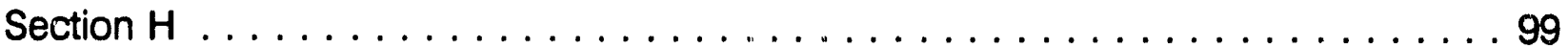

To Add Data to a File

Section I . . . . . . . . . . . . . . . . . . . . . . . . . . . 125

Plot Data

Section $\mathrm{J}$

Plot Comparison 


\section{TABLE OF CONTENTS (Continued)}

Section K . . . . . . . . . . . . . . . . . . . . . . . . . . . . . . . . . 135

Determine $D_{\bullet}$ at Temp.

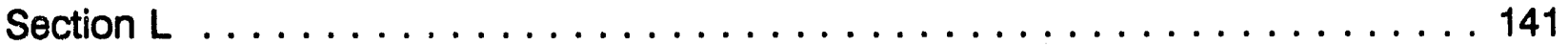

Extend Modeled Curve

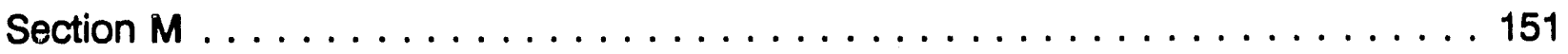

Exit Program

Appendix A........................... A1

Theory

Appendix B .................................. B1

Glossary 


\section{INTRODUCTION}

Determining mechanisms and rates of leaching from radioactive and hazardous waste is one means of assessing the long-term acceptability of waste forms. The long-term integrity of the waste form in its disposal environment is an attribute that is linked to leaching and is important for reliably predicting disposal site behavior. In this way leaching studies are a key element in understanding the processes that lead to disposal site failure. This knowledge, in turn, can ba used to develop ways of preventing releases. Moreover, leaching is a component of degradation processes of many structural materials considered for engineered disposal units. Studies of mechanisms that transport water through these barriers are closely related to studies of leaching mechanisms.

Making pred:-tions of releases from solidified waste forms, that extend to the time periods needed, requires an understanding of the processes of leaching, including the ability to describe those processes with mathematical models. The use of mechanistically based models, as opposed to semi-empirical or curve-fitting routines, is a critical pathway to producing reliable extrapolations of releases to long times. Coupled to the use of mechanistic models is the need to have leaching data that was generated with procedures that adhere (to a practical extent) to the boundary conditions imposed by the models. Particularly important in this context is maintaining relatively low concentrations of dissolved species in the leaching solution throughout the experiment. The maximum leach rate, an intrinsic property of the material tested, can be determined only with optimum test conditions. The Accelerated Leach Test (ALT) method and its associated "ALT" somputer program (which is described in this report) are attempts to link a physical test method with a set of mechanistic models that can be used to interpret the test results. In many cases, this information can then be used to make, within certain constraints, long-term predictions. 


\section{APPROACH}

\section{An Overview of the Accelerated Leach Test}

A leach test is used to determine the quantity of a species of interest that is transported from a solid phase to a liquid phase (the leachant) during a defined period of time. The Accelerated Leach Test is a semi-dynamic test; that is, the leachant is sampled and replaced periodically. Elevated temperature, large leachant volume, and small, solid sample size are used to obtain releases that are accelerated relative to other standard leach tests and to the leaching of full-scale waste forms. The test method is applicable to any material that does not degrade, deform, or change its leaching mechanism at the temperatures used in the test. Detailed instructions for carrying out the Accelerated Leach Test are given in BNL-52268 [1].

\section{An Overview of the ALT Program}

The ALT computer program was developed to accompany the accelerated leach test method. It is designed to be used as:

1) a tool for performing the calculations necessary to analyze leach test data and

2) a modeling program to identify leaching mechanisms and to make extrapolations using the models contained in the program. 
Data is manually input into the program along with the parameters needed to perform the calculations. Many of these parameters (e.g. sample size, time intervals) have default values, wirich are those specified by the accelerated leach test method. However, numbers other than the default values can be input if required. The program produces results as tables of data and as graphs. Results can be saved as LOTUS 1-2-3 files which, in turn, can be imported into commercial graphics programs to produce publication-quality output.

Results of the calculations are presented in several ways. The most basic value determined from a leach test is the Incremental Fraction Leached (IFL) from which the Cumulative Fraction Leached (CFL) is calculated. The CFL is typically plotted as a function of time and ALT does this, when requested, at the end of the calculation sequence. The effective diffusion coefficient $\left(D_{0}\right)$, the partitioning factor $(P)$ or the average concentration can be calculated from the CFL results depending on the model that best represents the data. The activation energy $\left(E_{\mathrm{a}}\right)$ can be determined by ALT when experiments have been run at several different temperatures. The goodness-offit of a model to the data is determined by a sum of the residuals method expressed as a percentage of CFL. This quantity is called $E_{R}$ and its value is used to determine if the model describes the data adequately.

The ALT program contains four mathematical models that can be used to represent the data. The leaching mechanisms described by these models are:

1) diffusion through a semi-infinite medium (for low CFL),

2) diffusion through a finite cylinder (for high CFL),

3) diffusion plus partitioning of the source term, and

4) solubility limited leaching.

These mechanisms were cbserved in studies with various materials during development of the test method [2]. Theoretical background for each mechanism is given in Appendix A. 
Results of the leach test can be extrapolated to long times if the data can be effectively modeled by the diffusion mechanism. The computer program plots both the experimental data and a curve, calculated from the selected model, that best fits the data. For models containing the diffusion mechanism, this is done through an iterative method that optimizes the fit to the entire data curve. If the goodness-of-fit between the data curve and the model gives an $E_{R}$ value that is less than $0.5 \%$, then the model is taken to represent the leaching mechanism of that material. In this case, the model can be used to project releases to long times. In tha case of diffusion, projections can also be made for full size cylinders. If the value of $E_{\mathrm{A}}$ is greater than $0.5 \%$, then the model cannot be used to make projections.

The dissolution model is based on the concept that the concentration of the species of interest in the leachate (and thierefore, the incremental fraction leached) should be the same at the end of cach sampling interval. The mean of the IFL, the standard deviation and the coefficient of variation about the mean are calculated. If the coefficient of variation is less than $10 \%$, then the dissolution model is appropriate.

Results of the ALT program are presented in several forms. Tables of data and associated parameters (e.g. diffusion coefficient and $E_{R}$ ) are displayed on the screen and can be printed. Graphs of CFL plotted as a function of time are generated onscreen and contain both the experimental data points and the curve produced by the model. In addition, graphs are available in which the experimental data is plotted on the $x$-axis and values generated by the model are plotted on the $y$-axis. This type of plot alloivs easy comparison of the relationship botween the data and the model results. If the test has been run at three or more temperatures, the activation energy can be determined by the program. Projections of future releases and for full-scale waste forms can be made if the leaching mechanism is found to be diffusion. 


\section{RUNNING ALT}

\section{Equipment Needed to Run ALT}

The ALT computer program is a compiled version of a program written in Turbo Basic. It will run on IBM or IBM compatible personal computers and requires DOS 2.0 or better. The program uses ASCII code and therefore is compatible with any printer. A math co-processor is desirable to reduce the computation time. A graphics board is required to generate plots and can be a CGA, VGA, EGA or a Hercules color or monocolor board. Without a compatible graphics board the program will do all calculations and list the results but will not be able to produce any plots.

Hard copies of graphs and tables can be obtained by several methods. The user can store data in a .wks type file, which is used by LOTUS 1-2-3. The user can also generate graphs with LOTUS 1-2-3, or with other commercial graphics programs that use the same file type. For low resolution but quick graphics, it is convenient to use "screen grabber" programs. These can be obtained elsewhere and must be compatible to the user's system.

\section{Installing ALT}

The ALT program requires approximately 150 kilobytes of memory, and it is on a 5.25-inch floppy diskette. To run the program, install the diskette in the proper drive and type ALT. The MAIN MENU will then appear on the screen; for help, press F1. 
A glossary of terms can be found at the back of the leach test method along with an appendix that contains details of the models used in the program. The glossary can also be found by pressing F1.

\section{Using the ALT Program}

The remainder of this report consists of detailed instructions on using the ALT program. The report is divided into sections, from $A$ through $M$, which are based on various functions that the program performs. At the beginning of Sections $A, B, C, G$ and $\mathrm{H}$ flow charts are provided of the functions described in that section. Each section is made up of a series of individual screens, that are displayed on the computer during the course of running the program. These screens, which are presented one per page, are each divided into two parts. The first is a reproduction of the contents of the computer screen, and the second is an explanation of what the user must do, or what the computer is doing, at that point. 
REFERENCES

1. Fuhrmann, M., Heiser, J., Pietrzak, R., Franz, E.M., and Colombo, P., Method for Accelerated Leaching of Solidified Waste, BNL - 52268, Brookhaven National Laboratory, Upton, NY 11973, (October 1990).

2. Fuhrmann, M., Pietrazk, R., Heiser, J., Franz, E.M., and P. Colombo, The effects of Temperature on the Leaching Behavior of Cement Waste Forms - The Cement/Sodium Sulfate System, BNL-43449, In: Scientific Basis for Nuclear Waste management XIII, Oversby, V. and Brown, P. (editors), Materials Research Society, Pittsburgh, PA, pages 75-8C, 1990.

$9 / 10$ 


\section{SECTION A}

ENTER NEW DATA

\section{MULTIPLE SOURCE TERM DATA}




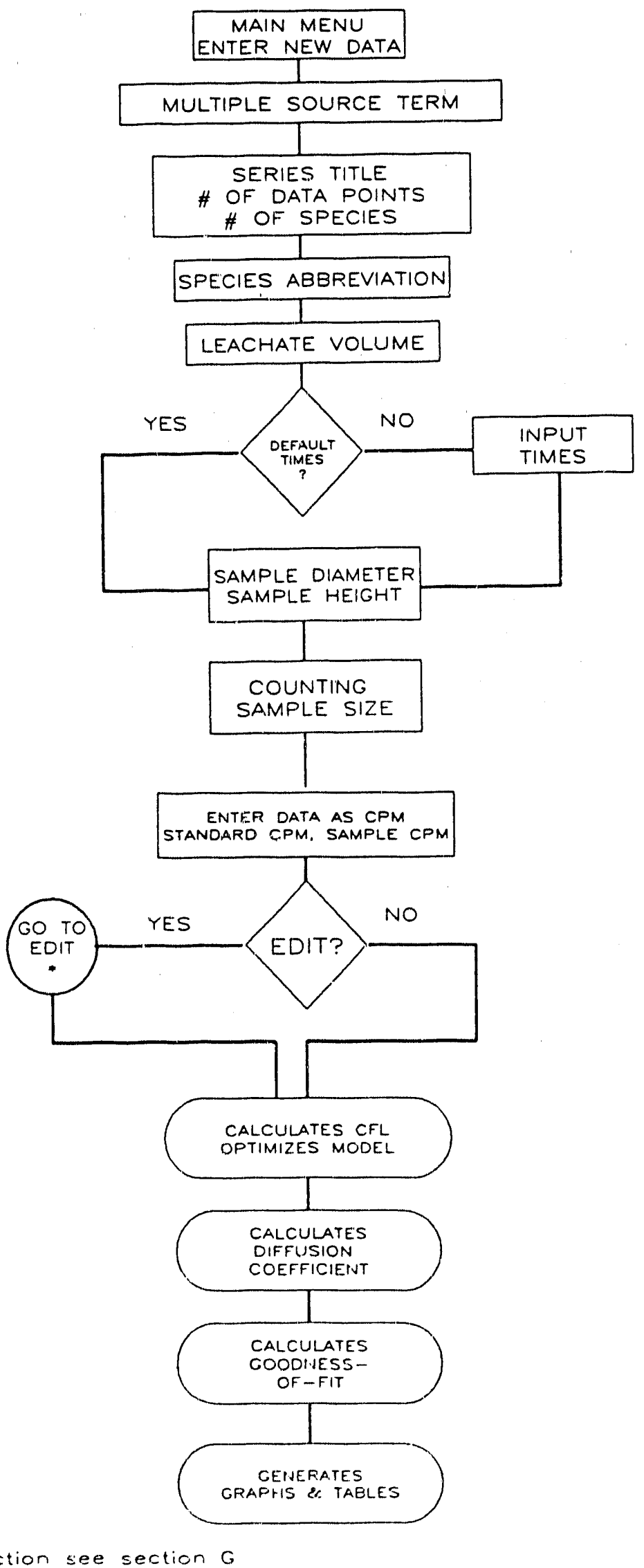




\section{SCREEN 1A}

Use the cursor keys to highlight your choice, then press enter to choose it.

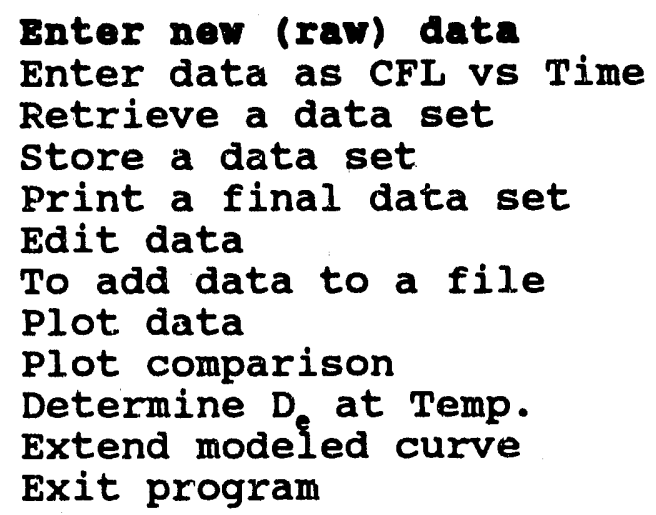

Developed for

DOE National Low-Level Waste Management Program by

Brookhaven National Laboratory

Low-Level Waste Research Group

Upton, N.Y. 11973

Press F1 for help and definitions

\section{EXPLANATION}

This is the Main Menu; from it you can select a variety of functions. Most functions automatically return the user to the Main Menu when they are completed. Press F1 for on-screen help and definitions.

Enter new (raw) data is used to input data in the form of experimental results (e.g., counts per minute or concentration). A variety of other inputs are required, such as height and diameter of the solid sample, volume of leachant, and the number of intervals used. 


\section{SCREEN 2A}

Multiple source term data . . . . . Enter 1 Single source term data . . . . . . Enter 2

To exit and return to main menu .... . Enter 3 Enter your choice

\section{EXPLANATION}

Multiple Source Term Data - Some data require a new value for the source term for each interval. Updating the source term is necessary for very short half-life radionuclides. The source term value that is input here is the number of counts per minute observed from a standard. Corrections for dilutions are made automatically. During the data entry process standard counts are separated by a comma from leachate counts.

If you entered 1, turn to Screen $3 A$ in this section. If you entered 2, turn to Screen $1 B$ in the next section. 


\section{SCREEN 3A}

Enter SERIES TITLE (max 40 characters) +

\section{EXPLANATION}

Type in the title that will be used to identify the data set being entered. A maximum of 40 characters is allowed; you cannot type beyond the plus sign.

This title will be used on tavies and graphs that are produced from this data and will help identify data files that will be stored. 
Enter \# of Sampling Increments (max 50)

\section{EXPLANATION}

Enter the number of sampling increments in the experiment. The standard test has 13 increments.

The program automatically numbers data as it is input, according to the sampling increments. 


\section{SCREEN 5A}

Enter \# of SPECIES (IE Sr, Cs, Co etc) (max 12)

\section{EXPLANATION}

This input is the number of elements or radionuclides analyzed in each set of leachate samples that need to be addressed by the program. A maximum of twelve species are allowed in each data file. 


\section{SCREEN 6A}

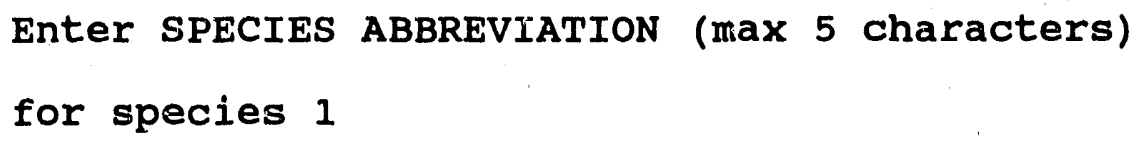

\section{EXPLANATION}

Species abbreviations are letters, numbers or combinations of the two that are used to identify each of the elements or radionuclides to be considered by the program.

Enter the abbreviation for each of the species as the species number changes in the prompt on the screen. A maximum of 5 characters is allowed. 


\section{SCREEN 7A}

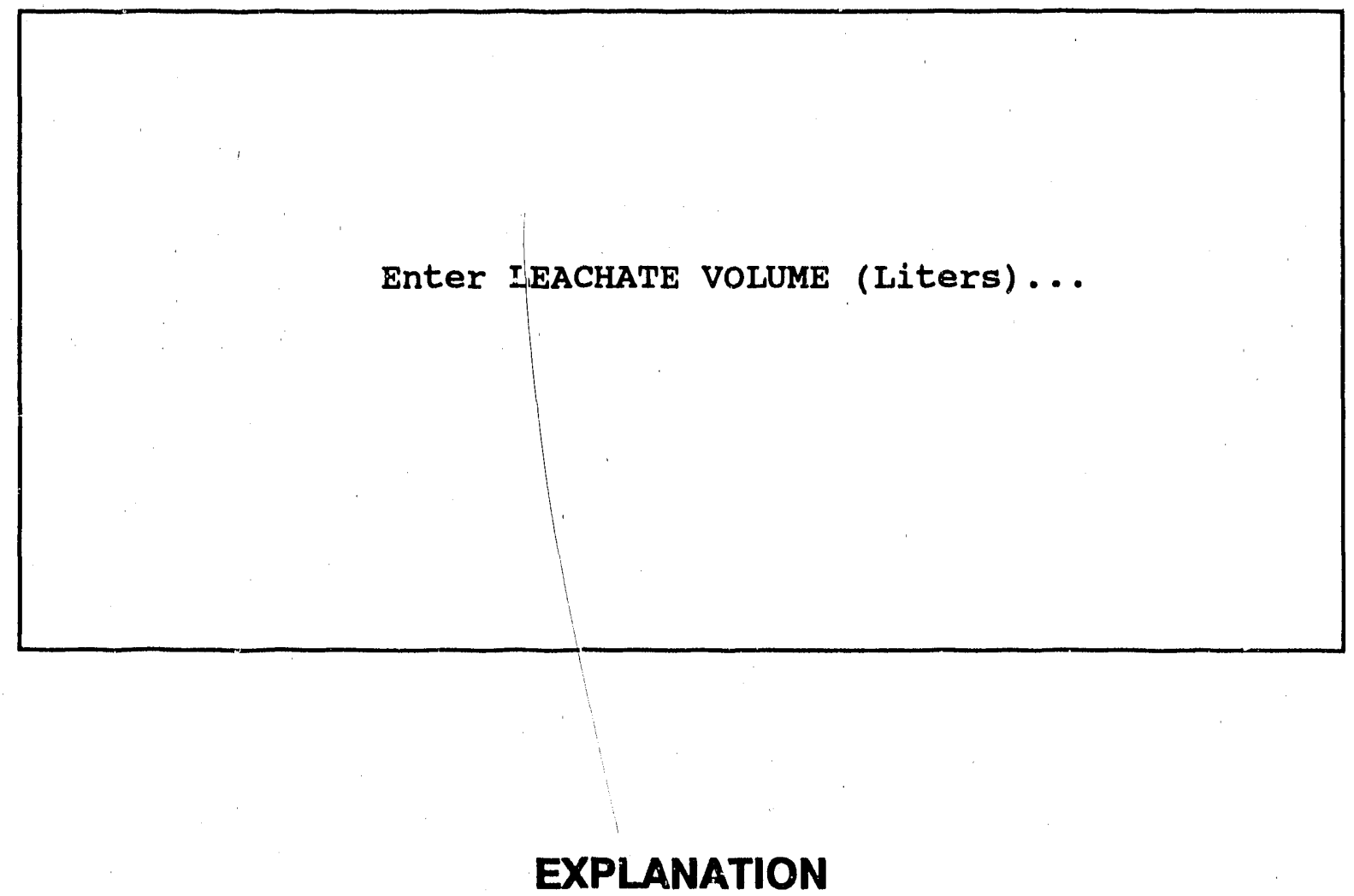

Enter the volume of leachate changed at each sampling increment. The standard test volume is 3.0 liters. 


\section{SCREEN 8A}

$$
\text { Use DEFAULT TIMES (Standard ALT)? [Y]/N }
$$

\section{EXPLANATION}

The times (in days) at which the samples are taken, relative to the start of the test, are input at this point. The default answer $[\mathrm{Y}]$ automatically inputs the standard time intervals used in the accelerated leach test. They are (in days) $0.083,0.208,0.708$, $1.00,1.00,1.00,1.00,1.00,1.00,1.00,1.00,1.00,1.00$.

If you select " $N$," other time intervals (in days) can be input (SEE SCREEN 9A). 


\section{SCREEN 9A}

Enter time interval for sarapling increment 1 ?

\section{EXPLANATION}

If you select " $\mathrm{N}$ " to answer the default times prompt, therl another prompt will appear asking for the time interval (in days) for sampling increment 1. The sampling increments number will advance as answers are provided. This process will stop when the number of increments, input at SCREEN 4A, is reached.

Time intervals are numerical inputs in whole numbers or decimal fractions of days. 


\section{SCREEN 10A}

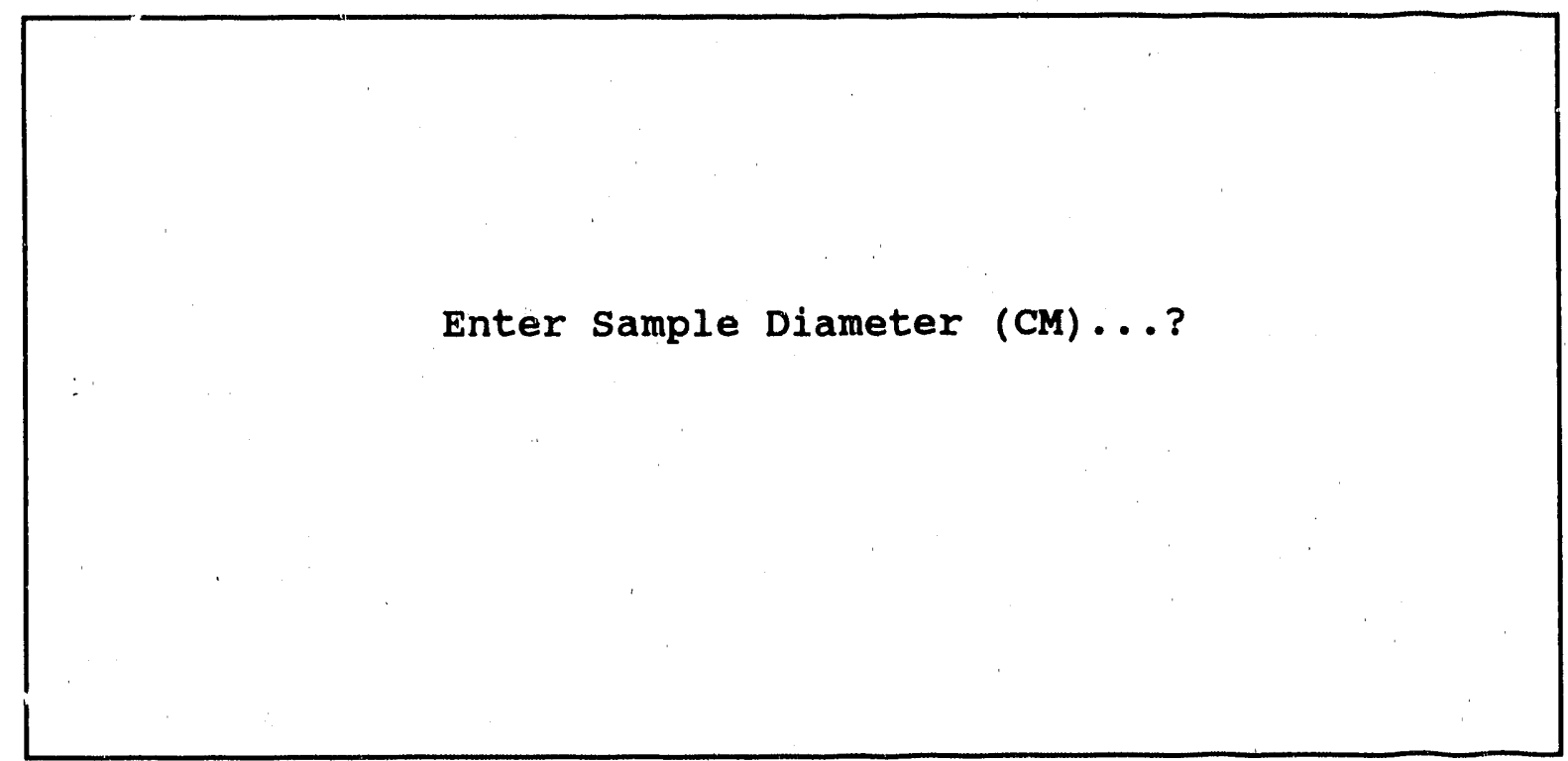

EXPLANATION

Enter the diameter (in centimeters) of the solid sample that was leached. 


\section{SCREEN 11A}

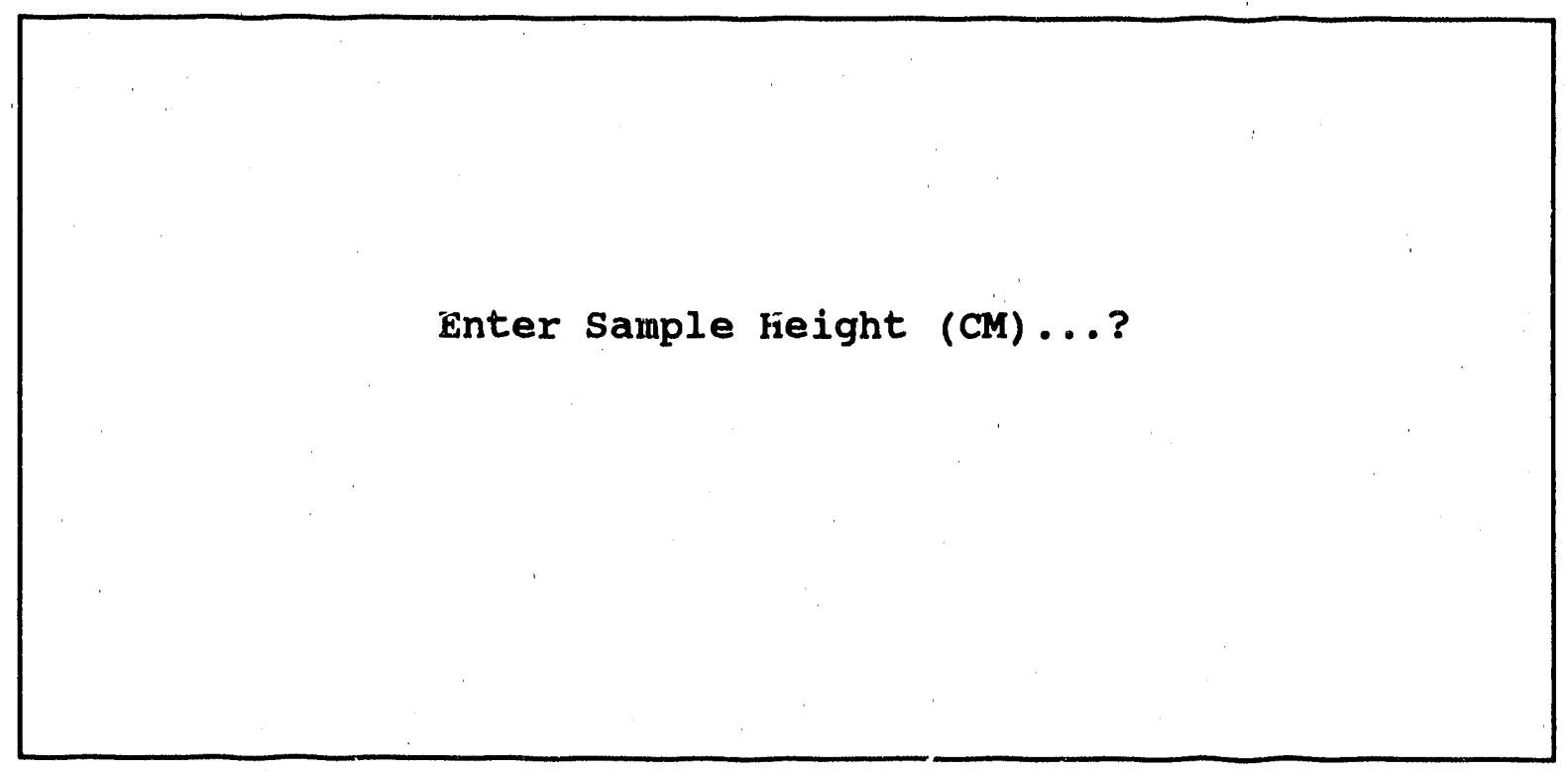

\section{EXPLANATION}

Enter the height (in centimeters) of the solid sample that was leached. 


\section{SCREEN 12A}

Enter Counting Sample size (ml)...?

\section{EXPLANATION}

The volume (in milliliters) of the liquid aliquot that is placed in the detector is entered here. This volume is used to determine the ratio between the size of the sample aliquot and the total volume of leachate. This ratio is then used to multiply the count rate to get counts per minute in the total volume of leachate. 


\section{SCREEN 13A}

Enter Source Term Multiplication Factor...?

\section{EXPLANATION}

The source term multiplication factor is the factor by which an aliquot of the original source solution that was added to the solid samples was diluted to make the counting standard. For example: $3 \mathrm{ml}$ of radioactive tracer were added to a specimen when it was maae, and $1 \mathrm{ml}$ of that solution was diluted 1000 -fold to produce the standard that was counted. The multiplication factor would be 3000 . 
SCREEN 14A

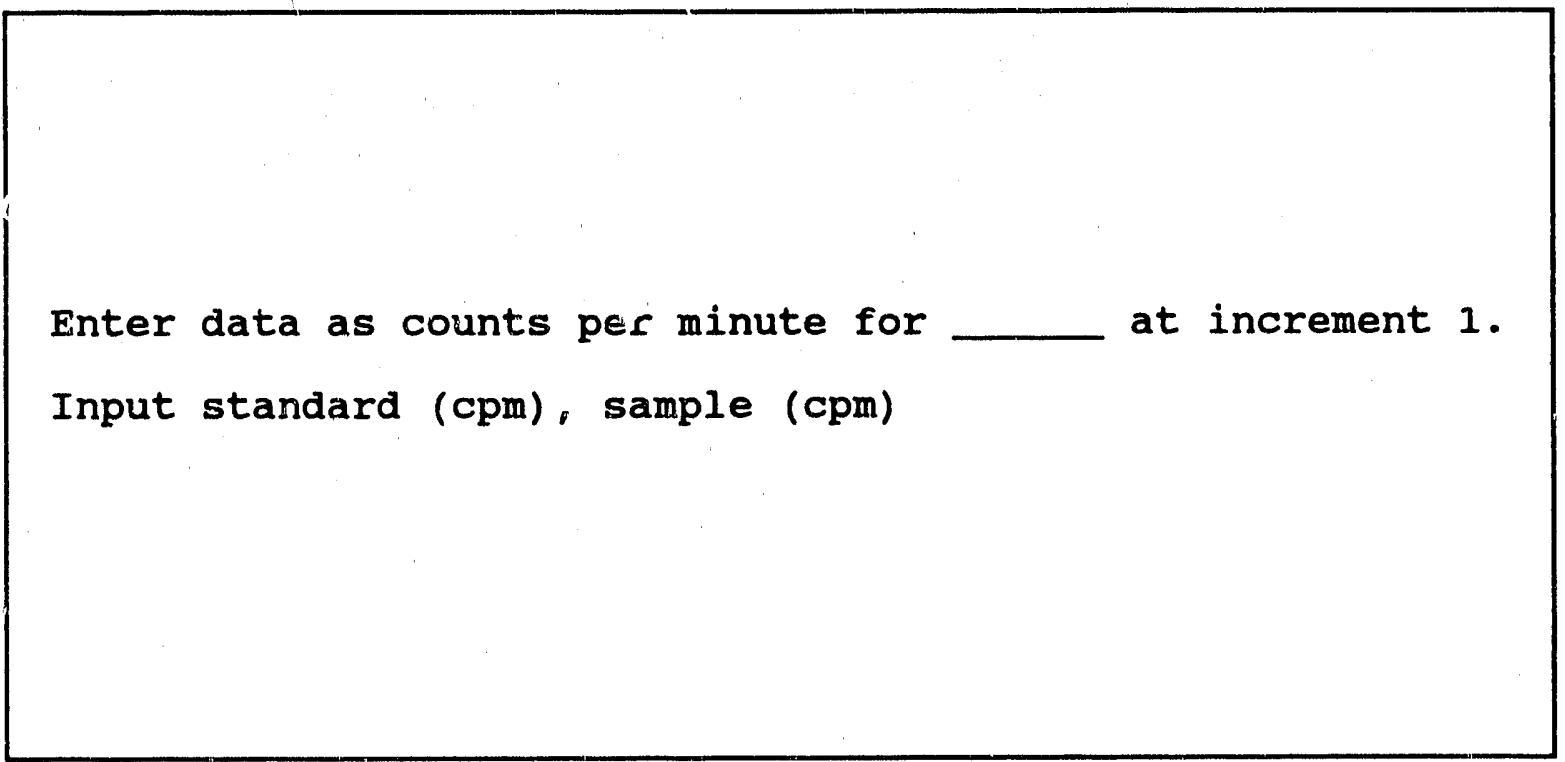

EXPLANATION

The numerical values obtained by radionuclide analysis are entered as counts per rninute (cpm). The first line of the prompt tells what species is bring entered and the sampling increnient. The increment will automatically increase to the value that was input on SCREEN 4A. When the number of increments for the first species has been completed, the species name will automatically switch to the next species.

Input is in the form of cpm and is entered as the counts for the standard and then the counts for the sample. They are separated by a comma. 


\section{SCREEN 15A}

The modeling function will be performed next.

This can be a lengthy process. If any data entry errors were made, you should correct them now to avoid re-running the optimization.

Do you wish to edit the data? $Y /[N]$

\section{EXPLANATION}

With all of the data entered, the program can now perform the calculation and modeling function. Because this can take a significant amount of time, it is best to check the values entered and make any corrections before the calculations begin. If you select " $\mathrm{N}$ " at the prompt, then the program automatically calculates the incremental fraction leached, cumulative fraction leached, diffusion coefficient, and goodness-of-fit values.

If you select "Y" at the prompt, then turn to SCREEN 1G for explanation of the EDIT function. 
SCREEN 16A

Optimizing Parameters for Species \#1

\section{EXPLANATION}

This prompt indicates that calculations are being performed. A rapidly changing number will appear under the line of type as the calculations proceed. This number is increased each time the program completes one set of summations for the model. When the calculations are completed, the program returns to the MAIN MENU. 


\section{SECTION B}

\section{ENTER NEW DATA}

\section{SINGLE SOURCE TERM DATA}




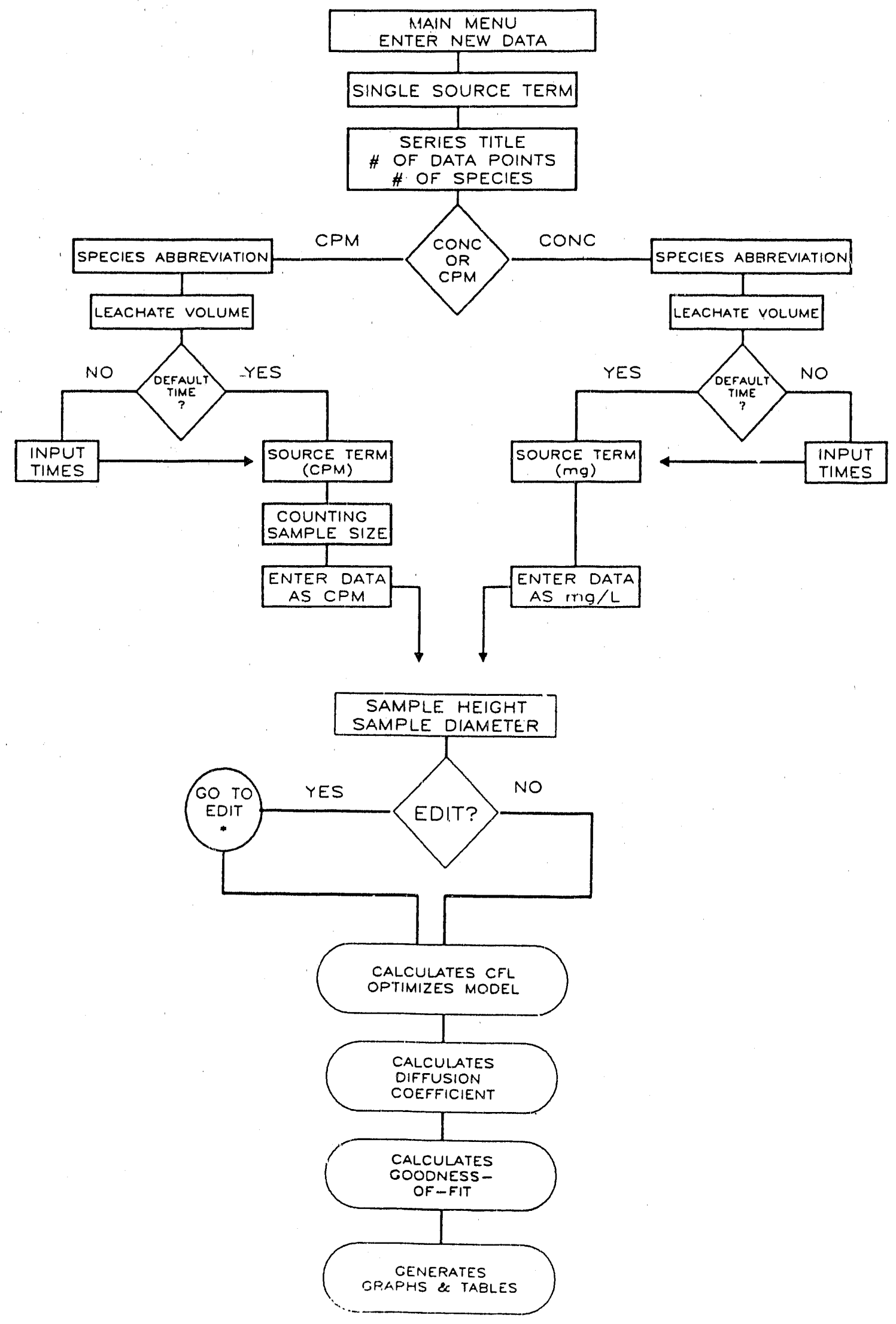

- Fur Edit function see section C 


\section{SCREEN 1B}

Multiple source term data . . . Enter 1 single source term data . . . . Enter 2 To exit and return to main menu .. Enter 3 Enter your choice

\section{EXPLANATION}

If you entered "2," see below.

If you entered "1," turn to SCREEN $1 \mathrm{~A}$.

Single Source Term Data - Some data require only a single value for the source term through the entire experiment. The source term can be in the form of counts per minute (CPM) or as concentration (for stable elements). For some specimens that contain radioactivity, liquid standards may not be available. In this case, the activity in the specimen should be calculated. This value can be input as concentration in the single source term option. 


\section{SCREEN 2B}

$$
\text { Enter SERIES TITLE (max } 40 \text { chars) }
$$

\section{EXPLANATION}

Type in the title that will be used to identify the data set being entered; a maximum of 40 characters is allowed.

This title will be used on tables and graphs that are produced from this data and will help identify data files that will be stored. 


\section{SCREEN 3B}

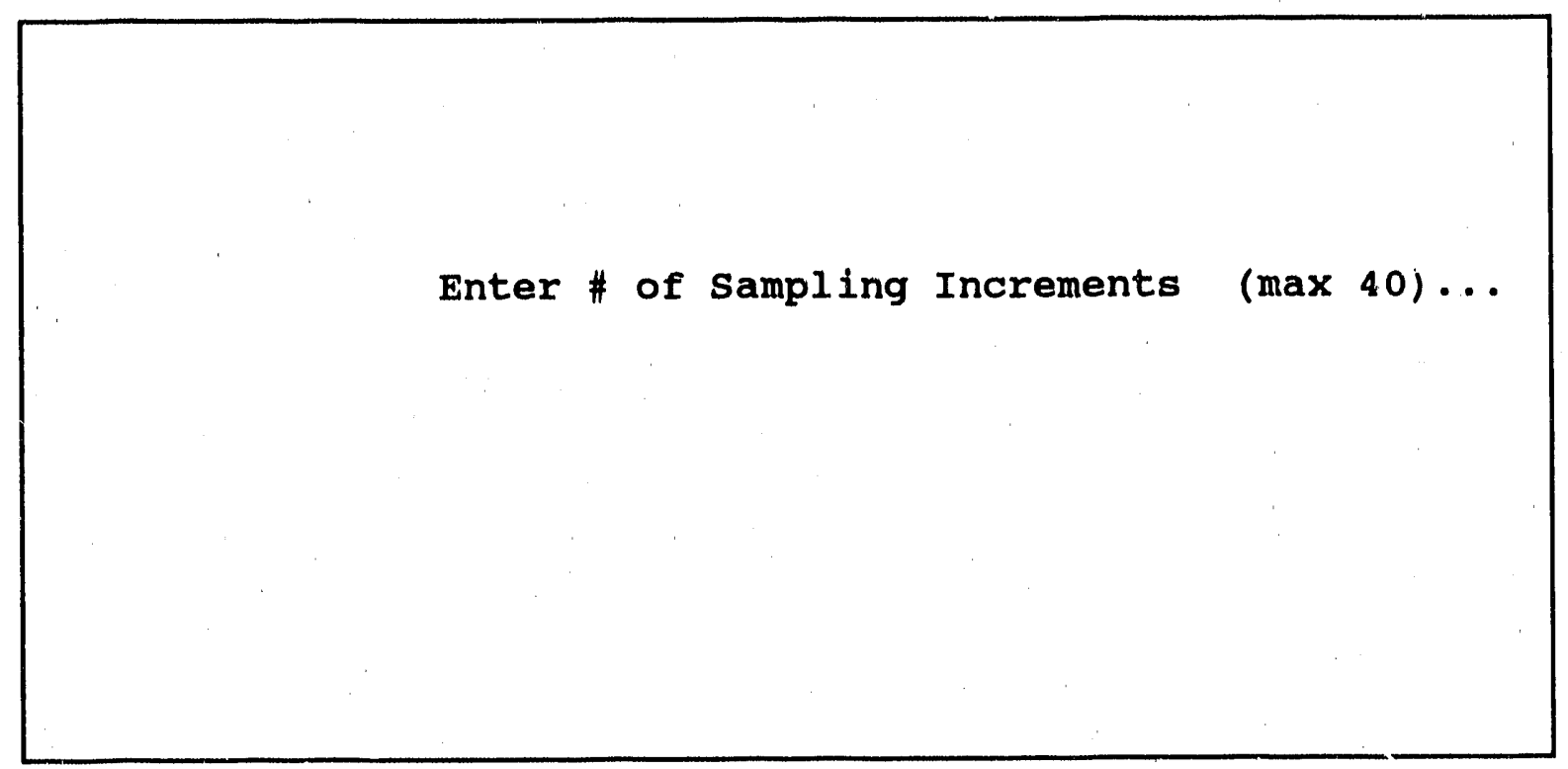

\section{EXPLANATION}

Enter the number of sampling increments in the experiment; the standard test has 13 increments.

The program automatically numbers data as it is input according to the increment. 


\section{SCREEN 4B}

Enter \# of SPECIES (IE Sr, Cs, Co etc) Studied (max 12)...

\section{EXPLANATION}

This input is the number of elements or radionuclides analyzed in each set of leachate samples that need to be addressed by the program. A maximum of twelve (12) species are allowed in each data file. 


\title{
SCREEN 5B
}

\begin{abstract}
Are measurements in concentration or counts/min? CONC/ ( CPM)
\end{abstract}

\section{EXPLANATION}

The program can calculate data that is in the form of concentration (milligrams/liter) or in the form of counts per minute (cpm) for radioactive samples. Calculations are slightly different so the program requires this prompt.

Most screens in SERIES B are the same if either "CONC" or "CPM" are selected. However, SCREENS 10B, 11B and 12B are used with the "CPM" pathway, and SCREENS 13B and 14B are used with the "CONC" pathway. 


\section{SCREEM 6B}

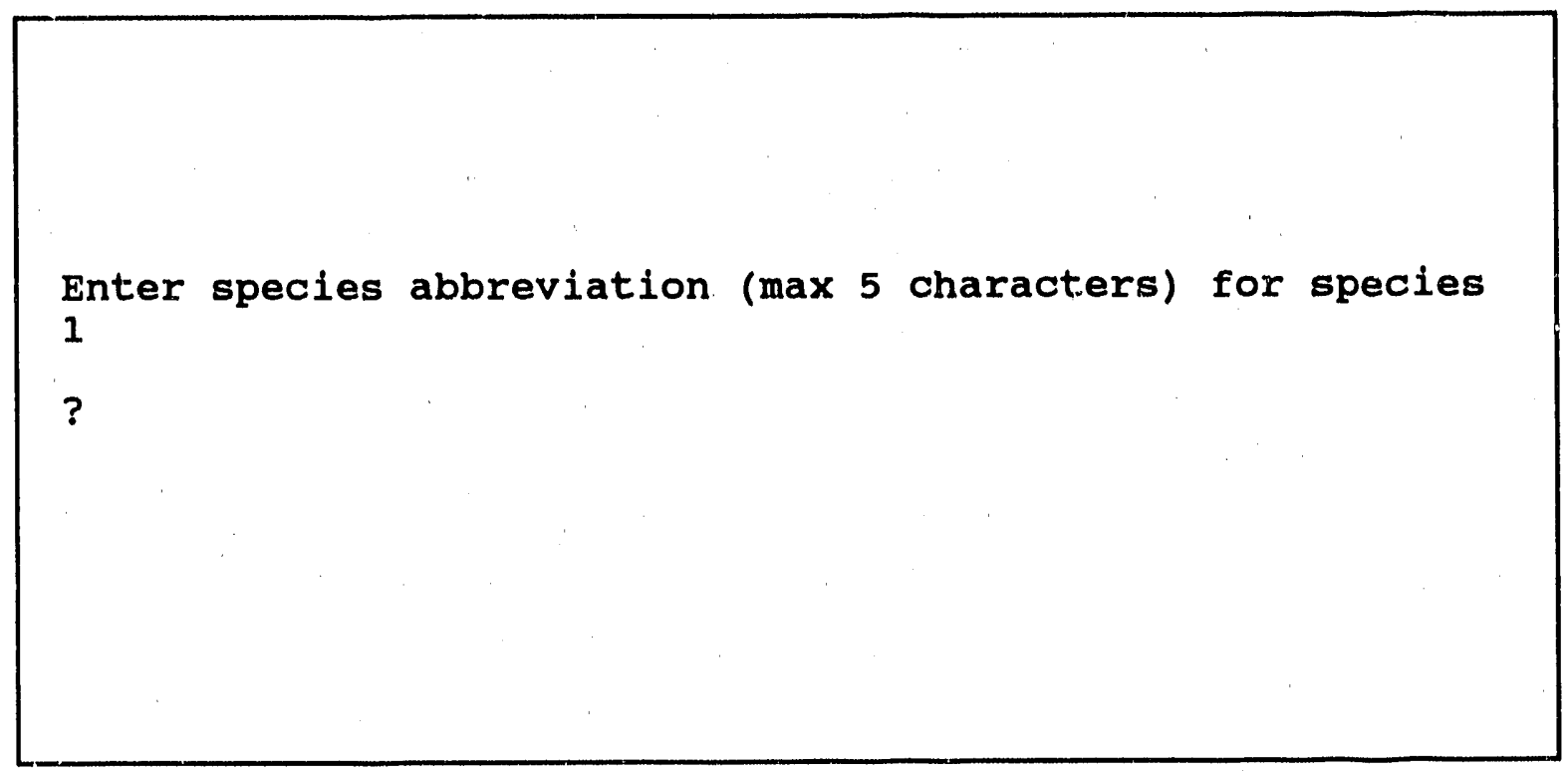

\section{EXPLANATION}

Species abbreviations are letters, numbers, or combinations of the two that are used to identify each of the elements or radionuclides to be considered by the program.

Enter the abbreviation for each of the species as the species number changes in the prompt on the screen. A maximum of 5 characters is allowed. 
SCREEN 7B

Enter leachate volume (liters)...

\section{EXPLANATION}

This is the volume of leachate changed at each sampling increment. The standard test volume is 3.0 liters. 


\title{
SCREEN 8B
}

\author{
Use DEFAULT TIMES (Standard ALT)? [Y]/N
}

\section{EXPLANATION}

The times (in days) at which the samples are taken, relative to the start of the test are input at this point. The default answer [Y] automatically inputs the standard time intervals used in the accelerated leach test. They are (in days) $0.083,0.208,0.708$, $1.00,1.00,1.00,1.00,1.00,1.00,1.00,1.00,1.00,1.00$.

If you select "N," other time intervals (in days) can be input (SEE SCREEN 9B). 


\section{SCREEN 9B}

Enter time interval for sampling increment 1 ?

\section{EXPLANATION}

If you select " $\mathrm{N}$ " to answer the default times prompt, another prompt appears asking for the time interval (in days) for sampling increment 1 . The sampling increment number will advance as answers are provided. This process will stop when the number of intervals, input at SCREEN 4B, is reached.

The time intervals are numerical inputs in whole numbers or decimal fractions of days. 


\section{SCREEN 10B}

Enter SOURCE TERM (CPM) for species___...?

\section{EXPLANATION}

Use this page only if you selected "CPM" at SCREEN 5B.

If you selected "CONC," go to SCREEN 13B.

The source term is the amount of activity (in counts-per-minute for each radionuclide) that was originally contained in the solid sample at the start of the leach test. The source term for each species is entered following the prompt for that species. 


\section{SCREEN 11B}

$$
\text { Enter counting sample size (ml) } \ldots \text { ? }
$$

\section{EXPLANATION}

Use this page only if you selected "CPM" at SCREEN 5B.

The volume (in milliliters) of the liquid aliquot that was analyzed is entered here. This volume is used to determine the ratio between the size of the sample aliquot and the total volume of leachate. This ratio is then used to multiply the count rate to get counts per minute in the total volume of leachate. 


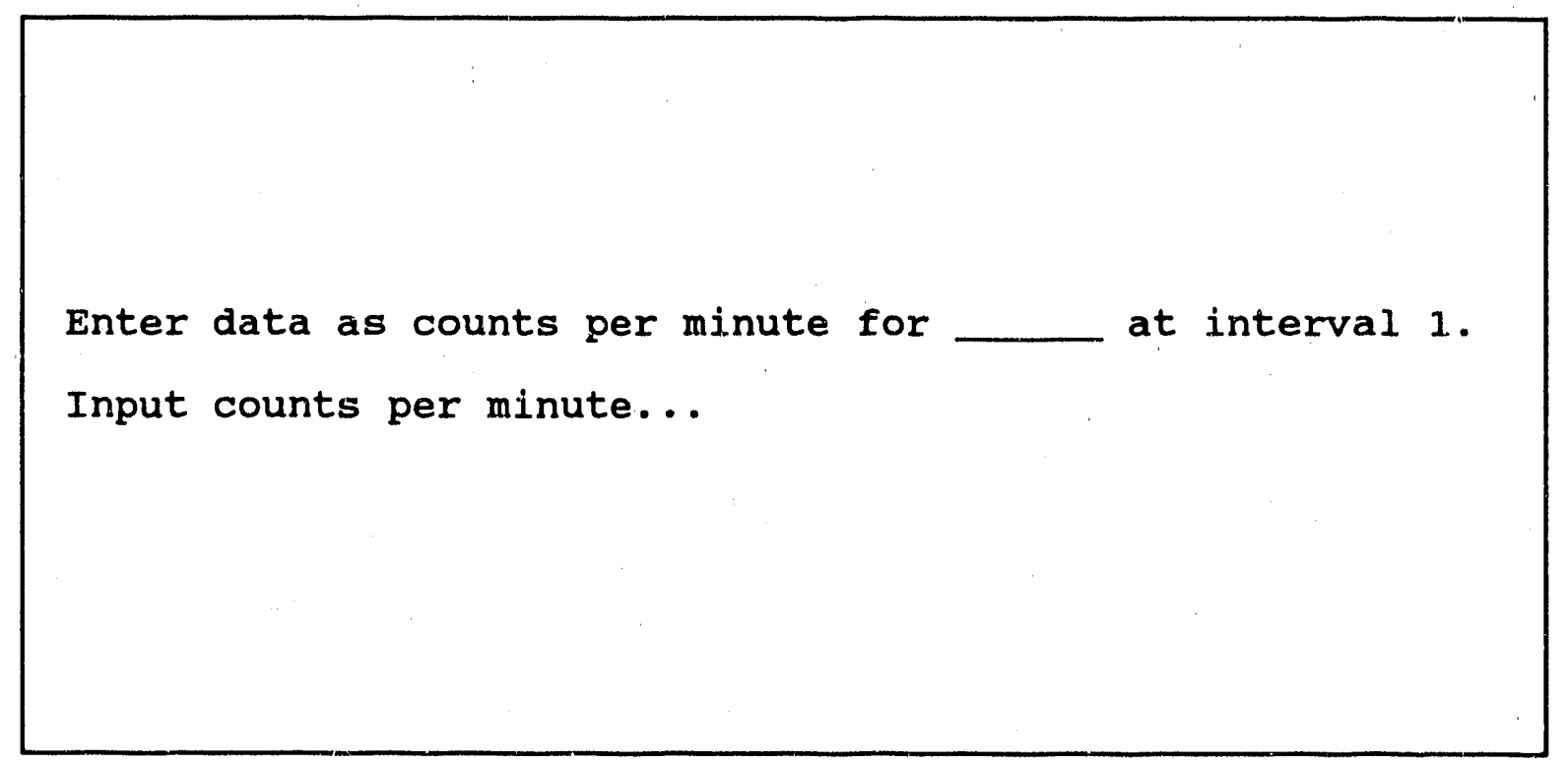

\section{EXPLANATION}

Use this page only if you selected "CPM" at SCREEN 5B.

The numerical values obtained by radionuclide analysis are entered as counts per minute (cpm). The first line of the prompt tells what species is being entered and the interval. The interval will automatically increase to the value input on SCREEN 4A. When the number of intervals for the first species has been completed, the species name will automatically switch to the next species. 


\section{SCREEN 13B}

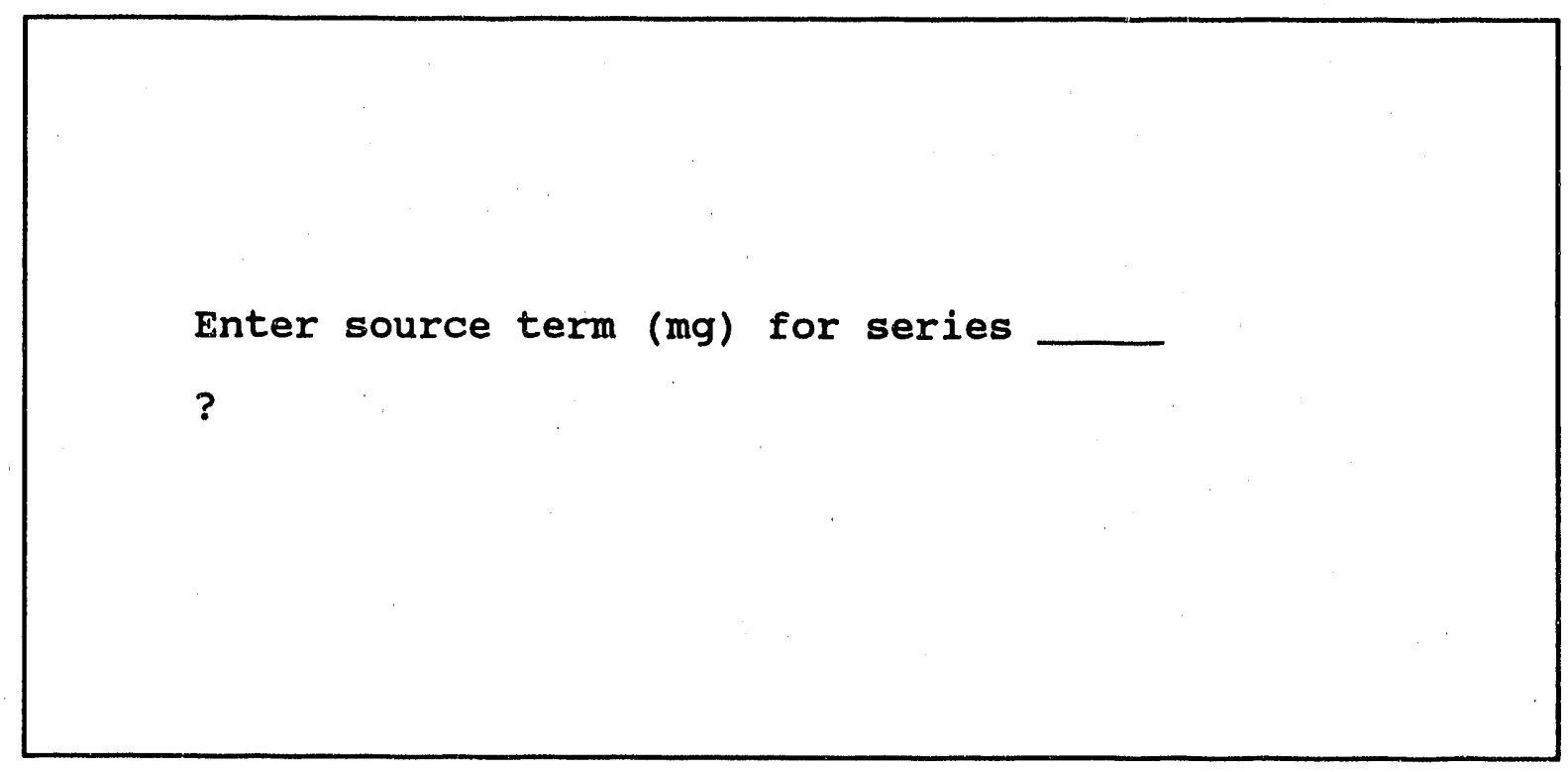

EXPLANATION

Use this page only if you selected "CONC" at SCREEN 5B.

The source term is the amount of a species (in r.iilligrams for each species) that was originally contained in the solid sample at the start of the leach test. Enter the source term for each species after the prompt for that species. 


\section{SCREEN 14B}

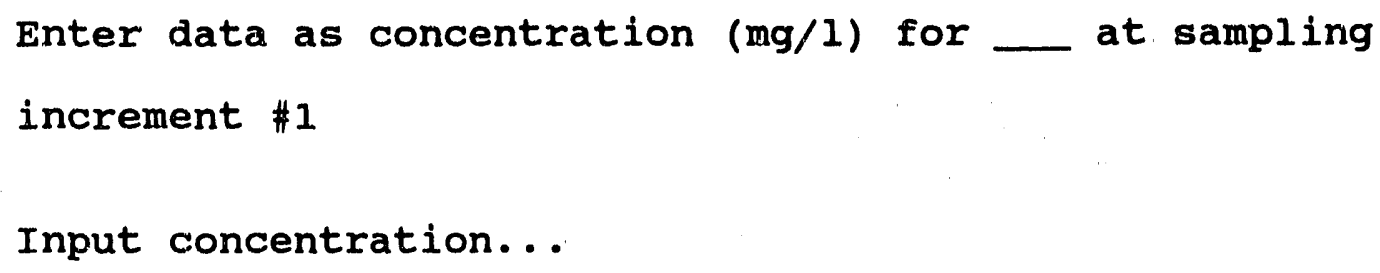

Use this page only if you selected "CONC" at SCREEN 5B.

Concentration in the leachate as milligrams/liter (ppm) are entered for each species for the sampling increment indicated in the prompt. The sampling increment will increase automatically to the maximum increment, input at SCREEN 3B. The species indicated in the prompt will also advance automatically. 
SCREEN 15B

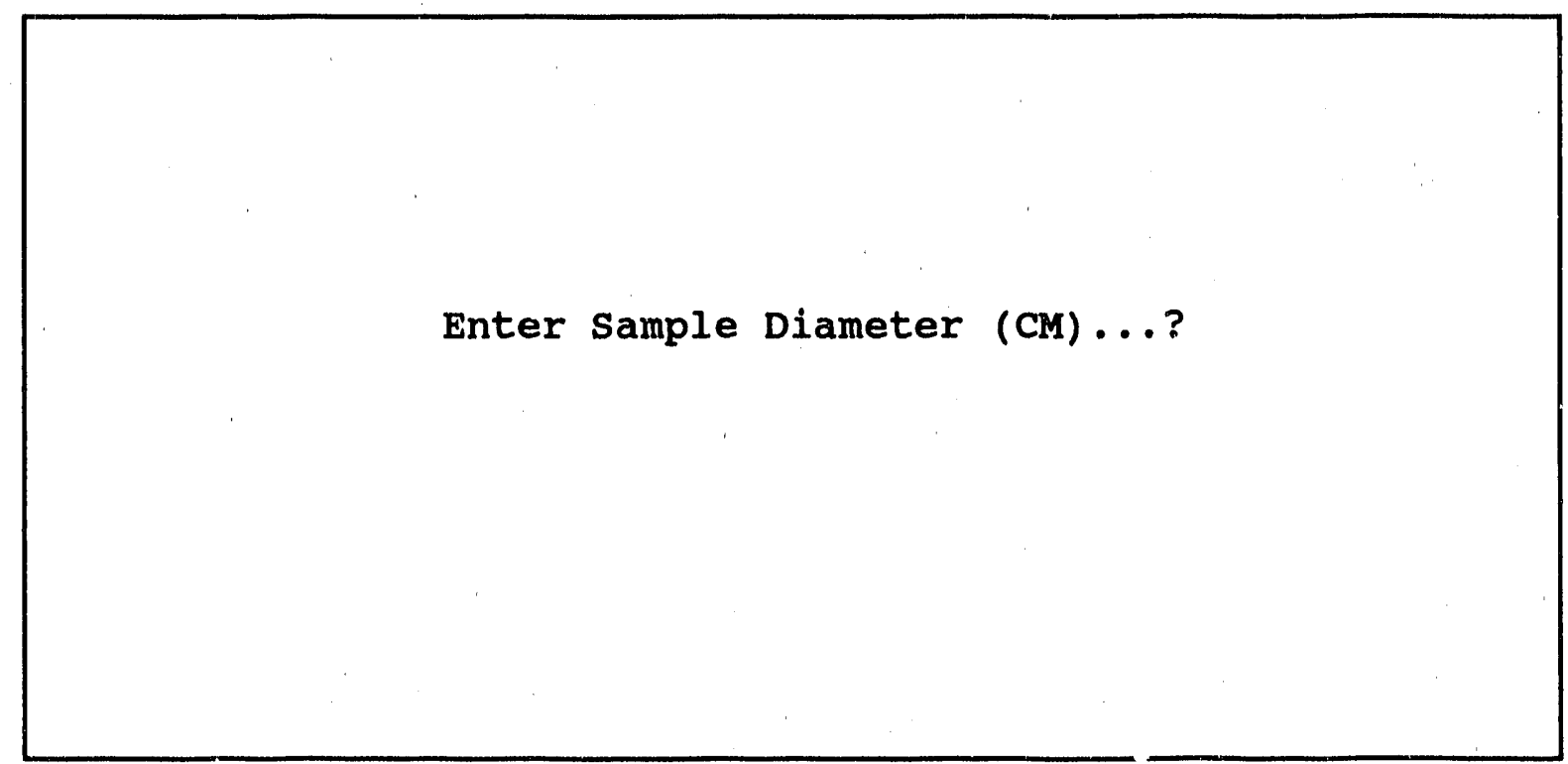

\section{EXPLANATION}

Enter the diameter (in centimeters) of the solid sample that was leached. 


\section{SCREEN 16B}

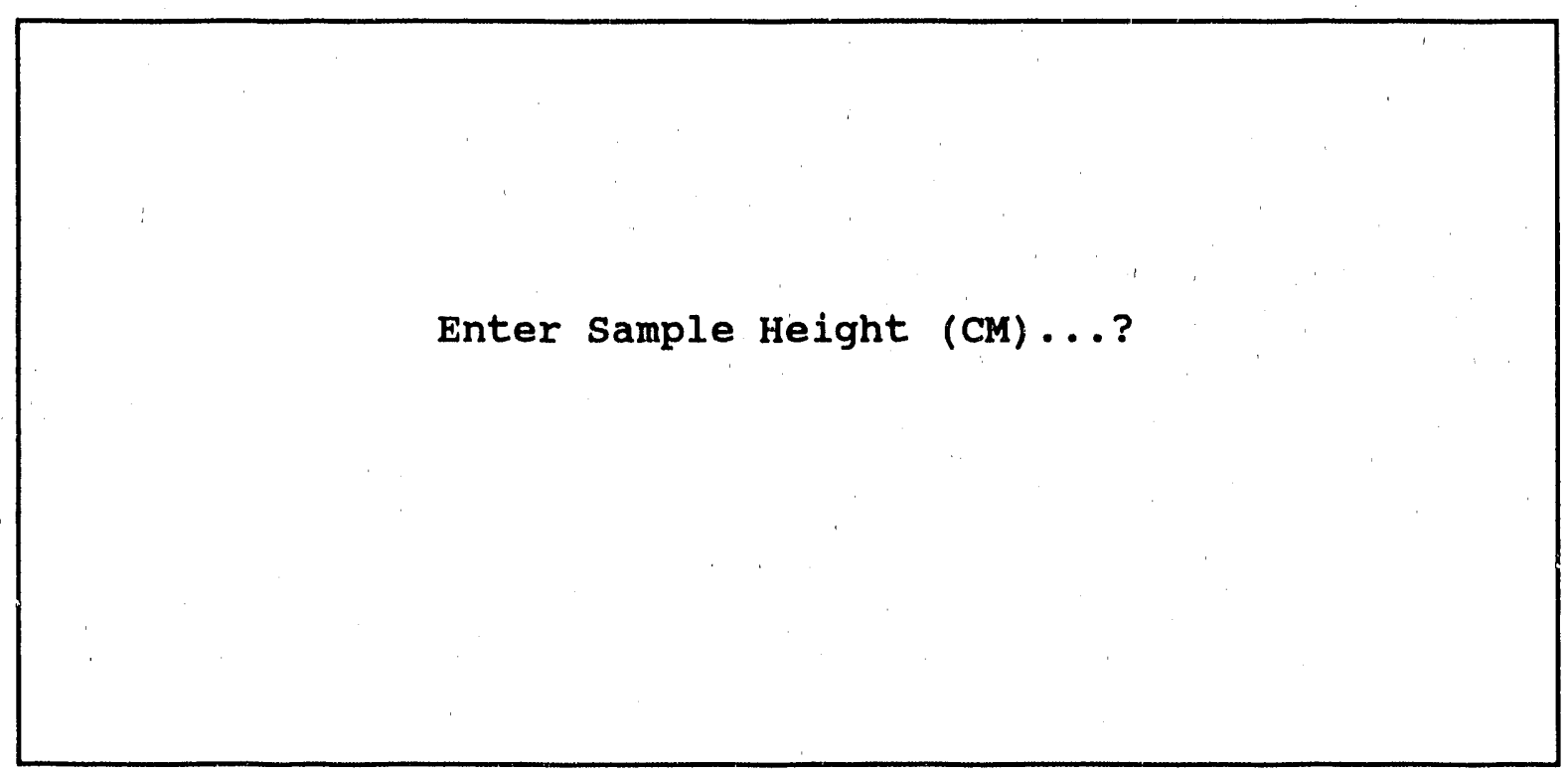

\section{EXPLANATION}

Enter the height (in centimeters) of the solid sample that was leached. 


\section{SCREEN 17B}

The modeling cunction will be performed next.

This can be a lengthy process. If any data entry errors were made, you should correct them now tc avoid re-running the optimization.

Do you wish to edit the data? $Y /[N]$

\section{EXPLANATION}

With all of the data entered, the program can now perform the calculation and modeling function. Because this function can take a significant amount of time, it is best to check the values entered and make any corrections before the calculations begin. If you selected " $N$ " at the prompt, then the program automatically calculates the incremental fraction leached, cumulative fraction leached, diffusion coefficient, and goodness-of-fit values.

If you select "Y" at the prompt, then turn to SCREEN 1G for an explanation of the EDIT function. 


\section{SCREEN 18B}

Optimizing model parameters for species \#1

\section{EXPLANATION}

This prompt indicates that calculations are being performed. A rapidly changing number will appear under the line of type as the calculations proceed. This number is increased each time the program completes one set of summations for the model. When the calculations are completed, the program returns to the MAIN MENU. 


\title{
SECTION C
}

\section{ENTER DATA AS}

\author{
CFL vs TIME
}




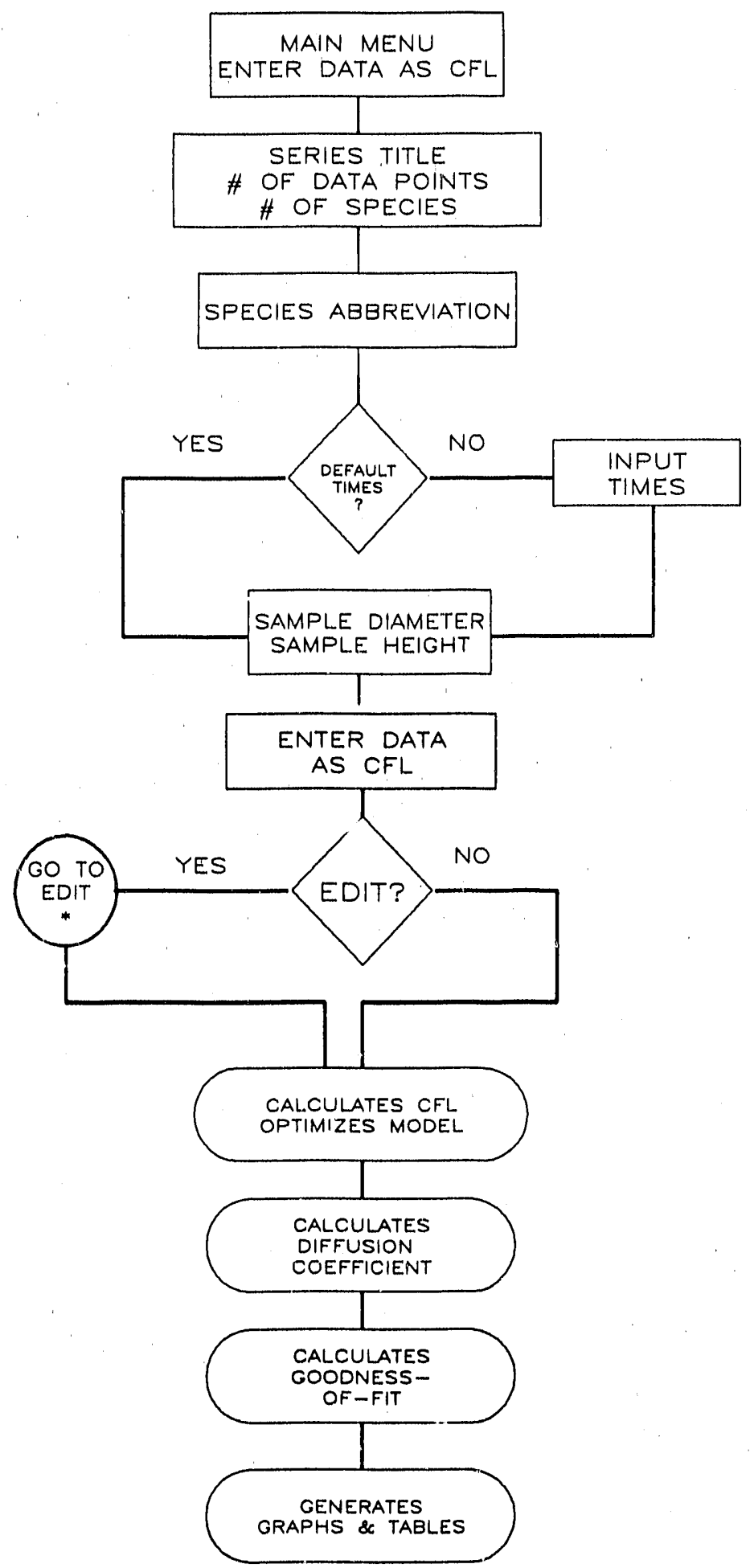

* Fcr Edit function see section $G$ 


\section{SCREEN 1C}

Use the cursor keys to highlight your choice, then press enter to choose it.

Enter new (raw) data

Enter data as CFL Vs Tim?

Retrieve a data set

store a data set

Print a final data set

Edit data

To add data to a file

Plot data

Plot comparison

Exit program

Extend modeled curve

\section{Developed for \\ DOE National Low-Level Waste Management Program by \\ Brookhaven National Laboratory \\ Low-Level Waste Research Group \\ Upton, N.Y. 11973}

Press Fl for help and definitions

\section{EXPLANATION}

Enter Data As CFL vs Time - Select this function if data is already available as cumulative fraction leached, which allows easier input than with the "new data" function. 


\section{SCREEN 2C}

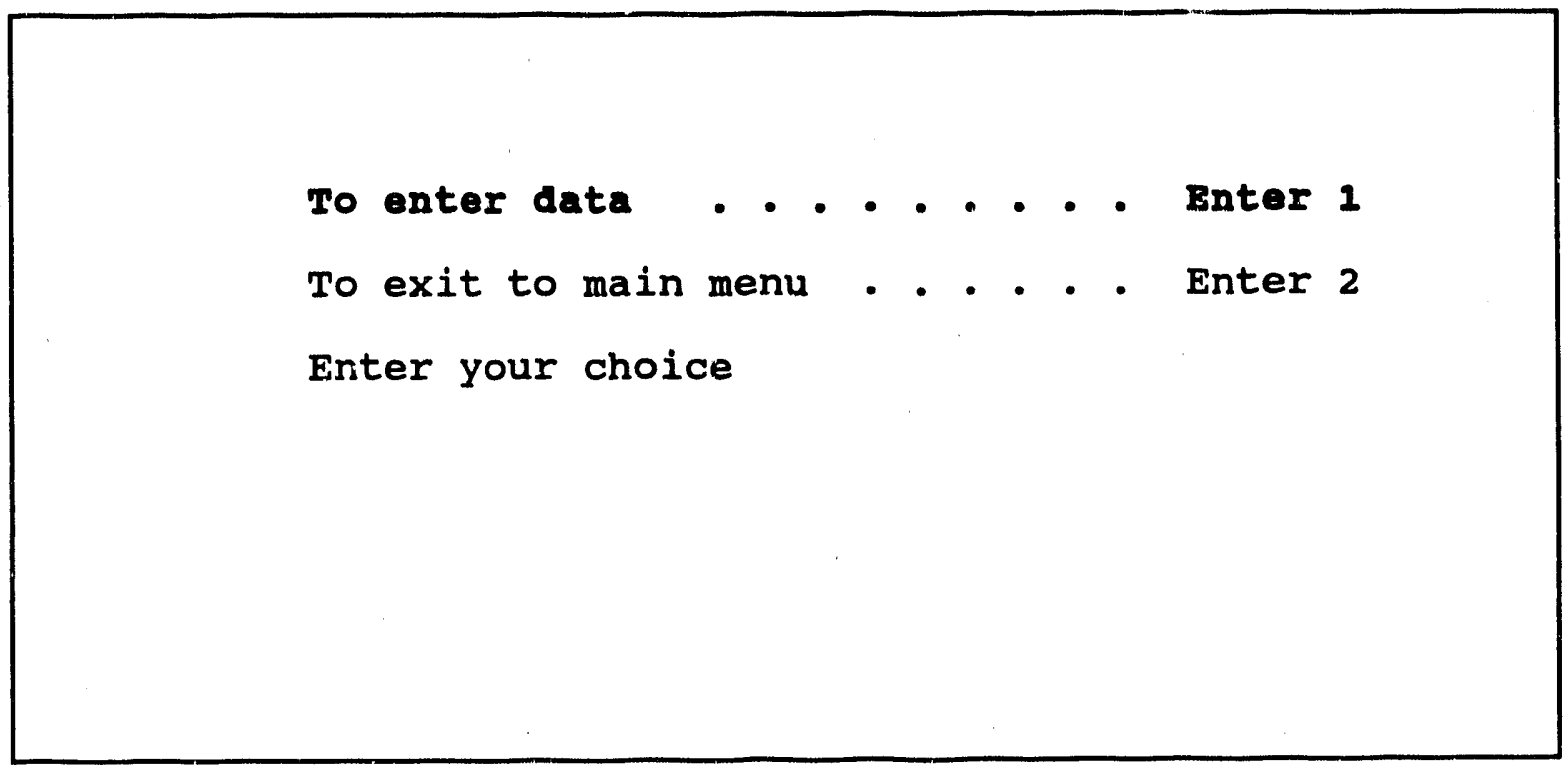

\section{EXPLANATION}

Select " 1 " to enter the pathway, allowing input of data in the form of cumulative fraction leached. Select " 2 " to return to the MAIN MENU. 


\section{SCREEN 3C}

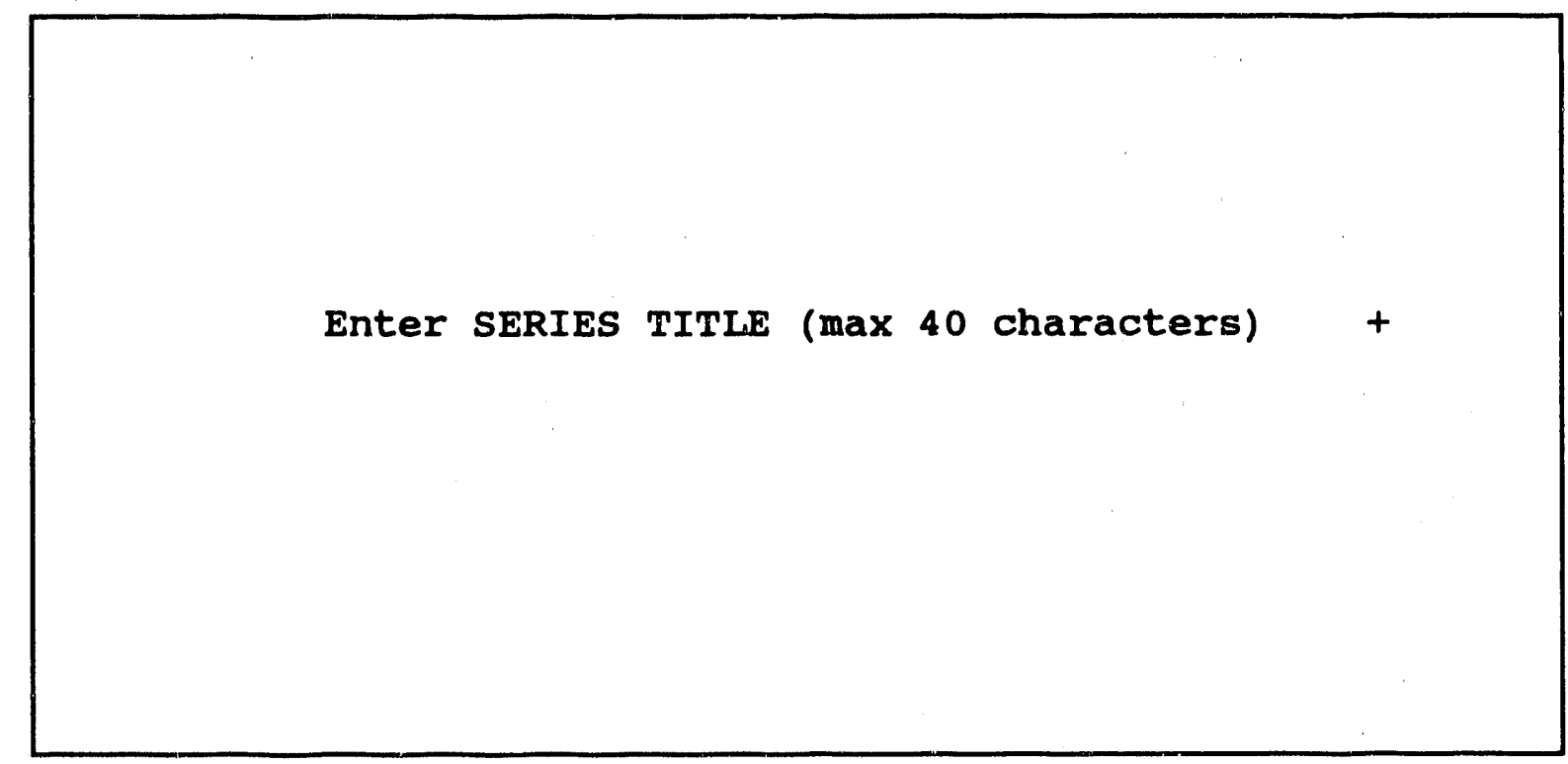

\section{EXPLANATION}

Type in the title that will be used to identify the data set being entered. A maximum of 40 characters is allowed; you cannot type beyond the plus sign.

This title will be used on tables and graphs that are produced from this data and will help identify data files that will be stored. 


\section{SCREEN 4C}

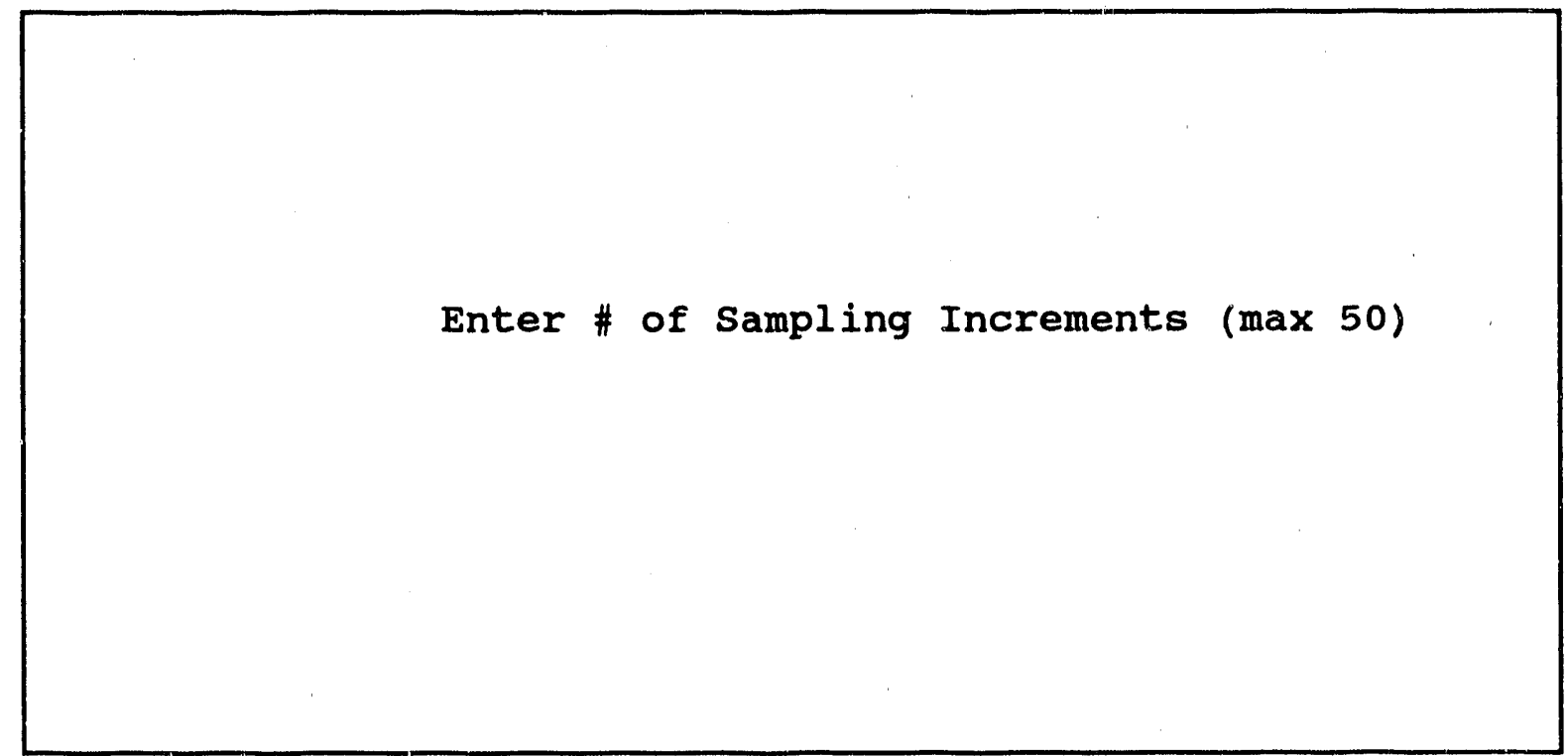

\section{EXPLANATION}

Enter the number of sampling increments in the experiment; the standard test has 13 increments.

The program automatically numbers data as it is input according to the increments. 


$$
\text { Enter \# of SPECIES (IE Sr, Cs, Co etc) (max 12) }
$$

\section{EXPLANATION}

This input is the number of elements or radionuclides analyses in each set of leachate samples that need to be addressed by the program. A maximum of twelve species is allowed in each data file. 
SCREEN 6C

Enter SPECIES ABBREVIATION (max 5 characters) for species 1

\section{EXPLANATION}

Species abbreviations are letters, numbers, or combinations of the two that are used to identify each of the elements or radionuclides to be considered by the program.

Enter the abbreviation for each of the species as the species number changes in the prompt on the screen. A maximum of 5 characters is allowed. 


\section{SCREEN 7C}

Use DEFAULT TIMES (Standard ALT)? [Y]/N

\section{EXPLANATION}

The times (in days) at which the samples are taken, relative to the start of the test, are input at this point. The default answer $[Y]$ automatically inputs the standard time intervals used in the accelerated leach test. They are (in days) $0.083,0.208,0.708$, $1.00,1.00,1.00,1.00,1.00,1.00,1.00,1.00,1.00,1.00$.

If you select "N," other time intervals (in days) can be input (SEE SCREEN 9C). 


\section{SCREEN 8C}

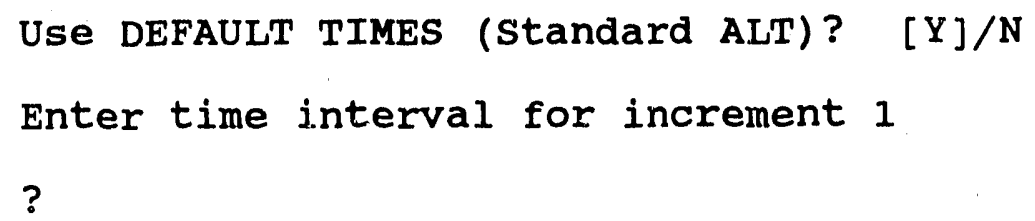

\section{EXPLANATION}

If you select " $\mathrm{N}$ " to answer the default times prompt, another prompt appears asking for the time interval (in days) for increment 1. The increment number will advance as answers are provided. This process will stop when the number of increments, input at SCREEN 4, is reached.

The tirne intervals are numerical inputs in whole numbers or decimal fractions of days. 


\section{SCREEN 9C}

Enter Sample Diameter (CM)...?

\section{EXPLANATION}

Enter the diameter (in centimeters) of the solid sample that was leached. 


\section{SCREEN 10C}

$$
\text { Enter Sample Height (CM) ...? }
$$

\section{EXPLANATION}

Enter the height (in centimeters) of the solid sample that was leached. 


\section{SCREEN 11C}

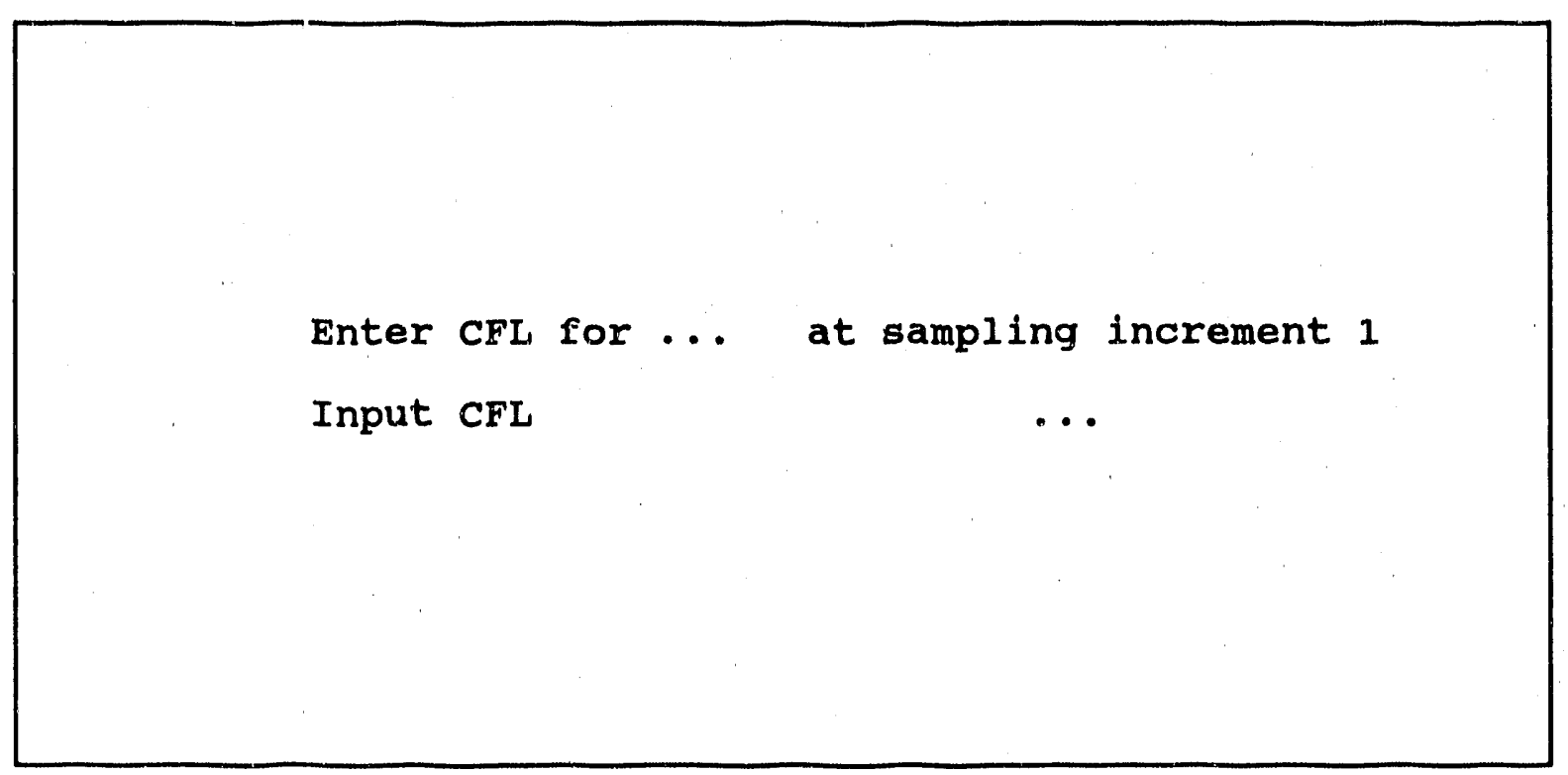

\section{EXPLANATION}

Enter the cumulative fraction leached (CFL) as the decimal value for the species and sampling increment shown on the prompt. The increment will automatically increase to the value input at SCREEN 4C. When the number of sampling increments for the first species has been completed, the species name will automatically switch to the next species. 
The modeling function will be performed next.

This can be a lengthy process. If any data entry errors were made, you should correct them now to avoid re-running the optimization.

Do you wish to edit the data? $\mathrm{Y} /[\mathrm{N}]$

\section{EXPLANATION}

With all of the data entered, the program can now perform calculation and modeling function. Because this function can take a significant amount of time, it is best to check the values entered and make any corrections before the calculations begin. If you answered " $\mathrm{N}$ " at the prompt, then the program automatically calculates incremental fraction leached, cumulative fraction leached, diffusion coefficient, and goodness-of-fit values.

If you answered " $Y$ " at the prompt, then turn to SCREEN 1G for explanation of the EDIT function. 


\section{SCREEN 13C}

$$
\text { optimizing model parameters for species \#1 }
$$

\section{EXPLANATION}

This prompt indicates that calculations are being performed. A rapidly changing number will appear under the line of type as the calculations proceed. This number is increased each time the program completes one set of surnmations for the model. When the calculatiuns are completed, the program returns to the MAIN MENU. 


\section{SECTION D}

\section{RETRIEVE A DATA SET}




\section{SCREEN 1D}

Use the cursor keys to highlight your choice, then press enter to choose it.
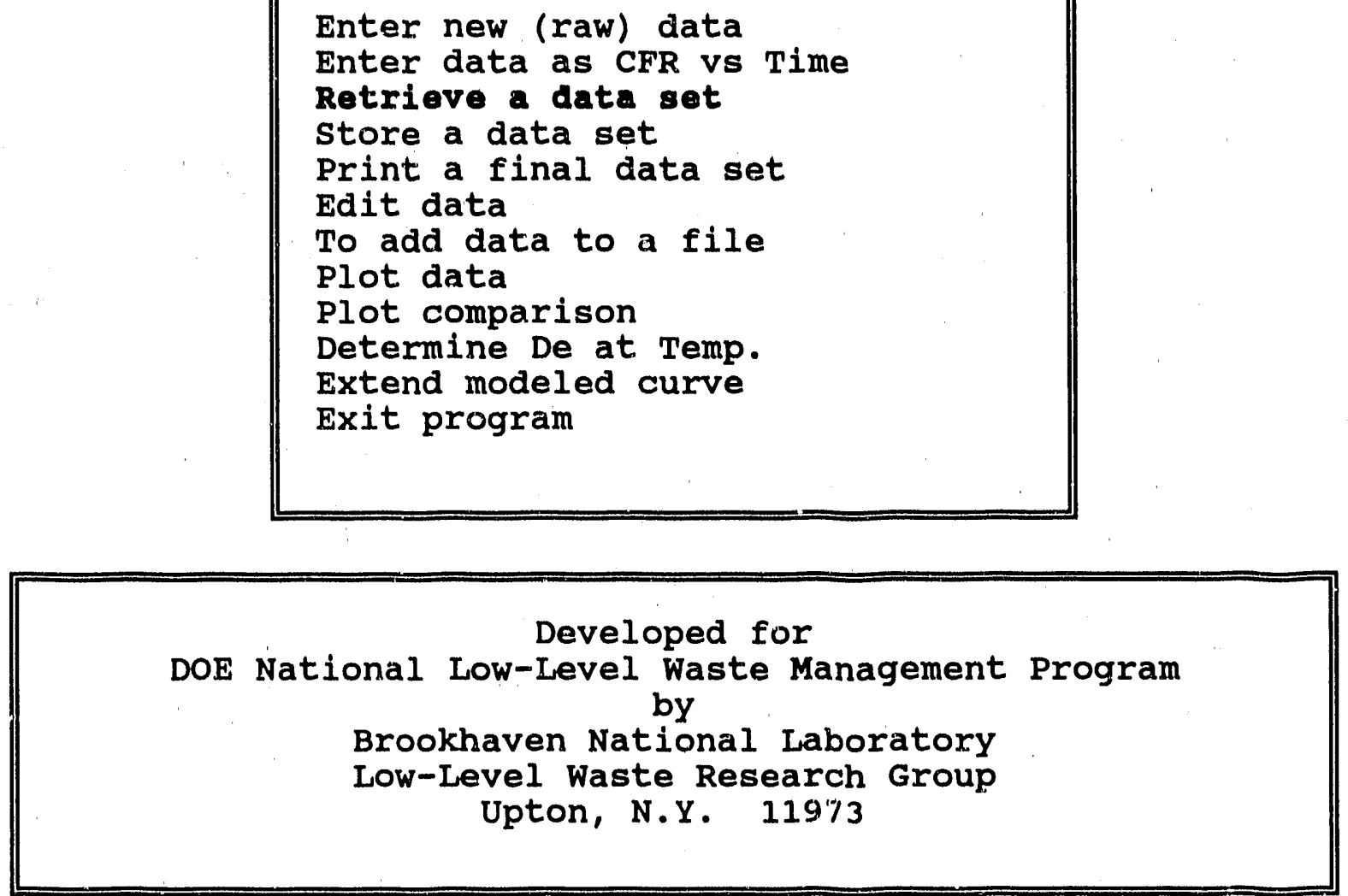

Press F1 for help and definitions

\section{EXPLANATION}

Retrieve A Data Set is selected in order to take a data set from a floppy disk or a hard disk and bring it into the "ALT" program for use. 


\section{SCREEN 2D}

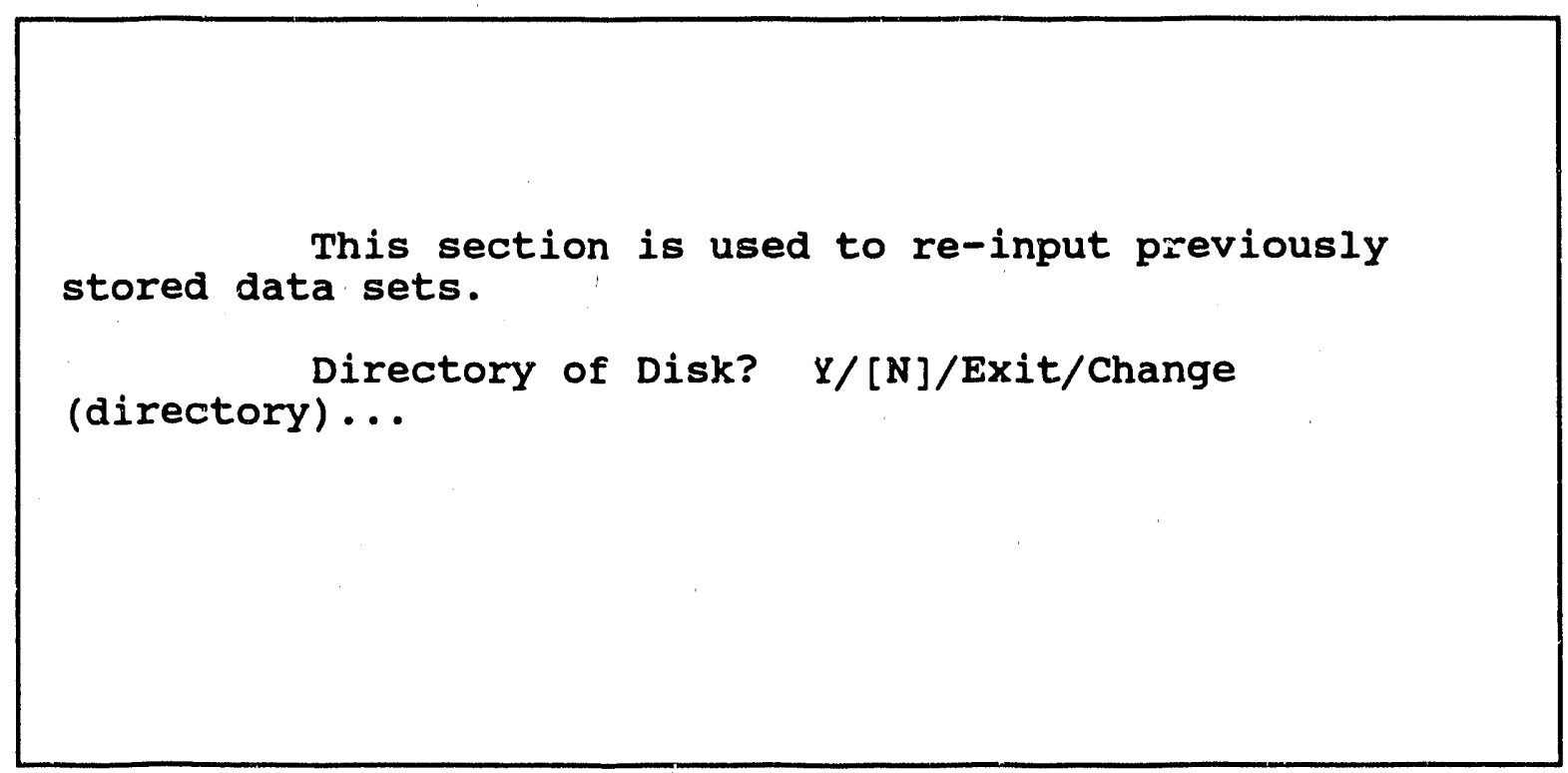

\section{EXPLANATION}

If you need a directory of the disk, type "Y." A listing of the files on the disk will then appear on the screen.

If you do not need a directory of the disk, press "Enter" and turn to SCREEN 3D.

If you need a change of directory, type " $C$ " and turn to SCREEN 4D. 


\section{SCREEN 3D}

Enter file name ( $\max 8$ characters)...

\section{EXPLANATION}

Enter the name of the file to be retrieved; a maximum of 8 characters is allowed. Do not specify the file extension; the program automatically searches for the .ALT extension. When the file is retrieved, the user is returned to the MAIN MEiNU.

If that file name cannot be found on the disk, an error message appears saying: "File not Found," and the user is returned to SCREEN 2D. 


\section{SCREEN 4D}

Enter new dxive: \pathway \

\section{EXPLANATION}

This prompt allows the user to change the disk drive from which data is retrieved. It also allows the pathway on that drive to be specified.

To change the directory, type the drive specifier $(A, B, C)$, followed by ": $\backslash$ " followed by pathway $\backslash$. A backslash $(\backslash$ ) is required at the ena of the pathway. The program will then return to SCREEN $2 D$. 


\section{SECTION E}

\section{STORE A DATA SET}




\section{SCREEN 1E}

Use the cursor keys to highlight your choice, then press enter to choose it.

Enter new (raw) data

Enter data as CFR vs Time

Retrieve a data set

store a data set

Print a final data set

Edit data

To add data to a file

Plot data

Plot comparison

Determine De at Temp.

Extend modeled curve

Exit program

Developed for

DOE National Low-Level Waste Management Program

by

Brookhaven National Laboratory

Low-Level Waste Research Group

Upton, N.Y. 11973

Press F1 for help and definitions

\section{EXPLANATION}

Store a data set is selected to create a file and store it on either a floppy disk or a hard disk. 


\section{SCREEN 2E}

$$
\text { Save as .ALT or .WKS (LOTUS compatible) file [A]/W... }
$$

\section{EXPLANATION}

The program can save data files in two formats; one which is compatible with the "ALT" program itself (.ALT), and the other (.WKS) which is compatible with LOTUS 1-2-

3 (and therefore, a variety of graphics packages). At this prompt the user can select the format in which the data will be saved.

The program cannot use.WKS type files. If it is intended to rerun the data, it should be saved as an .ALT file. We recommend that files be saved in the ALT format and then resaved as.WKS when the need arises to use LOTUS 1-2-3, or to produce publication quality graphics. 


\section{SCREEN 3E}

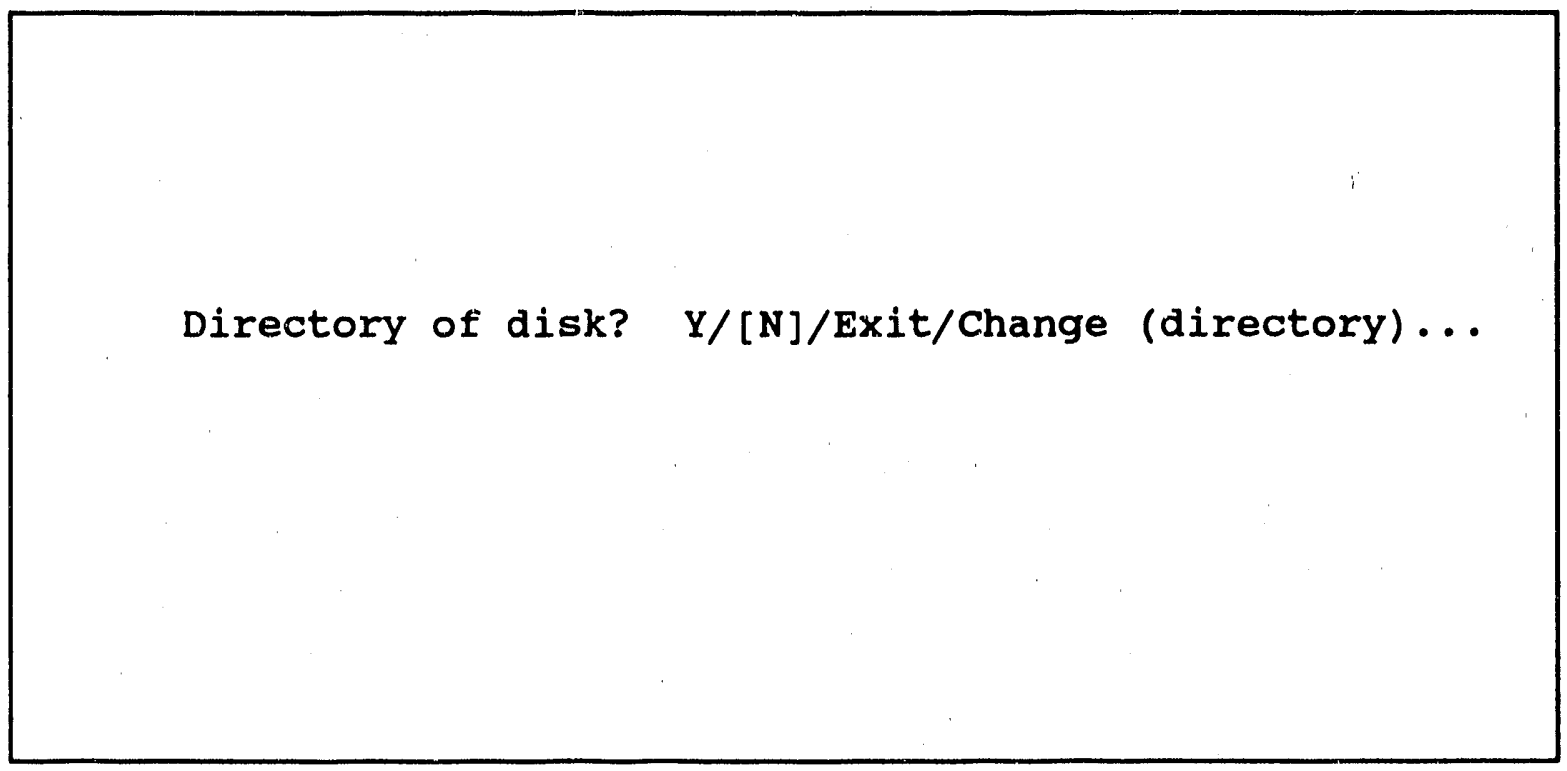

\section{EXPLANATION}

If you need a directory of the disk, type "Y." A listing of the files on the disk will then appear on the screen.

If you do not need a directory of the disk, press "Enter" and turn to SCREEN 3E.

If you need a change of directory, type "C" and turn to SCREEN 4E. 


\section{SCREEN 4E}

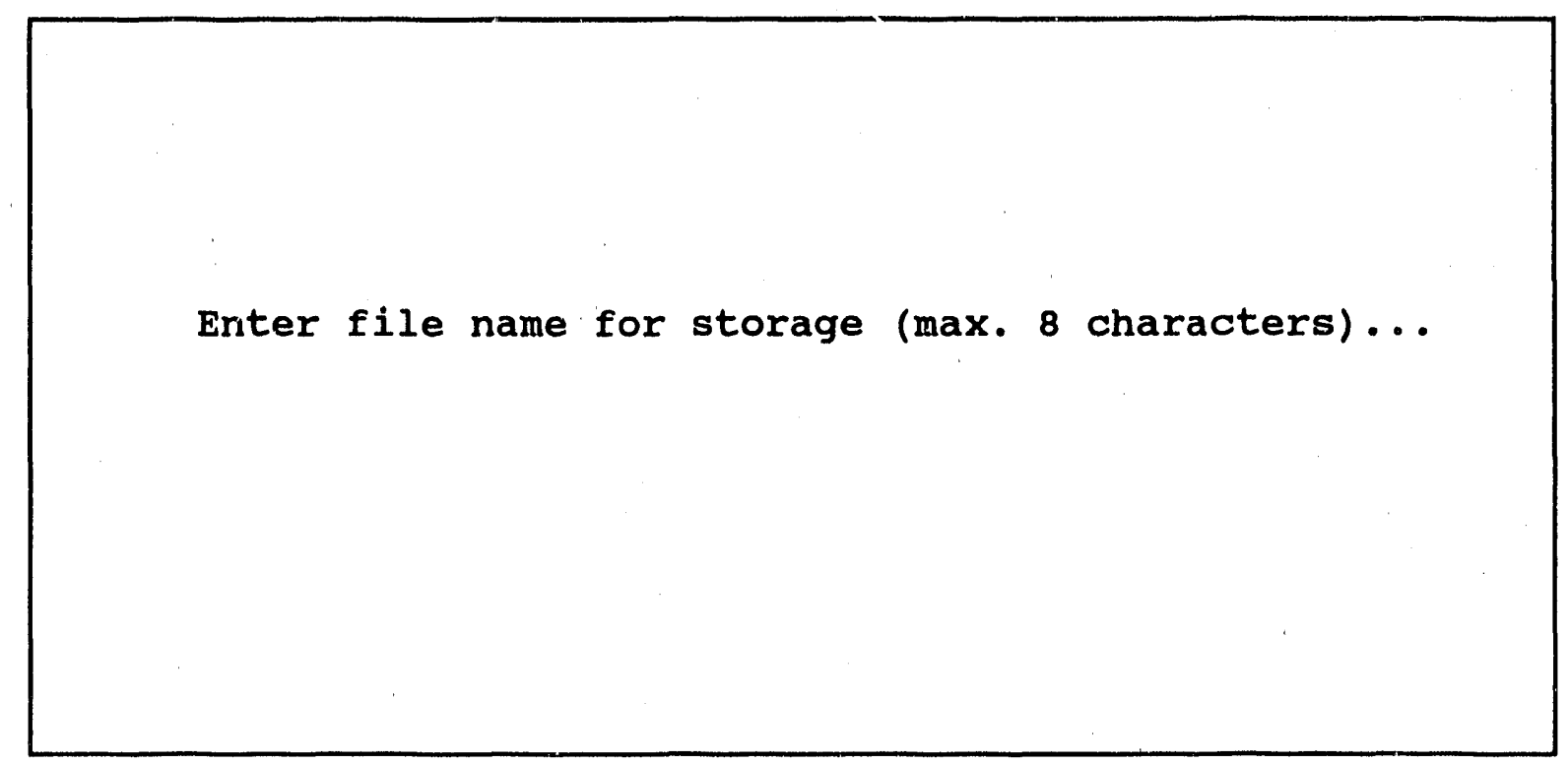

\section{EXPLANATION}

Enter the name of the file to be used in storing the data. It cannot be more than 8 characters long and may consist of numbers and/or letters. When the file is entered and stored, the program returns to the MAIN MENU. 
SECTION F

\section{PRINT A FINAL DATA SET}




\section{SCREEN $1 F$}

Use the cursor keys to highlight your choice, then press enter to choose it.

Enter new (raw) data

Enter data as CFL vs Time

Retrieve a data set

store a data set

print a final data set

Edit data

To add data to a file

Plot data

Plot comparison

Determine De at Temp.

Extend modeled curve

Exit program

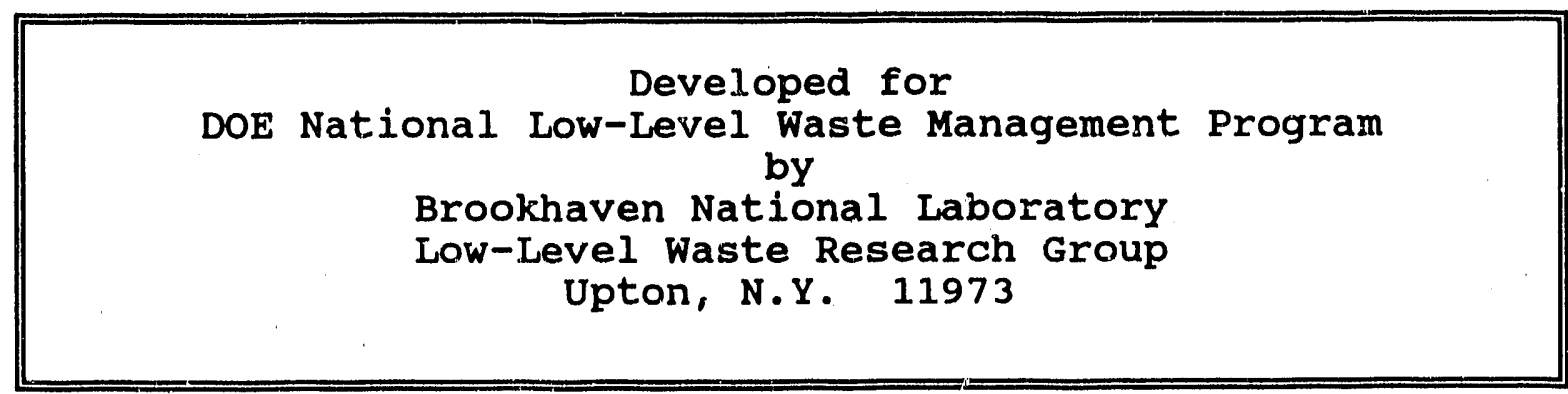

Press F1. for help and definitions

\section{EXPLANATION}

Print a final data set allows the user to either view the file currently in the working memory or to print it out. 


\section{SCREEN 2F}

On screen (S) or printer (P)? [S]/P

\section{EXPLANATION}

Enter "S" or just press ENTER to view the file on the screen. Each individual data set within the file (e.g., each of 3 replicates) will be displayed in sequence. The user can advance to the next data set by pressing any key.

Enter "P" to print out the entire data file.

If no data file is present, this message appears: "There is nothing to print yet.

Press any key to continue." When any key is pressed, the user is returned to the MAIN MENU. 


\section{SCREEN 3F}

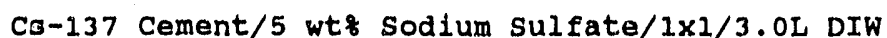
Leaching data for $A$

\begin{tabular}{rccc}
$\#$ & Time(d) & IFL & CFL \\
\cline { 2 - 2 } 1 & 0.08 & & $\frac{7.09 E-02}{7.09 E-02}$ \\
2 & 0.29 & $6.39 \mathrm{E}-02$ & $1.35 \mathrm{E}-01$ \\
3 & 1.00 & $1.30 \mathrm{E}-01$ & $2.65 \mathrm{E}-01$ \\
4 & 2.00 & $1.03 \mathrm{E}-01$ & $3.68 \mathrm{E}-01$ \\
5 & 3.00 & $6.42 \mathrm{E}-02$ & $4.32 \mathrm{E}-01$ \\
6 & 4.00 & $5.40 \mathrm{E}-02$ & $4.86 \mathrm{E}-01$ \\
7 & 5.00 & $3.58 \mathrm{E}-02$ & $5.22 \mathrm{E}-01$ \\
8 & 6.00 & $3.51 \mathrm{E}-02$ & $5.57 \mathrm{E}-01$ \\
9 & 7.00 & $2.89 \mathrm{E}-02$ & $5.86 \mathrm{E}-01$ \\
10 & 8.00 & $2.23 \mathrm{E}-02$ & $6.08 \mathrm{E}-01$ \\
11 & 9.00 & $2.06 \mathrm{E}-02$ & $6.29 \mathrm{E}-01$ \\
12 & 10.00 & $1.43 \mathrm{E}-02$ & $6.43 \mathrm{E}-01$ \\
13 & 11.00 & $1.72 \mathrm{E}-02$ & $6.60 \mathrm{E}-01$
\end{tabular}

Diffusion Model

Diffusion coefficient = 1.33E-07

$Y$-axis intercept $=$

$-0.24 \mathrm{E}-02$

relative error in fit $=$ 0.55 \&

Dissolution Model

standard deviation=

$3.78 \mathrm{E}-02$

average IFI=

4.78E-02

variance $=$

79.05

Partition Model

Diffusion coefficient $=$ 2. 34E-07

Partition Constant $=$

0.82

relative error in fit $=$ $0.16 \%$

\section{EXPLANATION}

This is an example of a data set from a file. Press any key to put the next data set in the file on the screen. When no more sets are available, the user is returned to the MAIN MENU.

The screen displays both data and the parameter values that describe each of the three models. The "relative error in fit" value can be compared to determine which of the models best describes the data. Values of $0.50 \%$ or less are acceptable for the diffusion model and the partition model. A variance of $10 \%$ or less indicates that the dissolution model is acceptable. 


\section{SECTION G}

EDIT DATA 


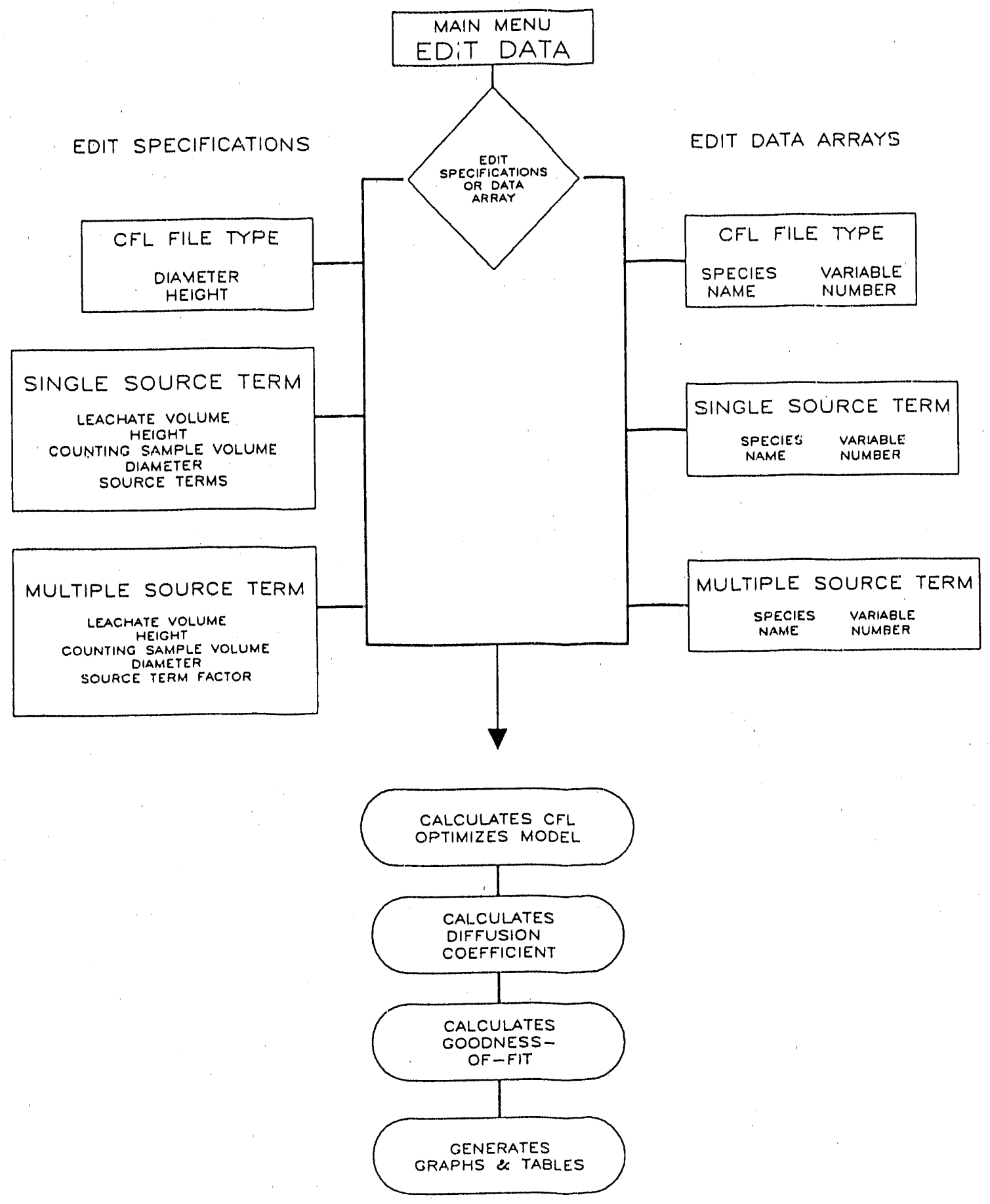




\section{SCREEN 1G}

Use the cursor keys to highlight your choice, then press enter to choose it.

Enter new (raw) data

Enter data as CFL vs Time

Retrieve a data set

Store a data set

Print final data set

Edit data

To add data to a file

Plot data

Plot comparison

Determine De at Temp.

Extend modeled curve

Exit program

Developed for

DOE National Low-Level Waste Management Program by

Brookhaven National Laboratory

Low-Level Waste Research Group

Upton, N.Y. 11973

Press Fl for help and definitions

\section{EXPLANATION}

Edit Lata is used to make corrections in the various inputs given to the program. You can access the edit function through the "Edit Data" selection on the MA: MENU or inrough the data entry functions, just before the optimization sequence begins. 
Edit sample parameters - . . . . . . enter 1

i.e. height, diameter, source terms, etc.

Edit array data . . . . . . . . . enter 2

To exit editing . . . . . . . . . enter 3 Enter your choice...

\section{EXPLANATION}

The data is arranged into two types: SAMPLE PARAMETERS and ARRAY DATA.

Select the type of data to edit at this screen. The parameters, contained in the files vary according to the form that the data was in when it was entered. Therefore, data entered as CFL will have different parameters to edit than the raw data that was entered.

Parameters consist of items, such as diameter, height, source term factor, and counting sample size. Array data consists of the actual leaching data arranged in arrays of species versus intervals. 
The program will automatically display the prompts necessary for the various types of data. For data that was entered as CFL, use SCREENS $3 G$ to $5 G$. For data that was a sinnle source term, see SCREENS $6 \mathrm{G}$ to $8 \mathrm{G}$. For data with multiple source terms see SCREENS $9 \mathrm{G}$ to $12 \mathrm{G}$. 


\section{SCREEN 3G}

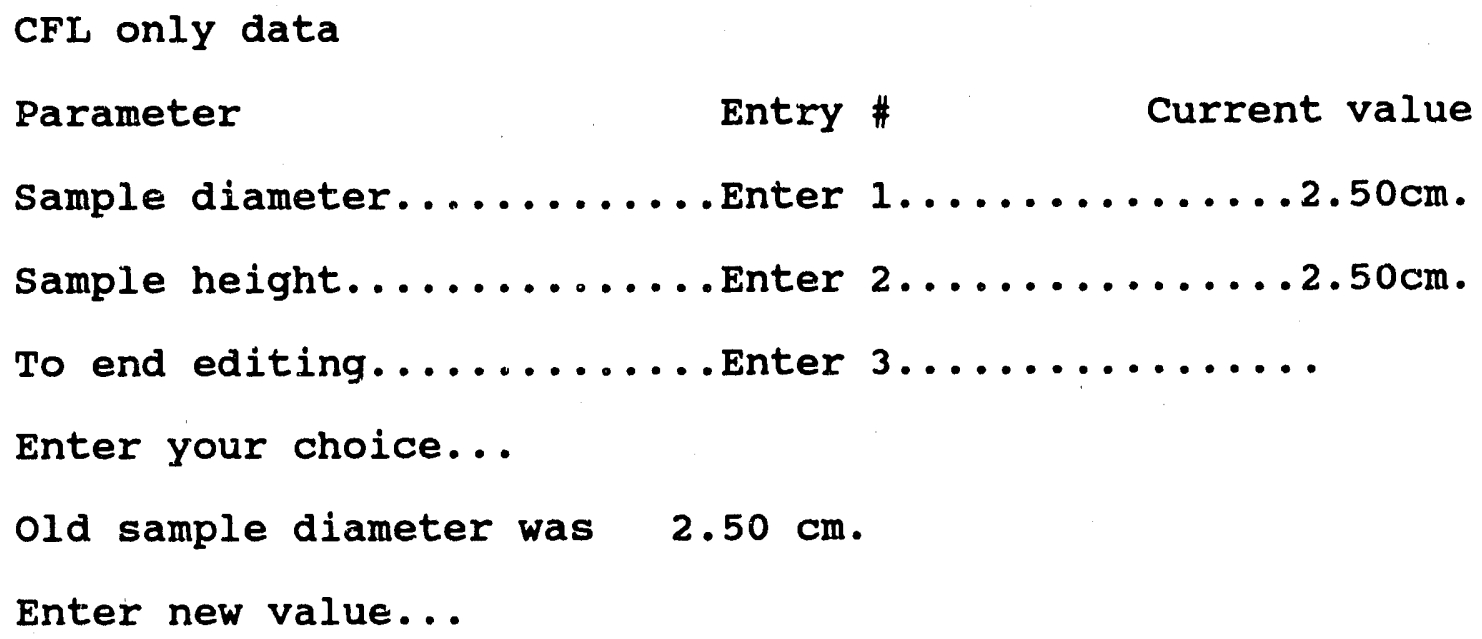

\section{EXPLANATION}

When the data was input as CFL (cumulative fraction leached), the editing function for parameters allows changes only in diameter and height. Other values do not exist in the file. After you provide the answers to the "ENTER YOUR CHOICE" prompt, the program indicates what the old value was and displays the "ENTER NEW VALUE" prompt. After completing any necessary editing, TYPE "3" to end editing the parameters; this returns the user to SCREEN 2G. New values automatically replace old ones in the working file.

The file must be re-optimized (which is done automatically, see SCREEN 13G). If desired, changes can be saved by selecting "Store a data set" in the Main Menu. 


\section{SCREEN 4G}

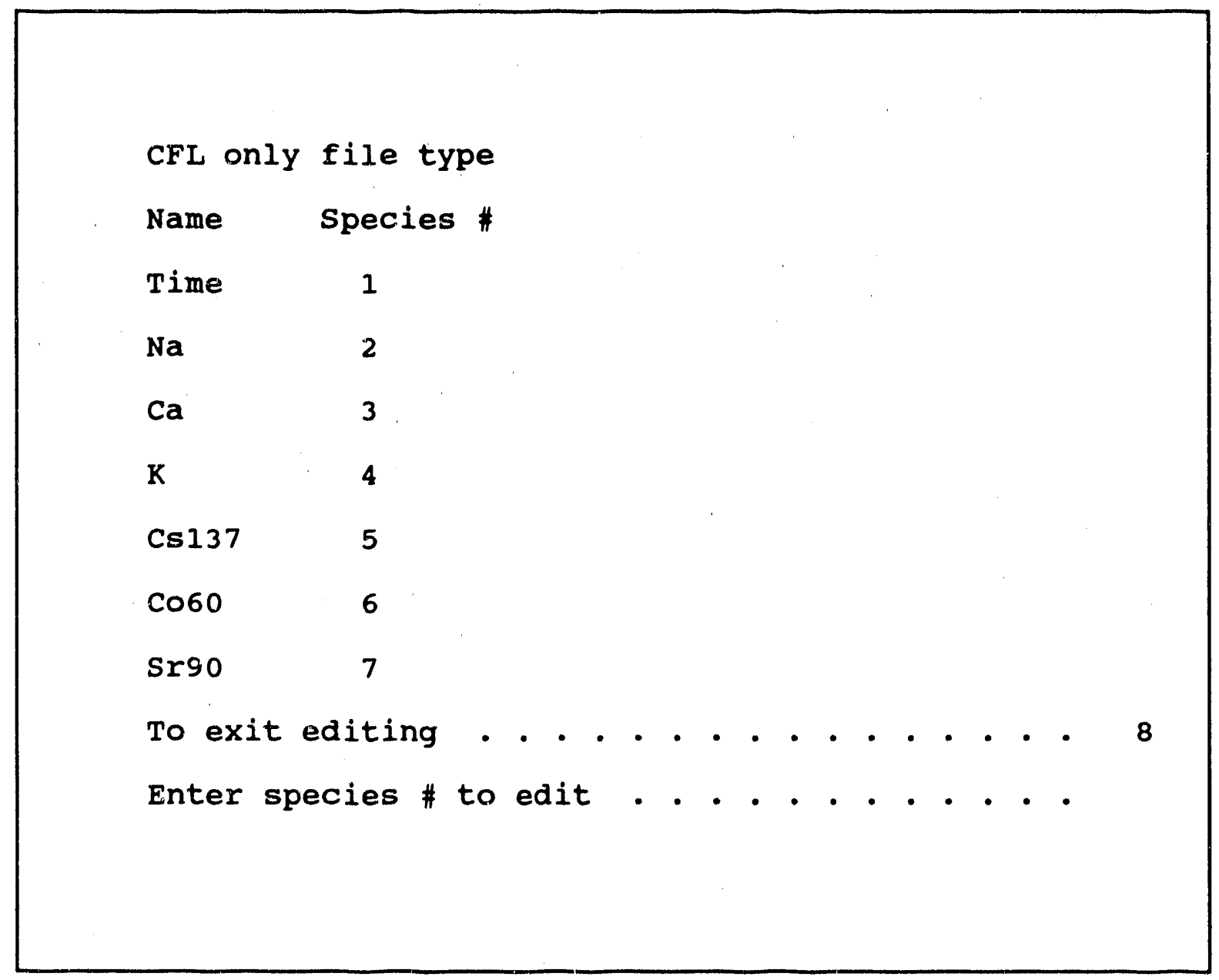

\section{EXPLANATION}

When editing the ARRAY data for data that was input as CFL (cumulative fraction leached), you must define the species that is to be edited. In the example shown above, the species are time and data sets named for various elements and radionuclides. Enter the variable number indicated on the screen to edit that particular species. Type "8" to return to SCREEN 2 G. 


\section{SCREEN 5G}

$\operatorname{Cs} 137$

$\begin{array}{rr}1 & 7.7254 \mathrm{E}-02 \\ 2 & 1.0669 \mathrm{E}-01 \\ 3 & 1.6119 \mathrm{E}-01 \\ 4 & 2.0675 \mathrm{E}-01 \\ 5 & 2.4302 \mathrm{E}-01 \\ 6 & 2.7245 \mathrm{E}-01 \\ 7 & 2.9752 \mathrm{E}-01 \\ 8 & 3.1907 \mathrm{E}-01 \\ 9 & 3.3709 \mathrm{E}-01 \\ 10 & 3.5512 \mathrm{E}-01 \\ 11 & 3.7202 \mathrm{E}-01 \\ 12 & 3.8673 \mathrm{E}-01\end{array}$

Enter 51 to end editing Enter observation \# to change...2

old value was 1..07E-01

Enter new value...

\section{EXPLANATION}

After selecting a species to change in SCREEN $4 G$ (in the case of the example shown above, variable \#5, Cs137, was selected), the appropriate data file is displayed. Enter the observation number (a number from 1 to 13 in this case) to be changed. The program will then display the old value along with a prompt to "ENTER NEW VALUE..." When the "ENTER" key is struck, the new value will replace he old value. Enter "51" to end the array editing function and to return to SCREEN 4G.

The file must be re-optimized (which is done automatically, see SCREEN 13G). If desired, changes can be saved by selecting "Store a data set" in the Main Menu. 


\section{SCREEN 6G}

SINGLE SOURCE TERM DATA

Parameter

Enter \#

Current value

Leachate volume........

Enter $1 \ldots \ldots \ldots .3 .0$

Iiters.

Sample diameter........

Enter $2 \ldots \ldots \ldots \ldots \quad 4.00 \mathrm{~cm}$.

sample height..........

Enter $3 \ldots \ldots \ldots \ldots 7.00 \mathrm{~cm}$.

Source terms......... Enter 4.... (various values)

Counting sample size... Enter 5.... 3.0 millilit.rs.

To end editing....... Enter 6

Enter your choice........2

old sample diameter was $4.00 \mathrm{~cm}$.

Enter new value.........

\section{EXPLANATION}

With data that was entered as New (raw) data, single source term, 5 parameters can be edited, as shown above. Enter the number (Entry \#) of the parameter to be changed. The current values are displayed on the right side of the soreen. For source terms, the current values will be listed when "4" is entered. After the Entry \# of the parameter has been entered, the old value will be displayed on the screen along with the prompt Enter new value...? When the new value has been entered, it will automatically replace the old value in the working file. 
The file must be re-optimized (which is done automatically, see SCREEN 13G). If desired, changes can be saved by selecting "Store a data set" in the Main Menu. 


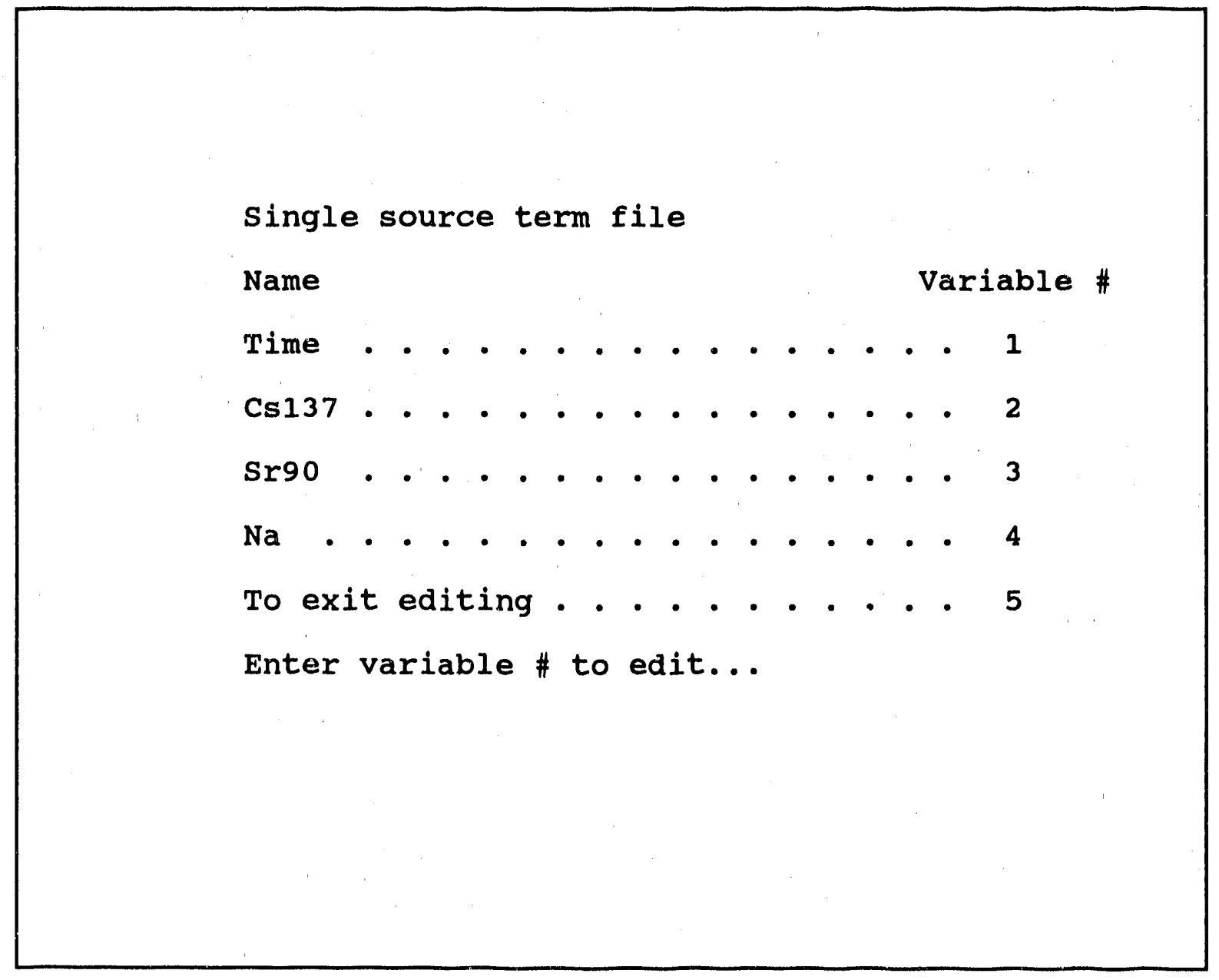

\section{EXPLANATION}

Data stored in an array from single source term files are displayed as shown above. Selecc the name of the species to be edited by entering the appropriate "SPECIES \#." 


\section{SCREEN 8G}

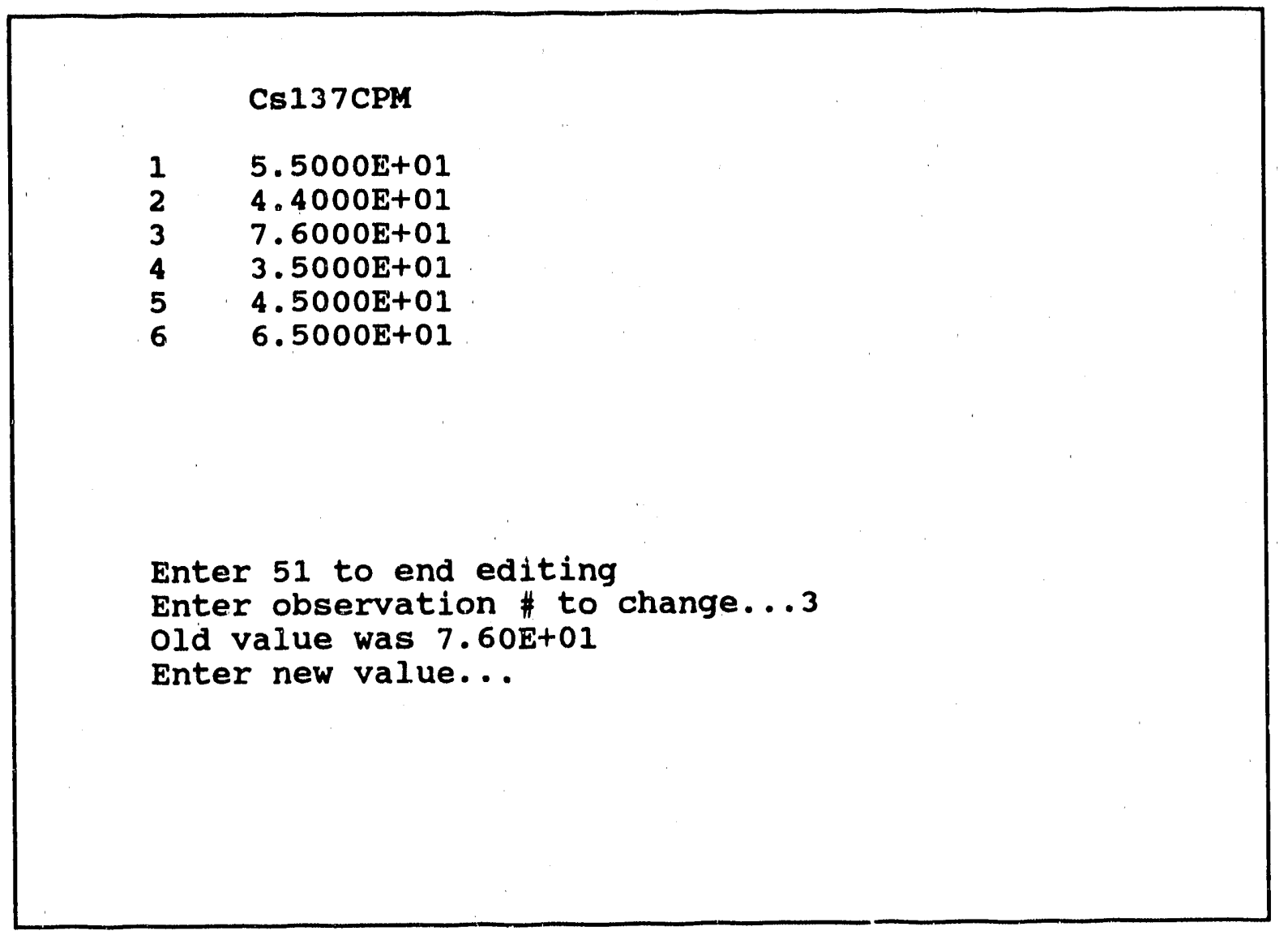

\section{EXPLANATION}

When the species selection is made as shown in SCREEN 7G, the data set for that species is then displayed as shown above.

Enter the observation number (in this case select from 1 to 6 ) to be changed. The old value will be displayed and a prompt to Enter New Value... will appear.

The file must be re-optimized (which is done automatically, seo SCREEN 13G). If desired, changes can be saved by selecting "Store a data set" in the Main Menu. 


\section{SCREEN 9G}

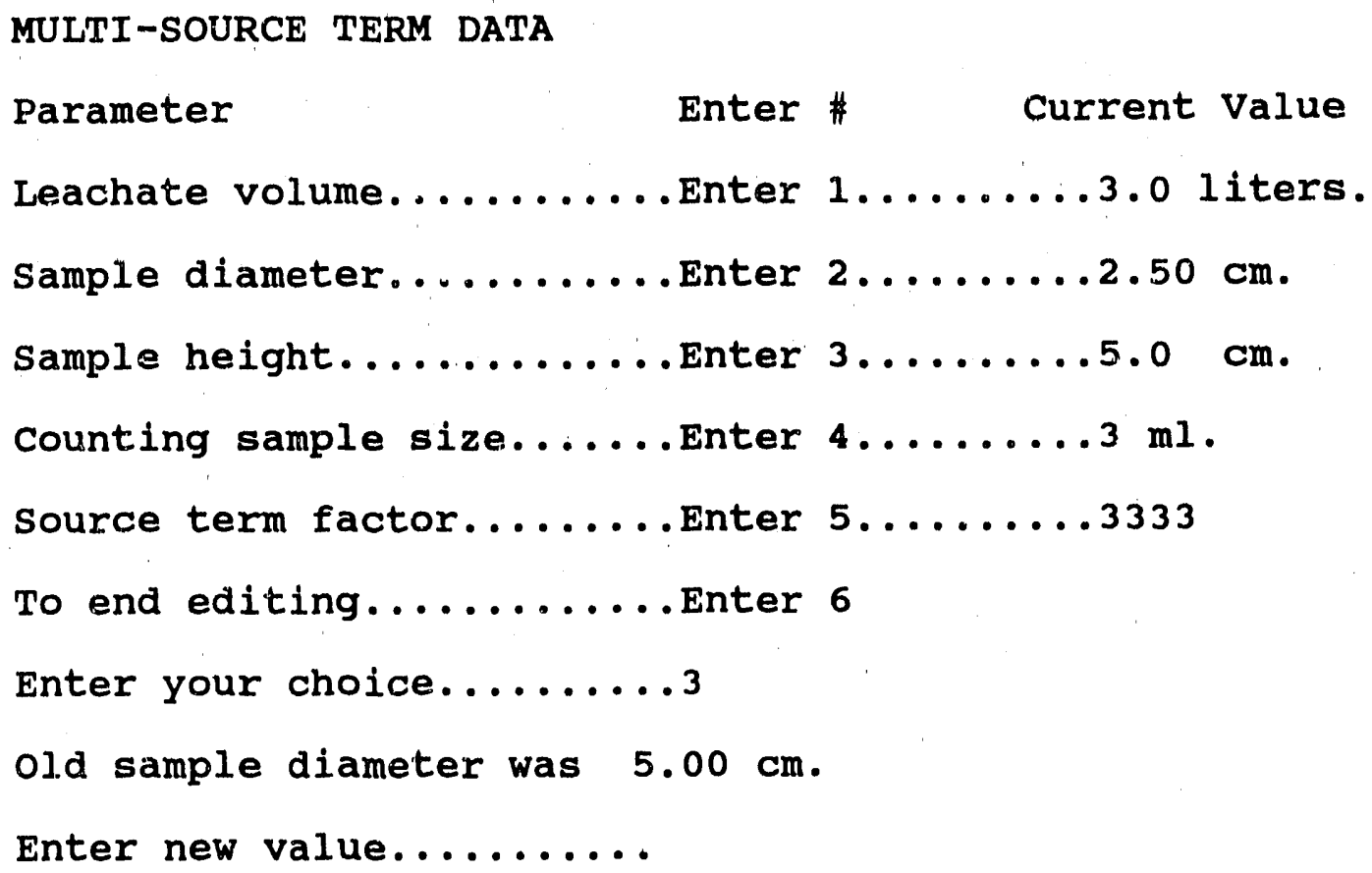

\section{EXPLANATION}

With data that was entered as New (raw) data, multi-source term, 5 parameters can be edited, as shown above. Enter the number (Entry \#) of the parameter to be changed. The current values are displayed on the right side of the screen. After the Entry \# of the parameter has been entered, the old value will be displayed on the screen along with the prompt Enter new value...? When the new value has been entered, it will automatically replace the old value in the working file. The Enter Your 
Choice prompt will remain to allow more editing. Enter "6" to end this editing function.

The file must be re-optimized (which is done automatically, see SCREEN 13G). If desired, changes can be saved by selecting "Store a data set" in the Main Menu. 


\section{SCREEN 10G}

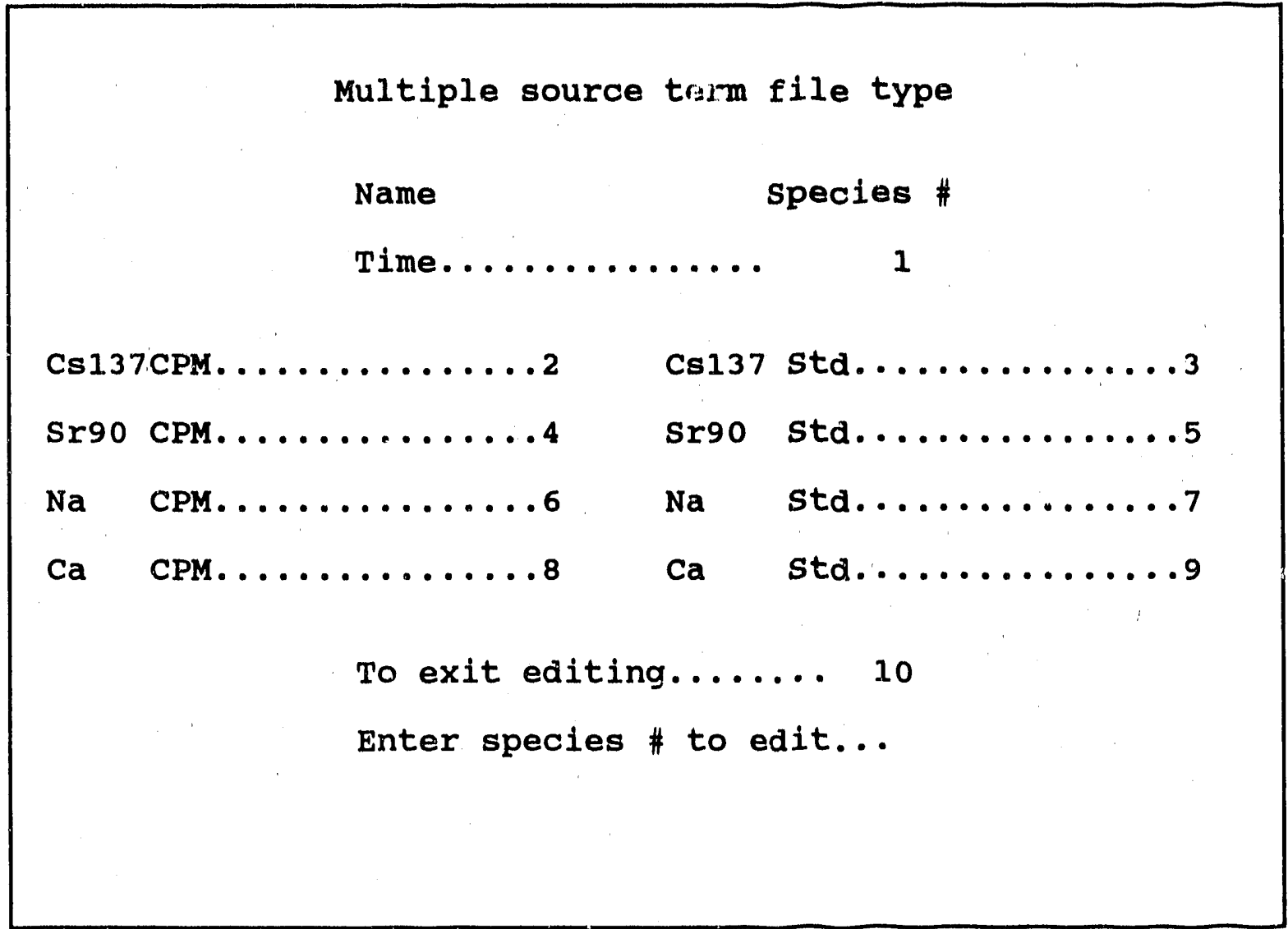

\section{EXPLANATION}

Array data from multiple source term data are displayed as shown in the example above. Select the species to be edited by entering the appropriate "species \#." 


\section{SCREEN 11G}

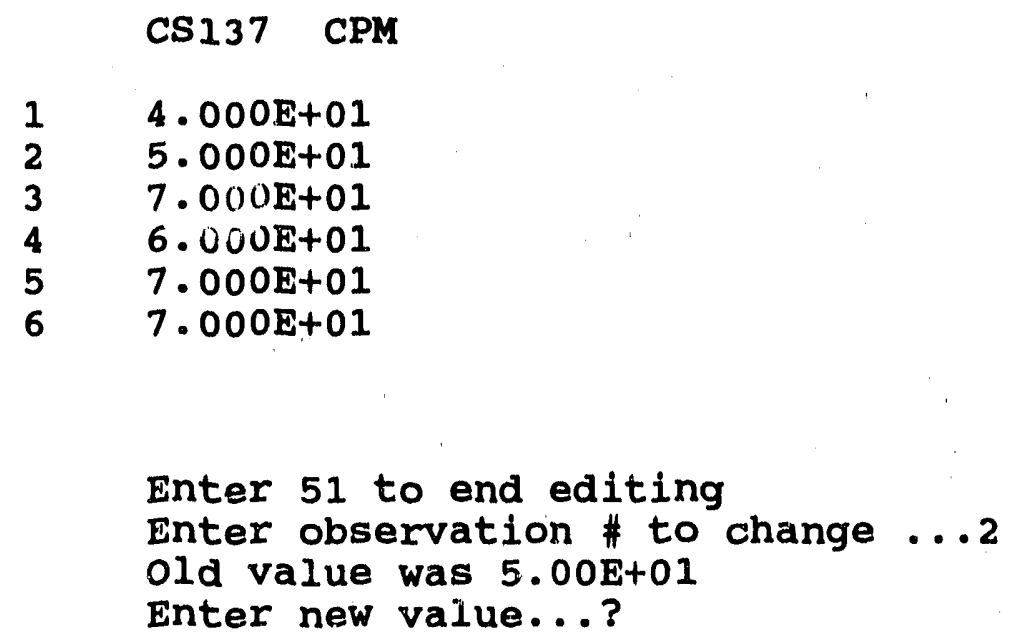

\section{EXPLANATION}

By selecting species \# 2 at SCREEN 10G, the Na data is displayed as shown above. Enter the observation number (in this case a number from 1 to 6) to be changed. The old value will be displayed along with the prompt Enter New Value... When the new value has been entered, it will automatically replace the old value in the working file. Enter observation \# to change...; the prompt will remain to allow more editing until you enter " 51 " to end this editing function.

The file must be re-optimized and saved after changes have been made. 
The modeling function will be performed next. This can be a lengthy process. If any data entry errors were made, you should correct them now to avoid re-running the optimization.

Do you wish to edit the data? $Y[N]$

\section{EXPLANATION}

In all cases, after you finish editing, the program displays the message shown above. This prompt allows you to return to editing if necessary; if editing is not needed, the data set is automatically re-optirnized. 


\section{SCREEN 13G}

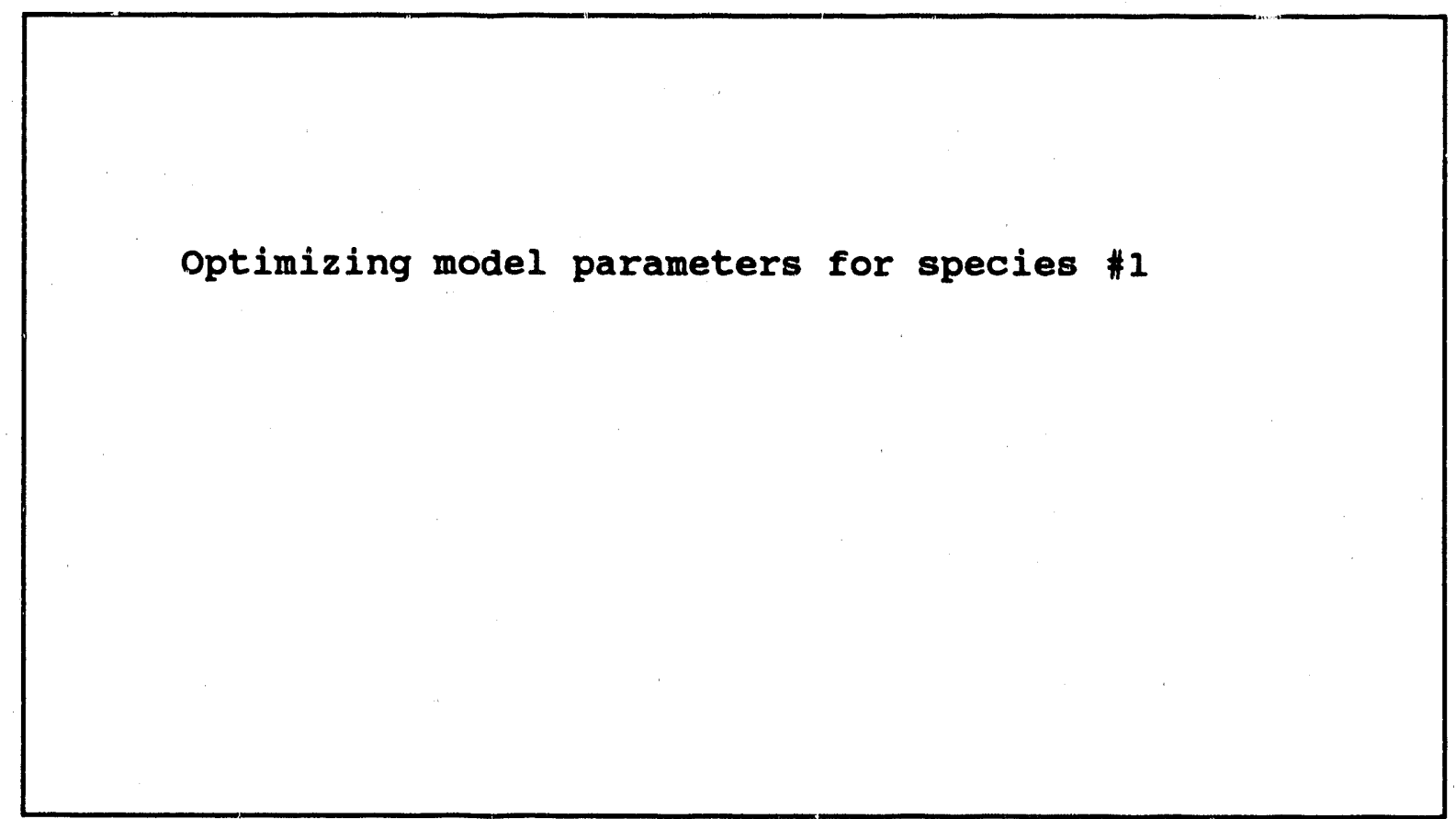

\section{EXPLANATION}

This prompt indicates that calculations are being performed. A rapidly changing number will appear under the line of type as the calculations proceed. This number is increased each time the program completes one set of summations for the model. When the calculations are completed, the program returns to the MAIN MENU. 


\section{SECTION H}

\section{TO ADD DATA TO A FILE}




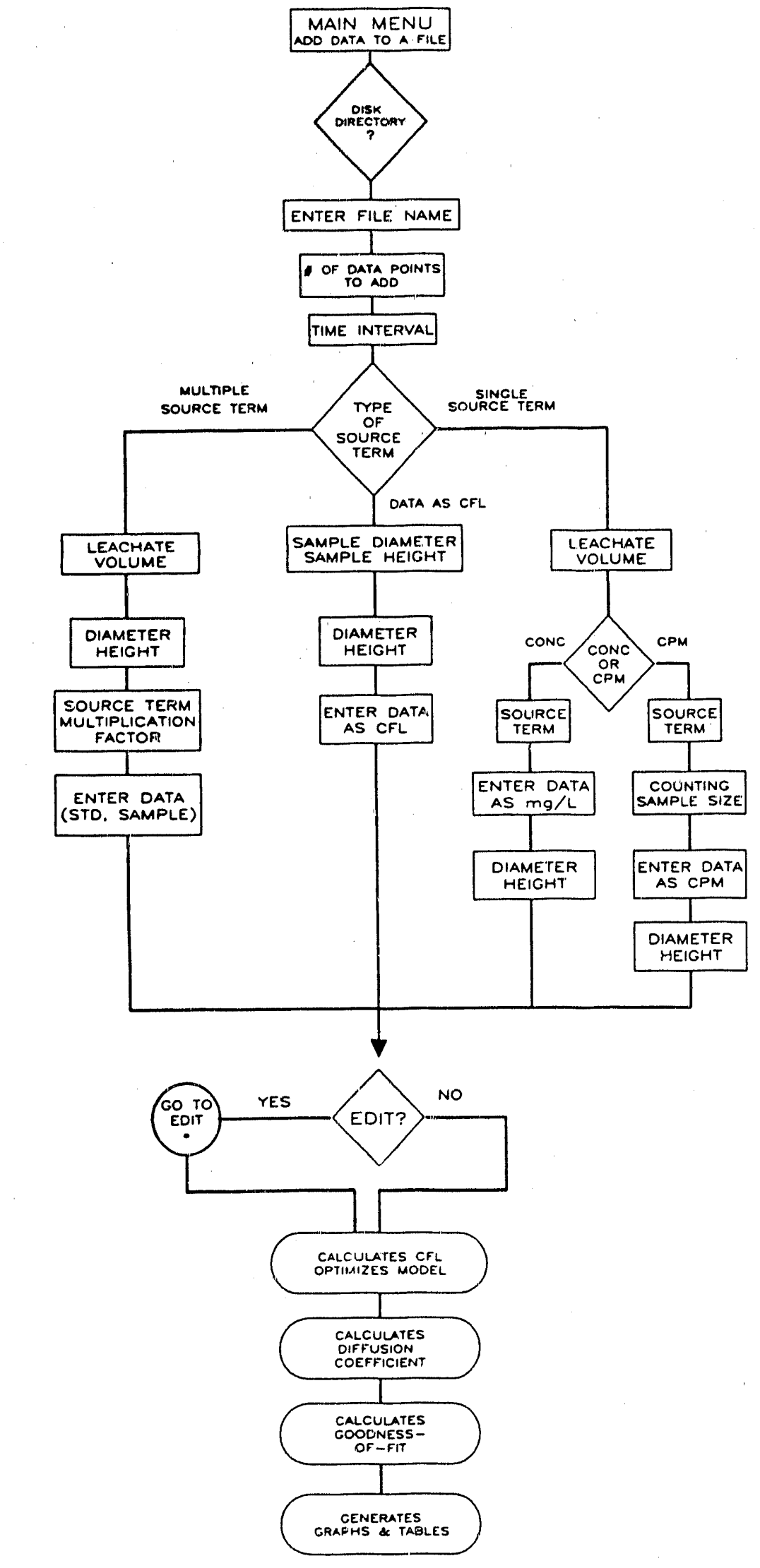

- For Edit function see section G 


\section{SCREEN 1H}

Use the cursor keys to highlight your choice, then press enter to choose it.

Enter new (raw) data

Enter data as CFL vs Time

Retrieve a data set

store a data set

Print a final data set

Edit data

To add data to a file

plot data

Plot comparison

Determine De at Temp.

Extend modeled curve.

Exit program

Developed ror

DOE National Low-Level Waste Management Program by

Brookhaven National Laboratory

Low-Level Waste Research Group

Upton, N.Y. 11973

Press F1 for help and definitions

\section{EXPLANATION}

Use this function to add data to files that previously have been stored with the "ALT" program. The program allows the user to place additional data points after the last one that is already in the file. 


\section{SCREEN $2 H$}

This section is used to add data to a previously stored file

Directory of disk? $Y /[N] /$ Exit/Change (directory)...

\section{EXPLANATION}

Since this function is only used to update data that was previously stored, the file must be taken from either a floppy disk or a hard disk.

In this screen, the program asks if the user needs to view a directory of the disk. If " " is entered, a directory is displayed on the screen. If " $\mathrm{N}$ " is entered, the program proceeds to SCREEN $3 \mathrm{H}$. 


\section{SCREEN 3H}

Enter file name [max. 8 characters.]...

\section{EXPLANATION}

Enter the name of the file to be called up from the disk. A maximum of 8 characters is allowed. 
Enter \# of sampling increments to add...

\section{EXPLANATION}

Enter the number of sampling increments that are to be added to the existing data set. 


\section{SCREEN 5H}

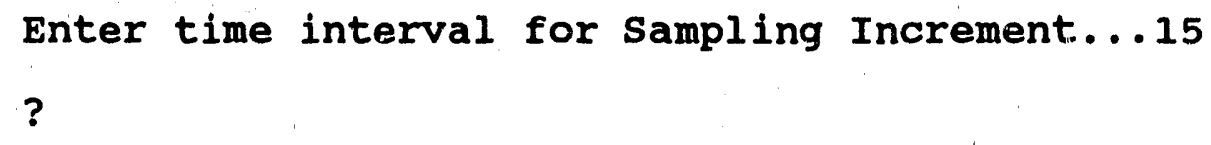

\section{EXPLANATION}

Enter the amount of time (in days) between the last sampling increment and the one being entered. In the example above, the prompt is for sampling increment 15 . This value will be increased automatically based on the last interval contained in the existing file and the number of data points entered at SCREEN $5 \mathrm{H}$. 


\section{SCREEN 6H}

Is new data from $\begin{aligned} & \text { (1) single source term } \\ & \text { (2) Multi-source } \\ & \text { (3) CFL orly }\end{aligned}$
Enter your choice...

\section{EXPLANATION}

Enter the form in which the new data will be input. It does not matter what form the previously stored data is in.

Use (1) "Single source term", when a number of data points for the same species use a precalculated source term than can be entered as a discrete quantity. See SCREENS $7 \mathrm{H}-13 \mathrm{H}$.

Use (2) "Multi-source", when each new data point requires its own accompanying reference value from which the source term is recalculated (this is most often used with short half-life radioisotopes). See SCREENS $14 \mathrm{H}$ to $19 \mathrm{H}$.

Use (3) "CFL only", when the data is to be entered as cumulative fraction leached, and therefore, requires little additional data from which calculations can be performed. See SCREENS $20 \mathrm{H}$ to $23 \mathrm{H}$. 


\section{SCREEN 7H}

Enter Leachate volume (1iters) ...

\section{EXPLANATION}

This screen appears only for single source term data.

Enter the volume of leachate used during the sampling interval. The standard volume for the Accelerated Leach Test is $\mathbf{3 . 0}$ liters. 


\section{SCREEN 8H}

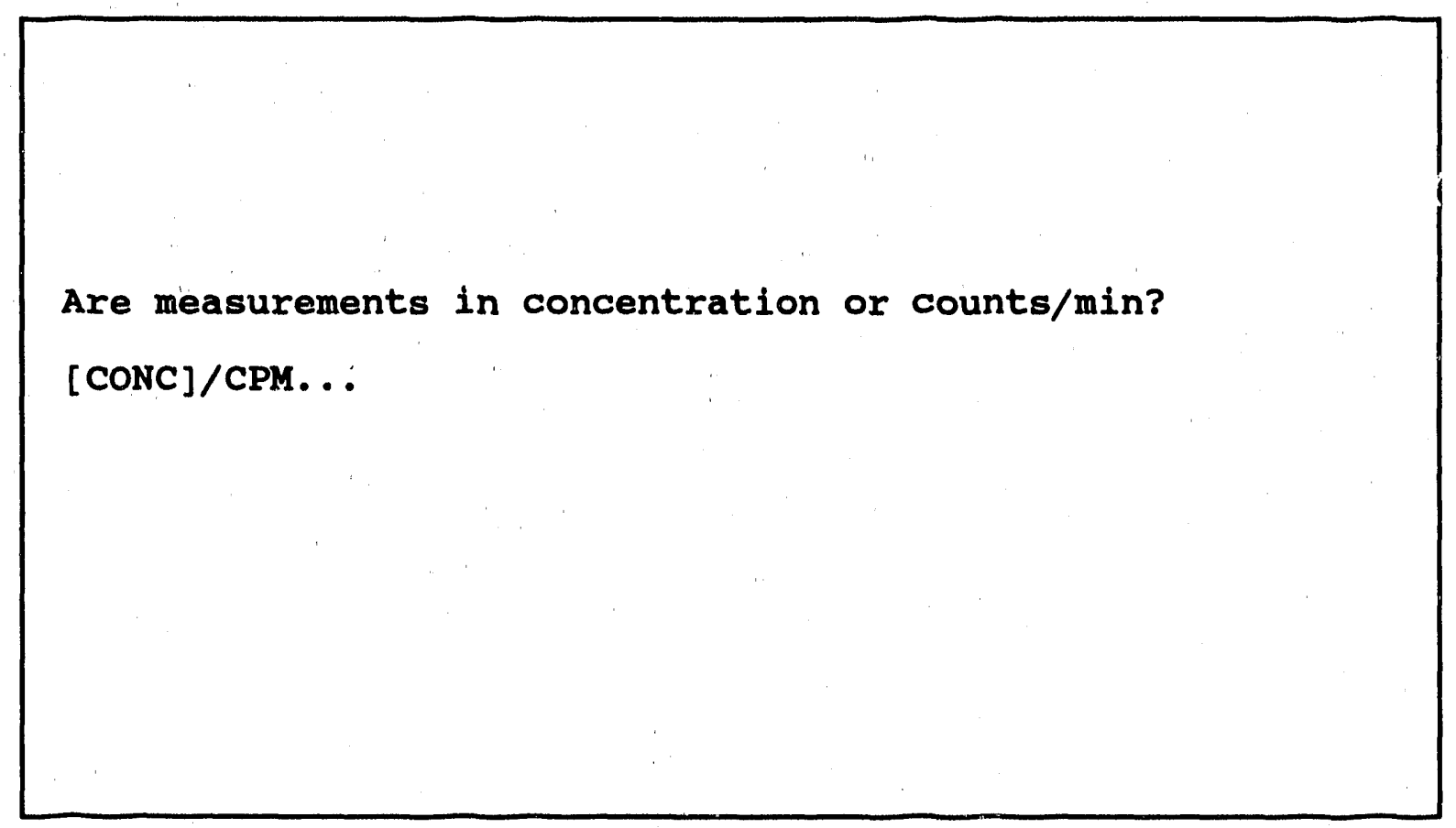

\section{EXPLANATION}

This screen appears only for single source term data.

Calculations are slightly different if the data is in counts-per-minute or in concentration (mg/l). The following screens are for CPM, but "CONC" will also be discussed in the explanations. 


\section{SCREEN 9H}

$$
\text { Enter source Term (CPM) for species... Csi37 }
$$

\section{EXPLANATION}

This screen appears only for single source term data.

Enter the total original amount of activity at the start of leaching for the species indicated in the prompt. For radioactive material, this is entered as counts-per-minute. For data that are entered as concentration, enter the source term as milligrams. 


\section{SCREEN 10H}

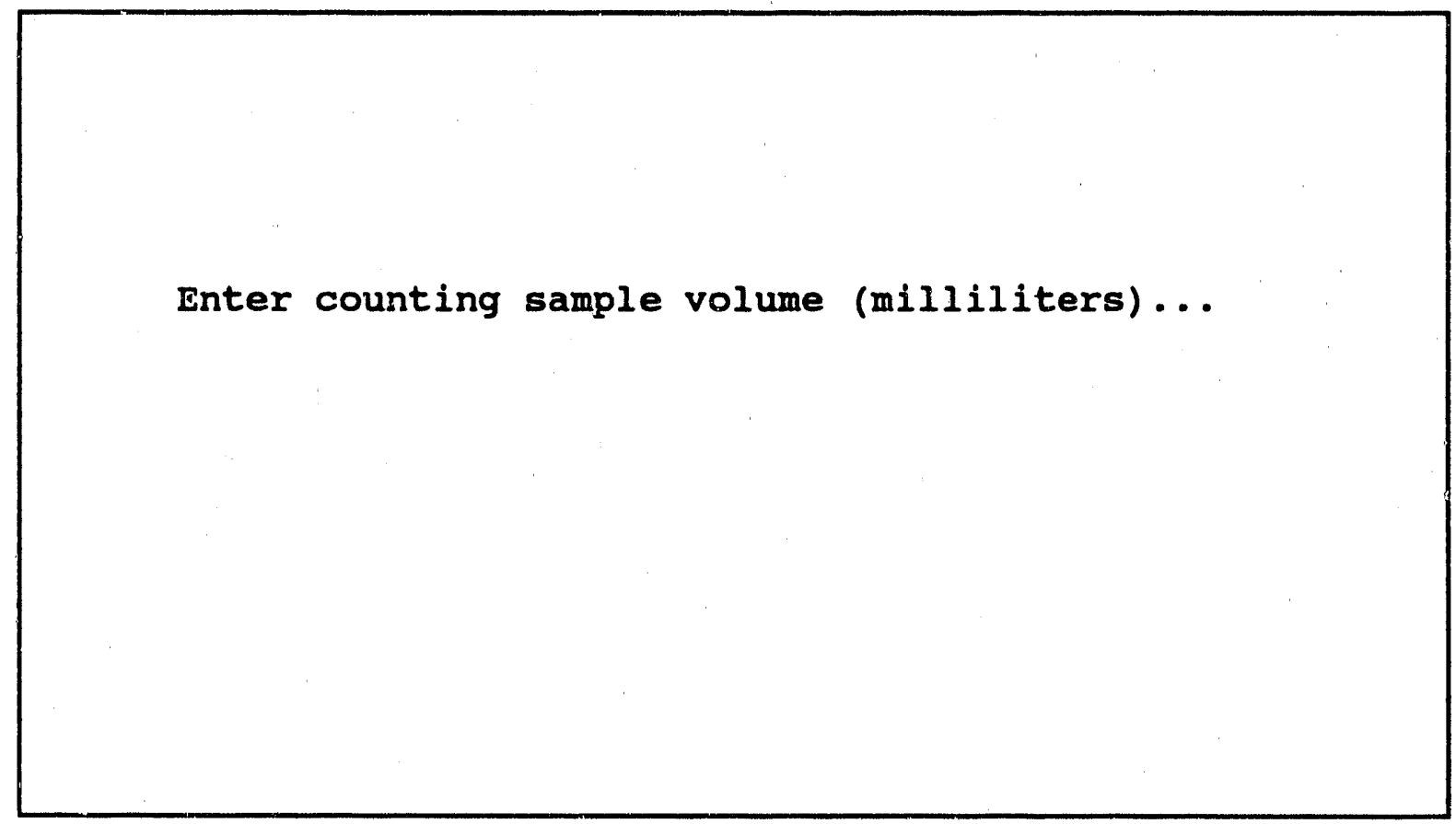

\section{EXPLANATION}

This screen app.jars only for single source term data.

For data that are entered as "CPM" it is necessary to know the volume 0 , aliquot that is analyzed. No equivalent prompt appears for data entered as "CONC." 


\section{SCREEN $11 H$}

Enter data as counts-per-minute for Cs137 at increment. \#14 ?

\section{EXPLANATION}

This screen appears only for single source term data.

For data entered as "CPM," the prompt shown above will appear indicating the species being entered and the increment number. This information on the prompt is updated as data is entered.

For data entered as "CONC," the prompt will be similar but it will ask for concentration $(\mathrm{mg} / \mathrm{l})$. 


\title{
SCREEN 12H
}

\author{
Enter sample diameter (cm)...? \\ Enter sample height (cm) ...?
}

\section{EXPLANATION}

This screen appears only for single source term data.

For data in either form, enter the diameter of the solid sample in centimeters. At the next prompt, enter the height of the solid sample in centimeters. 


\section{SCREEN 13H}

The modeling function will be performed next.

This can be a lengthy process. If any data entry errors were made you should correct them now to avoid re-running the optimization.

Do you wish to edit the data? $Y /[N]$

\section{EXPLANATION}

This screen appears only for single source term data.

At the end of the "to add date to a file" function, the file is re-optimized. The option is given to edit the data. If you select " $N$," the program re-optimizes the model to calculate the best-fit diffusion coefficient.

The new file must be saved manually through the Main Menu. You can save it after the modeling function is finished. 


\section{SCREEN 14H}

$$
\text { Optimizing modeling parameters for species \#1 }
$$

\section{EXPLANATION}

This prompt indicates that calculations are being performed. A rapidly changing number will appear under the line of type as the calculations proceed. This number is increased each time the program completes one set of summations for the model. When the calculations are completed, the program returns to the MAIN MENU. 


\section{SCREEN 15H}

Enter Leachate volume (liters)...

\section{EXPLANATION}

This screen appears only for multiple source term data.

Enter the volume of leachate used during the sampling interval. The standard volume for the Accelerated Leach Test is 3.0 liters. 


\title{
SCREEN 16H
}

\author{
Enter sample diameter (cm)...? \\ Enter sample height $(\mathrm{cm}) \ldots$ ?
}

\section{EXPLANATION}

This screen appears only for multiple source term data.

$\bullet$

Enter the diameter of the solid sample in centimeters. At the next prompt, enter the height of the solid sample in centimeters. 


\section{SCREEN 17H}

Enter source term multiplication factor...?

\section{EXPLANATION}

This screen appears only for multiple source term data.

Enter the source term multiplication factor. This is the factor by which the original source solution was diluted to make the counting standard. For example: $3 \mathrm{ml}$ of tracer were added to a specimen when it was made, and $1 \mathrm{ml}$ of that solution was diluted 1000 -fold to produce the standard that was counted. The multiplication factor would be 3000 . 


\section{SCREEN 18H}

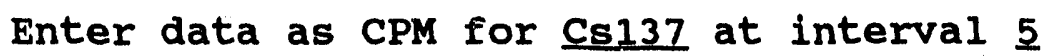

Input standard (CPM), sample (CPM)

\section{EXPLANATION}

This screen appears only for multiple source term data.

The numerical values obtained by radionlivlide analysis are entered as counts per minute (CPM). The first line of the prompt tells what species is being entered and the interval.

Input is in the form of CPM and is entered as the counts for the standard and then the courts for the sample. They are separated by a commr. 


\section{SCREEN 19H}

The modeling function will be performed next.

This can be a lengthy process. If any data entry errors were made you should correct them now to avoid re-running the optimization.

Do you wish to edit the data? $Y /[N]$

\section{EXPLANATION}

At the end of the "to add data to a file" function, the file is re-optimized. The option is given to edit the data. If you select " $\mathrm{N}$, " the program re-optimizes the model to calculate the best-fit diffusion coefficient.

The new file must be saved manually. You can save it through the Main Menu after the modeling function is completed. 


\section{SCREEN 20H}

optinizing model parameters for species \#1

\section{EXPLANATION}

This prompt indicates that calculations are being performed. A rapidly changing number will appear under the line of type as the calculations proceed. This number is increased each time the program completes one set of summations for the model. When the calculations are completed, the program returns to the MAIN MENU. 


\section{SCREEN 21H}

Enter sample diameter (cm) ...?

Enter sample height (cm) ...?

\section{EXPLANATION}

This screen appears for data entered as CFL.

Enter the diameter of the solid sample that was leached in centimeters. At the next prompt, enter the height of the solid sample in centimeters. 


\section{SCREEN 22H}

Enter data for ..... at sampling increment .... Input CFI...

\section{EXPLANATION}

This screen appears only for data entered as CFL.

Enter the data, in the form of cumulative fraction leached (CFL), for each species that is indicated in the prompt for the interval that is indicated. Data must be input as a decimal fraction. 


\section{SCREEN 23H}

The modeling function will be performed next.

This can be a lengthy process. If any data entry errors were made you should correct them now to avoid re-running the optimization.

Do you wish to edit the data? $\mathrm{Y} / \mathrm{N}]$

\section{EXPLANATION}

This screen appears only for data entered at CFL.

At the end of the "add to data file" function, the file is re-optimized. The option is given to edit the data. If you select " $N$," the program re-optimizes the model to calculate the best-fit diffusion coefficient.

The new file must be saved manually; you can save it through thə Main Menu after the modeling function is completed. 


\section{SCREEN 24H}

Optimizing model parameters for species \#1

\section{EXPLANATION}

This prompt indicates that calculations are being performed. A rapidly changing number will appear under the line of type as the calculations proceed. This number is increased each time the program completes one set of summations for the model. When tile calculations are completed, the program returns to the MAIN MENU. 


\section{SECTION I}

PLOT DATA 


\section{SCREEN 11}

Use the cursor keys to highlight your choice, then press enter to choose it.

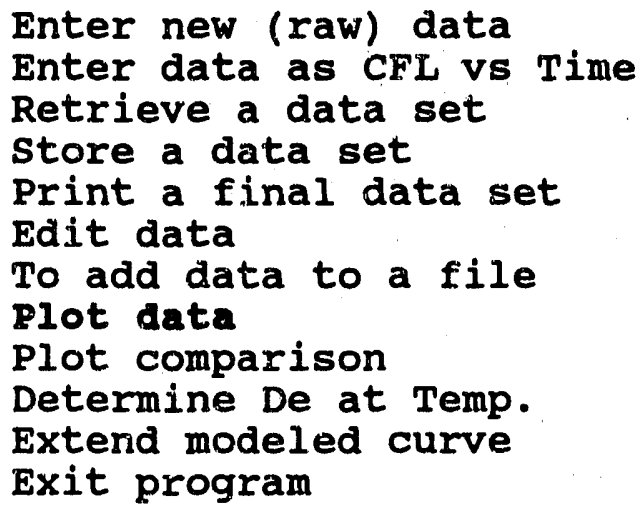

Developed for

DOE National Low-Level Waste Management Program by

Brookhaven National Laboratory

Low-Level Waste Research Group

Upton, N.Y. 11973

Press F1 for help and definitions

\section{EXPLANATION}

This function is used to produce on-screen plots of the data in the form of cumulative fraction leached. Before you select the plotting function, data must be present in the working memory of the program. If no data is present, a warning message will be given and the user will be returned to the MAIN MENU. 


\section{SCREEN 2I}

To plot data using the DIFFUSION MODEL.......enter 1

To plot data using the DISSOLUTION MODEL....enter 2

To plot data using the PARTITION MODEL......enter 3

\section{EXPLANATION}

The user has a choice of three mathematical models, the Diffusion Model, the Dissolution Model, and the Partition Model, which may be used to describe the data. On-screen plots that show the data and the results of the optimized model are generated in this section. To use this function, a data set must already be in the active memory (either by entering the data manually or by retrieving a data set). The user selects the model to be plotted. After the first data set is plotted, other sets on the same file are also plotted by pressing any key. When all data sets have been displayed as plots, the program returns to the MAIN MENU. To plot the same data using one of the other models, select plot data and then choose the desired model. 


\section{SCREEN 31}

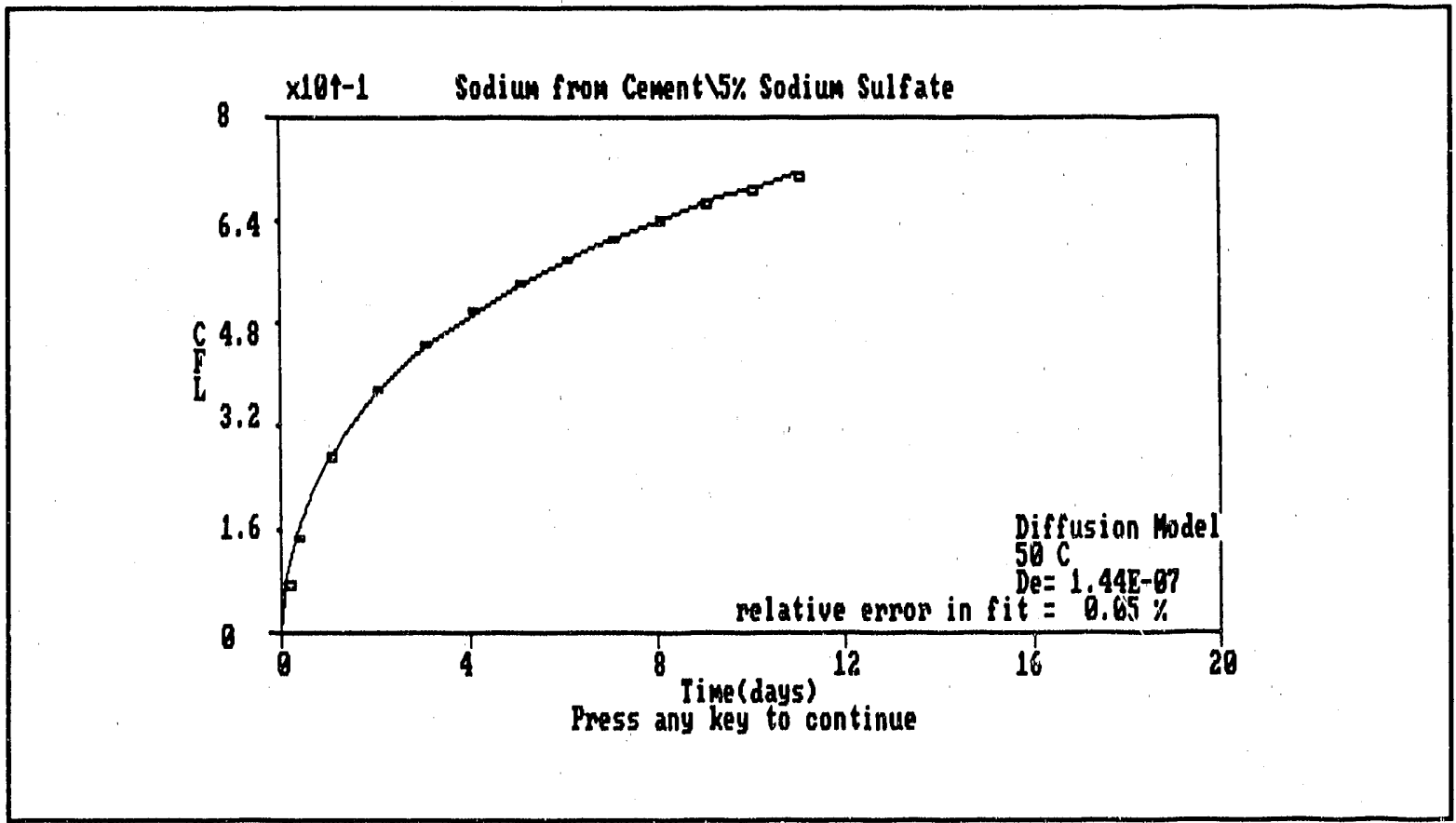

\section{EXPLANATION}

This is an example of a set of data plotted by ALT using the Diffusion Model. It is in the form of cumulative fraction leached plotted against time. The $\mathrm{Y}$-axis values are plotted as multiplied by $\left(10^{-1}\right)$, which is shown at the upper left side of the plot. The program automatically selects ranges of the data to plot. The title (as entered in SCREEN 3A, for example) is printed at the top of the graph. The species of the data (as entered in SCREEN 6A) is printed at the lower right, along with the name of the model, the optimized diffusion coefficient, and the relative error in fit $\left(E_{R}\right)$. The data are plotted as individual points, and the best fit model curve is plotted as a line through the points. Pressing any key will advance the program to the next species that is to be plotted. When plots of all species from the data file have been displayed, the program returns to the MAIN MENU. 


\section{SCREEN 41}

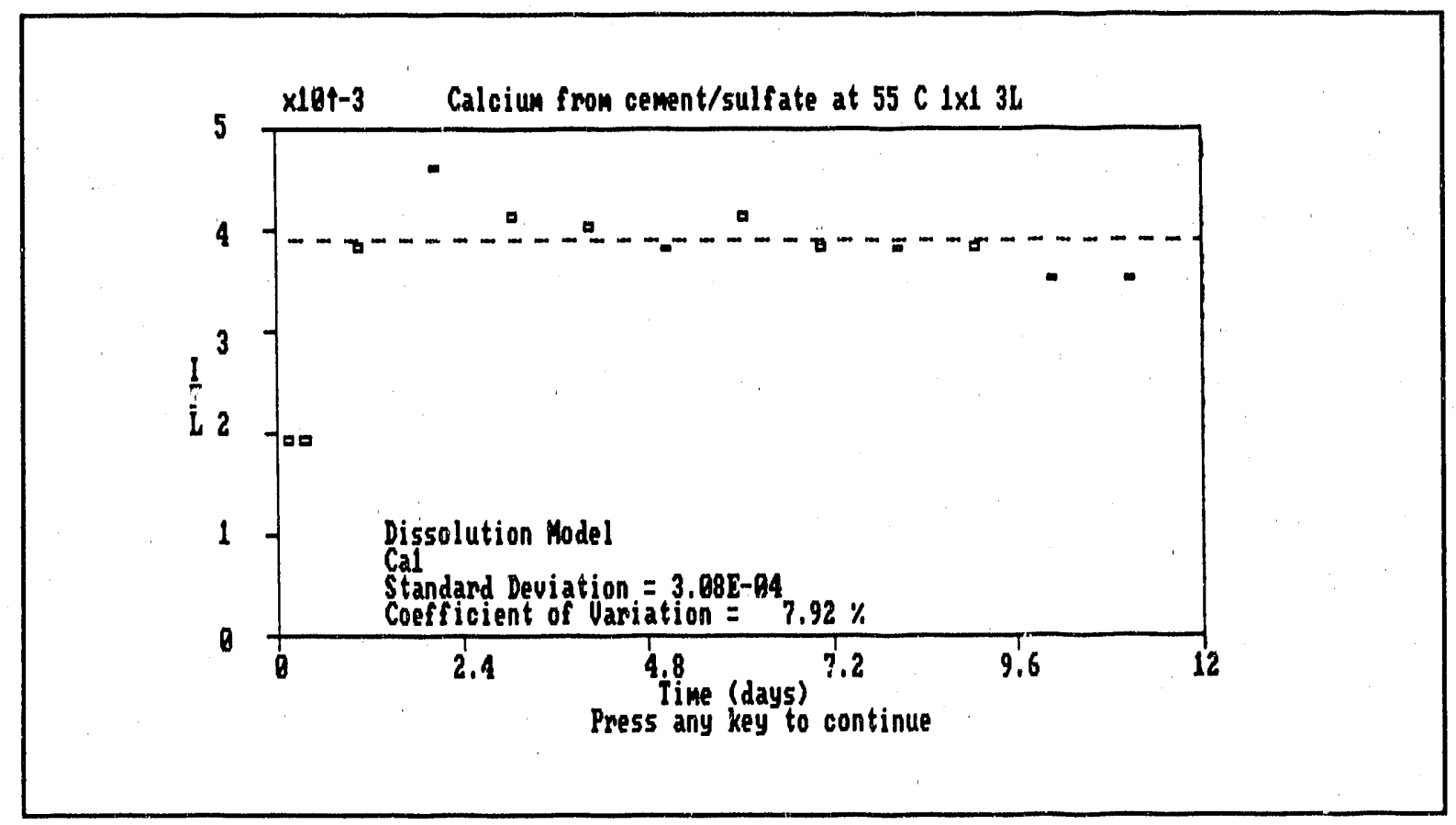

\section{EXPLANATION}

This is an example of a set of data plotted by ALT using the Dissolstion Model. It is in the form of incremental fraction leached plotted against time. The program automatically selects ranges of the data to plot. The title (as entered in SCREEN 3A, for example) is printed at the top of the graph. The species of the data (as entered in SCREEN 6A) is printed at the lower left side as is the mean, the standard deviation, and coefficient of variation of the distribution about the mean. The data are plotted as individual points, and the mean (excluding the first two data points) is shown as a horizontal line. Pressing any key will advance the program to the next species that is to be plotted. When plots of all species from the data file have been displayed, the progry returns to the MAIN MENU. 


\section{SCREEN 5I}

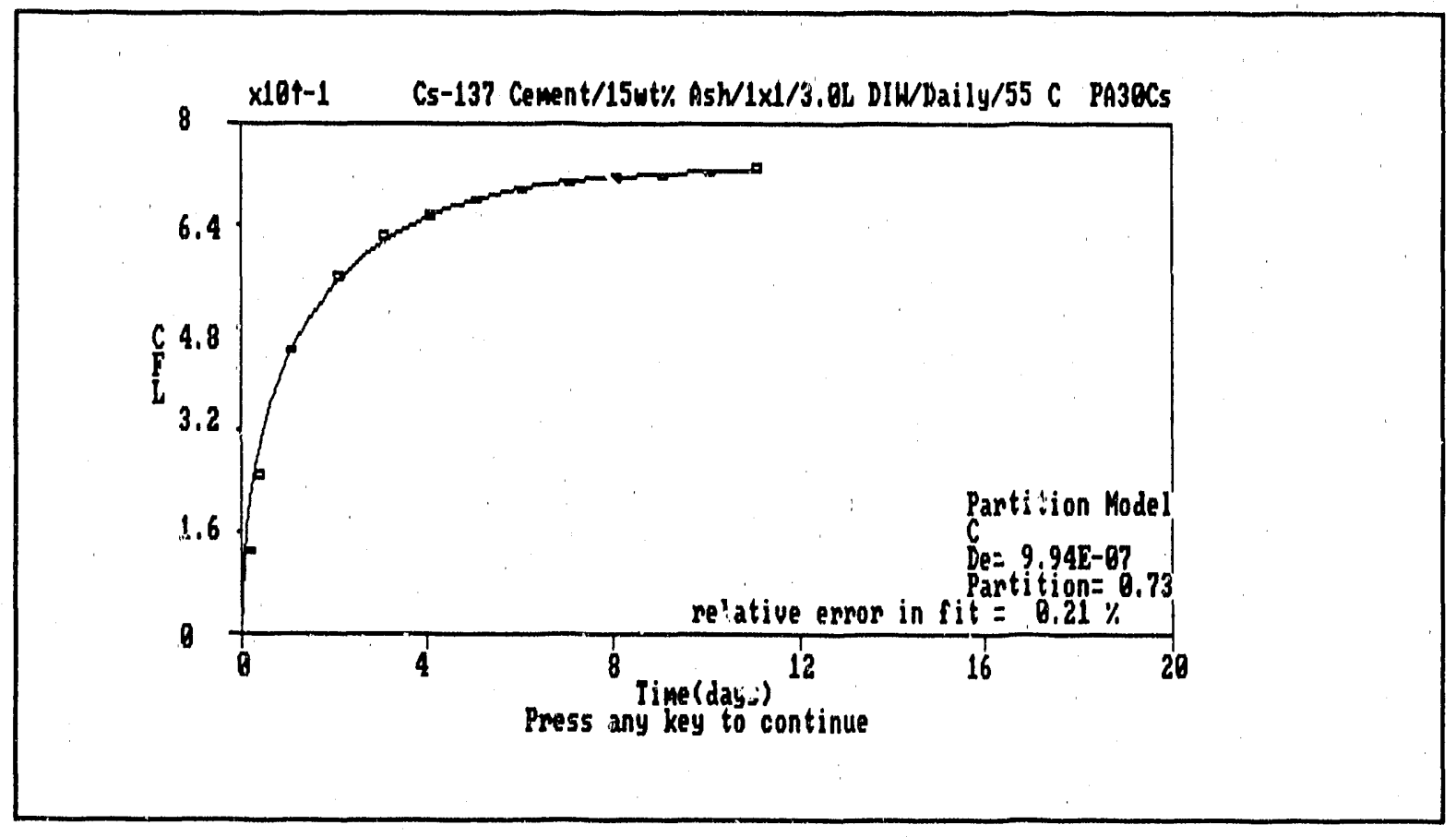

\section{EXPLANATION}

This is an example of a set of data plotted by AL.T using the Partition Model. This model combines partitioning of the source term with the diffusion model. It is in the form of cumulative fraction leached plotted against time. The program automatically selects ranges of the data to plot. The title (as entered in SCREEN 3A, for example) is printed at the top of the graph. The species of the data (as entered in SCREEN 6A) is printed at the lower right, as is the name of the model, the optimized diffusion coefficient, the partition factor and the relative error in fit $\left(E_{R}\right)$. The data are plotted as individual points and a best fit model curve is plotted as a line through the points. Pressing any key will advance the program to the next species that is to be plotted. When plots of all species from the data file have been displayed, the program returns to the MAIN MENU. 


\section{SECTION J}

\section{PLOT COMPARISON}




\section{SCREEN 2J}

To plot Experimental CFL

vs Modeled CFL (Diffusion)......Enter 1

To plot Adjusted CFL

vs Modeled CFL (Partition)......Enter 2

?

\section{EXPLANATION}

This screen allows the user to select between the diffusion model and the partition model to generate plots comparing the model results ( $Y$-Axis) and the experimental data (X-Axis). To use this function a modeled data set must already be in the program's active memory. 


\section{SCREEN 3J}

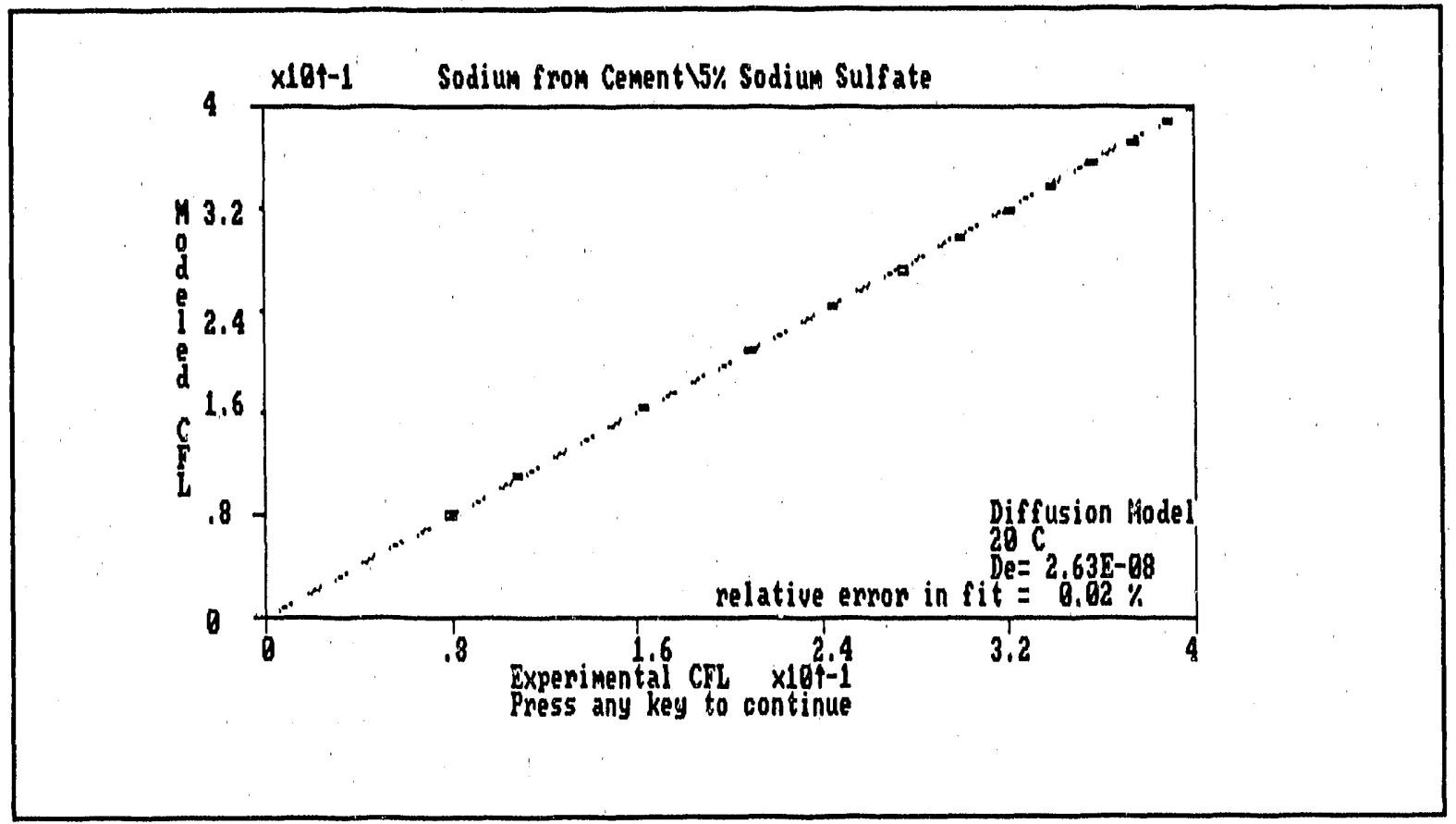

\section{EXPLANATION}

This is an example of a graph generated by the plot comparison function. The cumulative fraction leached (CFL) generated from experimental data is on the $X$-axis while the CFL generated by the model is on the Y-axis. If the fit between the two were perfect, the points would fall exactly on the dotted line. Deviations of the fit can be observed on this plot, showing where the model and experimental data do not correlate very well. The title of the data set is printed at the top of the graph. The species, the best fit diffusion coefficient, and the relative error of the fit between the model and the data are displayed at the lower right of the plot.

The dotted line, indicating a perfect fit, does not necessarily pass through the origin. It is adjusted with a Y-intercept valuэ. 


\section{SECTION K}

\section{DETERMINE $D_{e}$ AT TEMP.}




\section{SCREEN 1K}

Use the cursor keys to highlight your choice, then press enter to choose it,

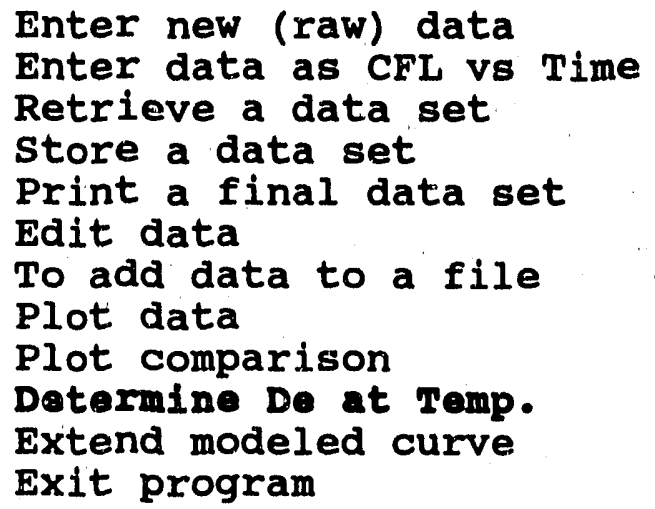

Developed for

DOE National Low-Ievel Waste Management Program by

Brookhaven National Laboratory

Low-Level Waste Research Group

Upton, N.Y. 11973

Press F1 for help and definitions

\section{EXPLANATION}

This function is used to calculate the equation ( $f$ the line and the correlation coefficient for the relationship of log diffusion coefficient $\left(D_{0}\right)$ to reciprocal temperature $(1 / T(K))$. This is the Arrhenius function described by the equation:

$$
D_{0}-A \exp \left(\frac{-E a}{R T}\right)
$$


where $A$ is a pre-exponential factor, $E_{a}$ is the activation energy, $R$ is the gas constant and $T$ is temperature in degrees kelvin. 


\section{SCREEN 2K}

This section is used to calculate an equation of the form

$Y=m X+b$ for Arrhrenius plots of natural $\log (1 n)$ De versus $1 / \mathrm{T}$.

There must be a minimum of three different temperatures.

Enter Temperature (C), Diffusion coefficient (cm/sec...)

Hit return (zero for De) to end data entry.

Input data pair \# $1 .$.

\section{EXPLANATION}

To perform the calculation, the user must enter experimental temperature (in degrees centigrade) and $D_{0}\left(\mathrm{~cm}^{2} / \mathrm{sec}\right)$. A minimum of three data sets, entered as temperature and then $D_{0}$, separated by a comma are required. When all data are entered, press "ENTER" to start the calculations. Entering a value of zero for $D_{0}$ will also end data entry and start the calculations. 


\section{SCREEN 3K}

Enter Temperature $\left({ }^{\circ} \mathrm{C}\right)$, Diffusion coefficient $(\mathrm{cm}) / \mathrm{sec} .$. Hit return (zero for $\mathrm{De}$ ) to end data entry.

For $X=1 / T$ and $Y=\ln (D e)$

$\mathrm{m}=-0.12 \mathrm{E}+05$

$\mathrm{b}=3.20 \mathrm{E}+01$

Correlation coefficient $r=0.970$

Activation Energy, $\mathrm{Ea}=24.3 \mathrm{kcal} / \mathrm{mol}$

Do you wish to determine a De at a different temperature $[\mathrm{Y}] / \mathrm{N} \ldots$

\section{EXPLANATION}

Results are displayed on the screen as numerical values for the components of the equation of the line that best describes the data. The linear correlation coefficient $r$ is given and the activation energy, $\mathrm{E}_{\mathrm{a}}$ (in $\mathrm{kcal} / \mathrm{mole}$ ) is calculated from the slope of the line. If it is desired, enter " $\gamma$ " to calculate $a D_{\theta}$ value for another temperature based on this data. 


\section{SCREEN 4K}

Do you wish to determine a De at a different temperature $[\mathbf{Y}] / \mathbf{N} \ldots$

Enter the temperature $\left({ }^{\circ} \mathrm{C}\right)$ that you want a De for ... 78

$$
\begin{aligned}
& \mathrm{De}=5.76 \mathrm{E}-02 \\
& \text { Another De? } \mathrm{Y}[\mathrm{N}]
\end{aligned}
$$

\section{EXPLANATION}

If you enter "Y," the program will use the calculated best fit line to estimate a new $D_{\theta}$ value for the temperature entered. This calculation is extrapolated from the linear equation used to describe the Arrhenius function. If you enter " $N$," the program returns to the MAIN MENU. 


\section{SECTION L}

EXTEND MODELED CURVE 


\section{SCREEN 2L}

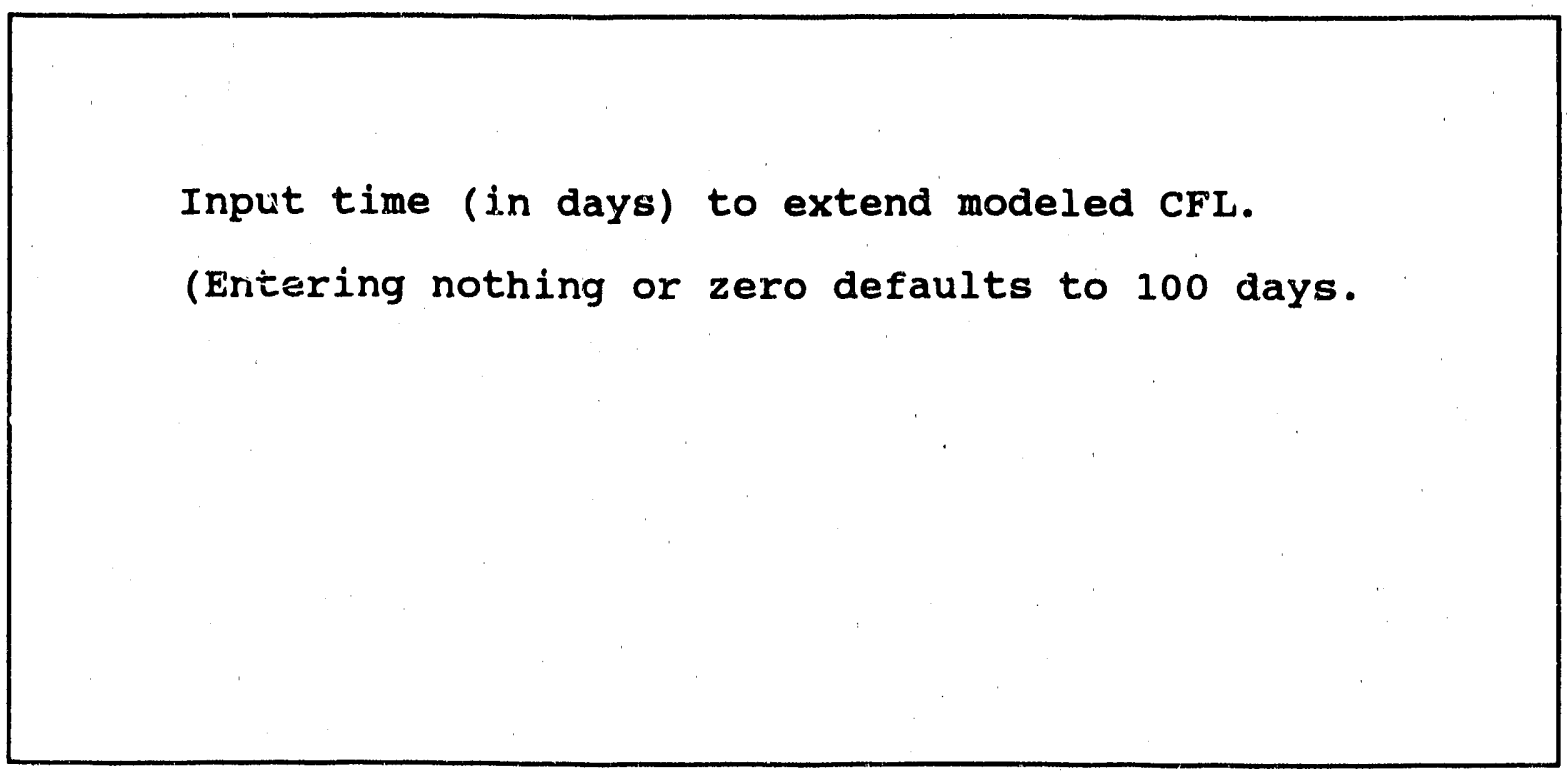

\section{EXPLANATION}

Enter the number of days to which the model is to calculate the diffusion curve. The program automatically defaults to 100 days, and it will use the diffusion coefficient of the data in the memory. 


\section{SCREEN 3L:}

Input time (in days) to extend modeled CFL. (Ent.ering nothing or zero defaults to 100 days.) ... 500

Input diameter, height (in $\mathrm{cm}$ ) to use in modeling (Entering nothing or zero defaults to experimental size)...

\section{EXPLANATION}

After answering the prompt on the previous screen, enter the diameter and height, in centimeters, of the solid sample. The program will default to the size of the sample used in the experimental data held in the working memory. 


\section{SCREEN 4L}

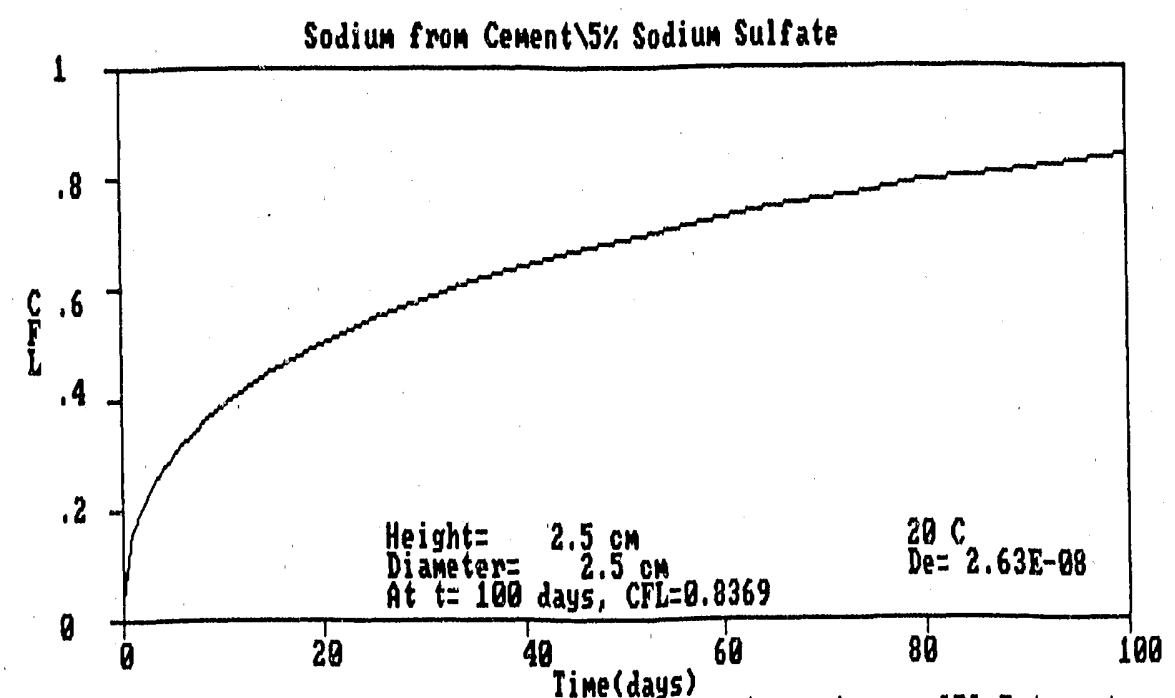

Press [P] Plot another line, [C] Continue to next species or [R] Return to menu

\section{EXPLANATION}

With the information from SCREENS 2 and 3 and the data held in the working memory, the program will generate a plot of cumulative fraction leached (CFL) versus time (in days). The series title of the data set appears at the top. The $X$-axis extends to the time ( $\mathrm{t}$ ) defined in SCREEN $2 \mathrm{~L}$ and the CFL calculated for that time period is also displayed. The height, diameter, species name and the diffusion coefficient are displayed on the plot. The program will automatically produce graphs for each data species in the file. 


\title{
SCREEN 4L * Continued
}

\begin{abstract}
A prompt is displayed under the graph stating "Press [P] Plot another line, [C] Continue to next species or [R] Return to Menu." Press only the letter; no brackets are needed.
\end{abstract}

Press $\mathbf{R}$ to return to the main menu.

Press $\mathbf{C}$ to return to SCREENS $2 \mathrm{~L}$ and $3 \mathrm{~L}$ using the parameters for the next species in the data set held in the working memory.

Press $\mathbf{P}$ to advance to SCREEN 5L. 


\section{SCREEN 5L}

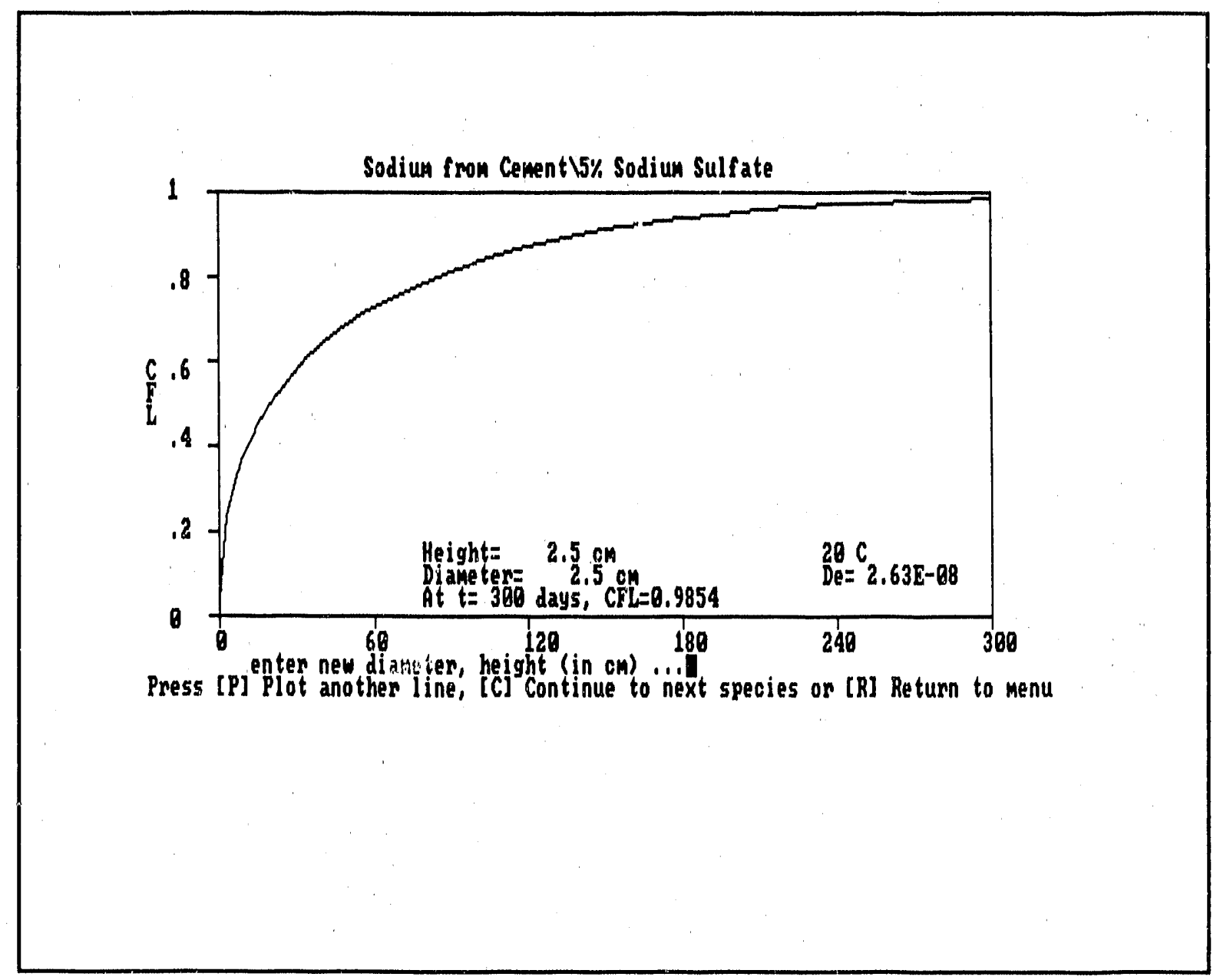

\section{EXPLANATION}

After pressing $\mathbf{P}$, a prompt appears under the graph asking the user to enter new values for the diameter and height (in centimeters) of the sample to be modeled. Enter new values separated by a comma or press ENTER to use the old values. 


\section{SCREEN 6L}

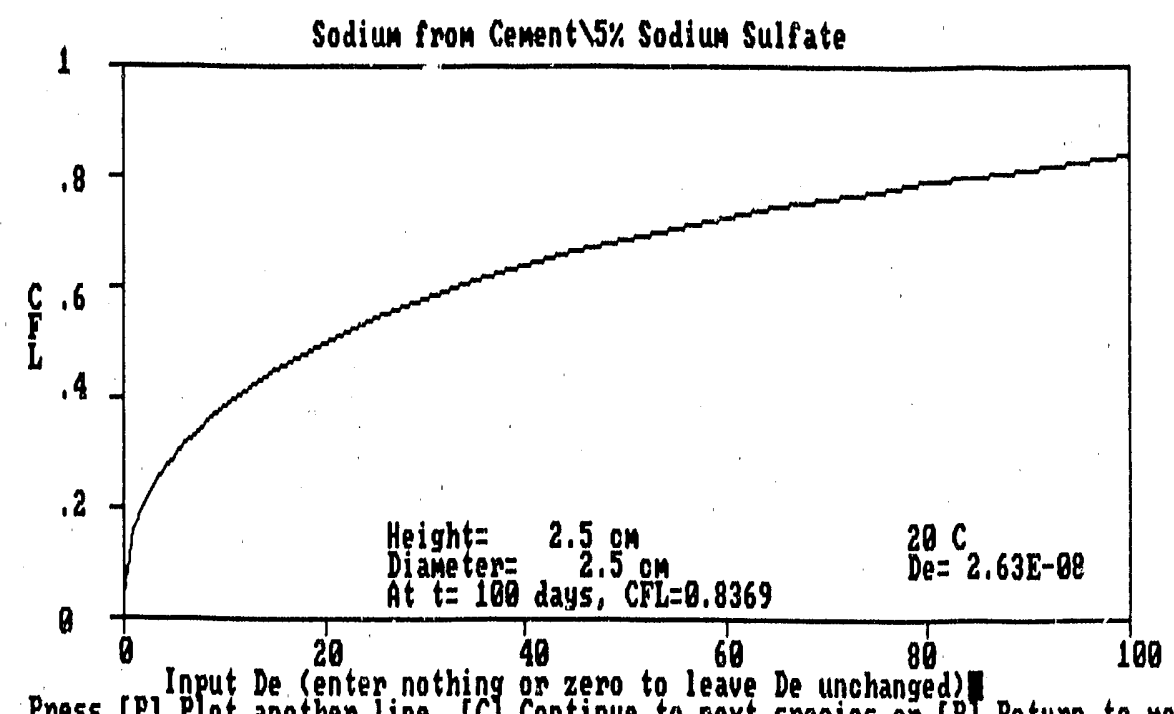

Press [P] Plot another line, [C] Continue to next species or [R] Return to menu

\section{EXPLANATION}

The prograrn now asks for a new value for $D_{e}$, the diffusion coefficient. A news value can be entered, and the old value can be retained by pressing ENTER. 


\section{SCREEN 7L}

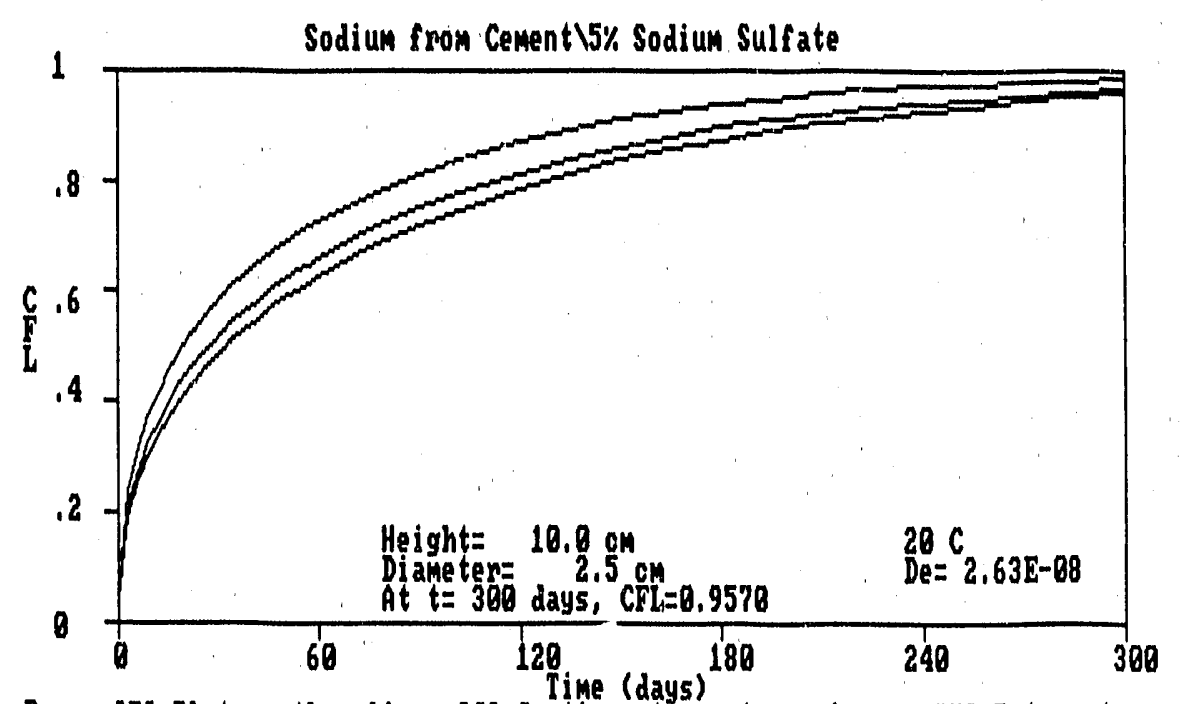

Press [P] Plot another line, [C] Continue to next species or [R] Return to menu

\section{EXPLANATION}

When the request for a value of $D_{0}$ is answered, the new curve is plotted on the previous graph. The latest values for diameter, height, $D_{0}$, and CFL are displayed. 


\section{SECTION M}

\section{EXIT PROGRAM}




\section{SCREEN $1 M$}

Use the cursor keys to highlight your choice, then press enter to choose it.

Enter new (raw) data

Enter data as CFL vs Time

Retrieve a data set

store a data set

Print a final data set

Edit data

To add data to a file

Plot data

Plot comparison

Determine De at Temp.

Extend modeled curve

Exit program

Developed for

DOE National Low-Level Waste Management Program by

Brookhaven National Laboratory

Low-Level Waste Research Group

Upton, N.Y. 11973

Press Fl for help and definitions

\section{EXPLANATION}

This function is used to exit from the "ALT" program. 


\section{SCREEN 2M}

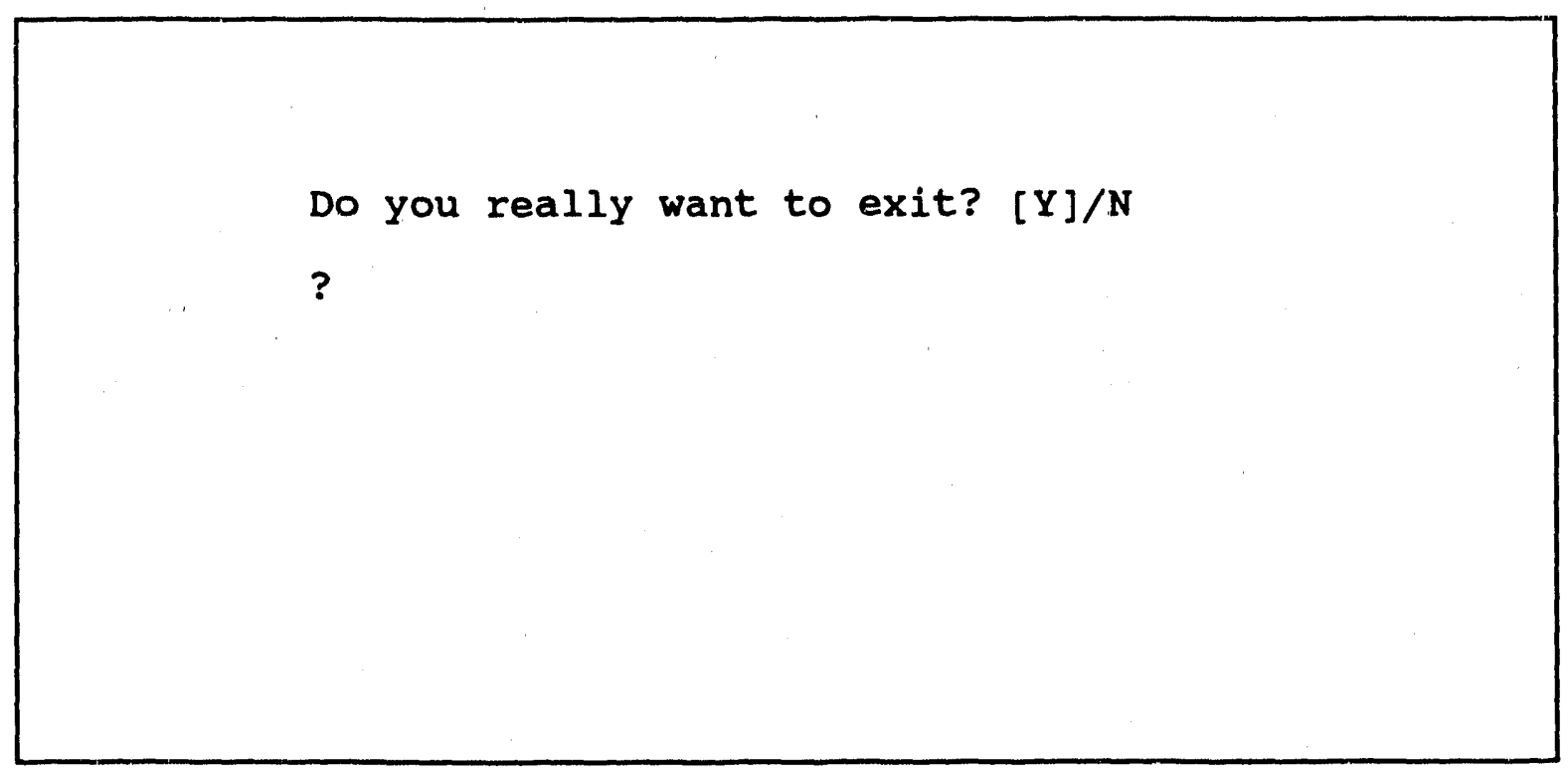

\section{EXPLANATION}

Answer "Y" or press "ENTER" to leave the program; the computer will return to DOS. If you press "N," the program returns to the MAIN MENU. 


\section{APPENDIX A}

\section{THEORY}

Models based on mass transport theory that have been validated with experimental data are regarded as an excellent means of estimating the amounts of material released by solidified waste. Critical to the evaluation of observed leaching results is the comparison with the theoretical models for diffusional release. The simplest models used for comparison with experimental results are for bulk diffusion.

\section{Bulk Diffusion}

The mathematical theory of transport by diffusion from solids is based on Fick's hypothesis that the diffusion rate is proportional to the concentration gradient[1,2]. The fundamental partial differential equation for diffusion is:

$$
\frac{\partial C}{\partial t}--D \cdot \nabla^{2} C
$$

where $\mathrm{C}$ is the concentration of the species, $t$ is time, and $D_{c}$ is the effective diffusion coefficient in porous media.

Mathematical solutions to the transport equation for diffusion have been applied to the leaching of radionuclides from waste solids[3,4]. The exact form of the solution to the mass transport equation of diffusion depends on the initial and boundary conditions of the problem. The semi-infinite media and the finite shape models are the most direct and generally used.

\section{Semi-Infinite Media Model}

A simple case is that of a semi-infinite solid with a constant diffusion coefficient where ideally the cumulative fraction released from a porous medium is predicted as:

$$
C F L-\frac{\sum a_{n}}{A_{0}}-2 \frac{s}{V}\left[\frac{D_{g}}{\pi}\right]^{1 / 2}
$$


where $a_{n}$ is the total amount of radioactive material released in all leaching periods up to time, $t, A_{0}$ is the initial amount of radioactive material, $V$ is the waste form volume, $S$ is the waste form surface area, $D_{c}$ is the effective diffusion coefficient. The semi-infinite model has been used to model leaching behavior when the cumulative fraction leached $\left(\mathrm{CFL}=\Sigma \mathrm{a}_{\mathrm{n}} / \mathrm{A}_{0}\right)$ is less than 0.20 or $20 \%$ release.

\section{Finite Shape Models}

Laboratory samples are often leached well beyond the $20 \%$ maximum limit for the semi-infinite model. Consequently, the analytical solution for diffusion from a finite shape must be used to account for depletion of the source term. Cylindrical shaped waste forms are commonly used. Hence the exact solution for diffusion from a finite cylinder using a constant diffusion coefficient, as described by Nestor, is appropriate. The solution of the mass transport equation for a constant diffusion coefficient, a uniform and homogeneous solid and zero surface concentration during leaching is:

$$
C F L=\frac{\sum a_{n}}{A_{0}}-1-\frac{32}{\pi^{2}} \sum_{n=1}^{\infty} \sum_{m=1}^{\infty} \frac{e^{-t D_{c}\left[\beta_{-}^{2}+(2 n-1)^{2} x^{2} \mu \mu^{2}\right]}}{(2 n-1)^{2}\left(\beta_{n}\right)^{2}}
$$

where $D_{e}=$ effective diffusion coefficient $\left(\mathrm{cm}^{2} / \mathrm{s}\right), \ell=$ half height of the cylinder $(\mathrm{cm}), a_{n}$ is the cumulative leached activity, $A_{0}=$ initial total activity, $m=$ positive roots of Bessel function where, $J_{0}\left(r \beta_{m}\right)=0$.

The finite cylinder model provides a guideline to determine if diffusion continues to consistently operate as the release mechanism during leaching tests. However, the use of equation (3) has been frustrated by the slow convergence of the double infinite serics. Consequently, several investigators have presented alternative methods of using the difficult to evaluate double infinite series solution for diffusion from a finite cylinder $[4,5,6,7]$. Pescatore's technique transformed the double series into a rapidly converging form for which the error associated with the truncation of each series can be evaluated. 
For finite cylinder waste forms of height $\mathbf{H}$ and radius $\mathbf{R}$, one uses instead the double series expression:

$$
C F L-\sum a_{n} / A_{0}-(1-b)\left[1-32 / \pi^{2} S_{p}(t) S_{c}(t)\right]+b
$$

with:

$$
S_{p}(t)-\sum_{n=1}^{\infty} \frac{\exp \left\{-[(2 n-1) \pi / H]^{2} D_{e} t\right\}}{(2 n-1)^{2}}
$$

and:

$$
S_{c}(t)-\sum_{m=1}^{\infty} \frac{\exp \left[-\left(\beta_{m} / R\right)^{2} D_{c} t\right]}{\beta_{m}^{2}}
$$

For the $S_{p}(t)$ series:

$$
S_{p}(t)-S_{p, N}(t)+E_{p, N}(t)
$$

with:

$$
\begin{gathered}
S_{p, N}(t)-\sum_{x=1}^{N-1} \frac{\exp \left[-(2-1)^{2} \gamma^{2}(t)\right]}{(2 n-1)^{2}}+\frac{N}{(2 N-1)^{2}} \exp \left[-(2 N-1)^{2} \gamma^{2}(t)\right] \\
-(\sqrt{ } \pi / 2) \gamma(t) \operatorname{efrc}[(2 N-1) \gamma(t)] \\
\gamma(t)=\frac{\pi\left(D_{e} t\right)^{1 / 2}}{H}
\end{gathered}
$$


and:

$$
0<E_{p, N}(t)<\frac{1}{6(2 N-1)}\left[\gamma^{2}(t)+2 /(2 N-1)^{2}\right] \exp \left[-(2 N-1)^{2} \gamma^{2}(t)\right]
$$

and for the $S_{c}(t)$ series:

$$
S_{c}(t)-S_{c, M}(t)+E_{c, w}(t)
$$

with:

$$
\begin{gathered}
S_{c, \mu}(t)-\sum_{m=1}^{M-1} \frac{\exp \left[-\beta_{M} \theta^{2}(t)\right]}{\beta_{M}^{2}}+\frac{1}{\beta_{M} f_{M}}+\frac{1}{2 \beta_{M}^{2}} \exp \left[-\beta_{M}^{2} \theta^{2}(t)\right] \\
\frac{-\sqrt{\pi \theta(t)}}{f_{M}} \operatorname{erfc}\left[\beta_{M} \theta(t)\right] \\
f_{M}-\pi-1 /\left(8 \pi M^{2}\right), \quad M>>1 \\
\theta(t)-\frac{(D t)^{1 / 2}}{R}
\end{gathered}
$$

and:

$$
E_{c, M}(t)<\frac{\pi}{6 \beta_{M}}\left[\theta^{2}(t)+\frac{1}{\beta_{M}^{2}}\right] \exp \left[-\beta_{M}^{2} \theta^{2}(t)\right]
$$

In practice, an intercept term, b, which has been added to equation (4), can be used to improve the fit of the model since extrapolation of observed results to $t=0$ does not necessarily pass exactly through $C F L=0$. For low-level waste forms this intercept, $b$, is often small $\left(<0.5 \% \mathrm{~A}_{0}\right)$ and has been referred to as the instantaneous wash-off term for extraneous surface material. 
The ALT computer program uses the semi-finite media model when CFL $<0.0124$, and the finite cylinder model (Equation 4) for greater CFL values. This is done to provide the minimum error in modeling results. The program also includes an intercept term that allows for instantaneous wash off.

\section{Diffusion Plus Partitioning of the Source Term}

Through our analysis of experimental data it has become apparent that the finite cylinder model alone cannot adequately describe releases from many types of materials. Several physical and chemical mechanisms may take place within and on the surfaces of waste forms that can result in curves of CFL vs time becoming asymptotic before $\mathrm{CFL}=1.00$ (100\% release). The precise nature of these processes is material specific and can include:

- $\quad$ physical adsorption of ions onto surfaces,

- substitution of a proportion of a species in the lattice structure of a new phase, and

- multiple sources of wastes leading to species being present in two or more chemical forms, having different solubilities.

In all of these cases the effect is similar in that the species of interest is partitioned between a fraction that is available for leaching and a fraction that is not. The partitioning process is essentially a reduction of the source term $\left(\mathrm{A}_{0}\right)$, with the assumption that partitioning results in a fraction of the species that is not released, or is released at a rate that is so low that it is not detectable. This model has been used for a variety of elements leached from several types of cement-based waste forms and was often able to provide goodness-of-fit $\left(E_{r}\right)$ values that were far superior to the diffusion model. An example of the effectiveness of this model is shown in Figure A1, in which data from a leaching experiment is shown with curves generated by the model for diffusion from a finite cylinder and from the model for diffusion plus partitioning.

The partition model uses the model for diffusion from a finite cylinder (or from a semi-infinite cylinder if the CFL $<0.0124$ ), but alters the result by reducing the original source term so that the cumulative fraction leached is determined as follows:

$$
C F L=\frac{\sum a_{N}}{A_{0} P}-2 \frac{S}{V}\left[\frac{D_{e} t}{\pi}\right]^{1 / 2}
$$


where $a_{n}$ is the total amount of radioactive material released in all leaching periods up to time $t, A_{0}$ is the initial source term which is the amount of radioactive material originally present in the solid sample, $\mathbf{P}$ is the source term partitioning factor, which is $0<P<1, V$ is the volume of the solid sample, $S$ is the geometric surface area of the solid sample, and $D_{e}$ is the effective diffusion coefficient. There is, therefore, a fraction of activity that is not leached but retained on the solid:

$$
F R=\frac{A_{0}-A_{0} P}{A_{0}}-1-P
$$

where FR is the fraction of activity retained on the solid.

Certain caveats are necessary in using this model. Embodied in it is the assumption that the partitioning is absolute in the sense that the material unavailable for leaching will never leach out of the solid. This is not necessarily so. For example, releases from some materials may be best described by a model containing two diffusion coefficients; one being a much greater rate than the other. This model has not yet been added to the ALT program. In short-term tests the partition model will closely mimic the model using two diffusion coefficients, but extrapolations to longer times could be misleading. Therefore, no extrapolations are currently allowed when the partitioning model is used. Moreover, when the partitioning model is used to help define leaching mechanisms, we strongly recommended that other experimental evidence (in addition to the leach test data) should be used to verify the process of partitioning. In many cases, a simple batch-type adsorption test would suffice. 

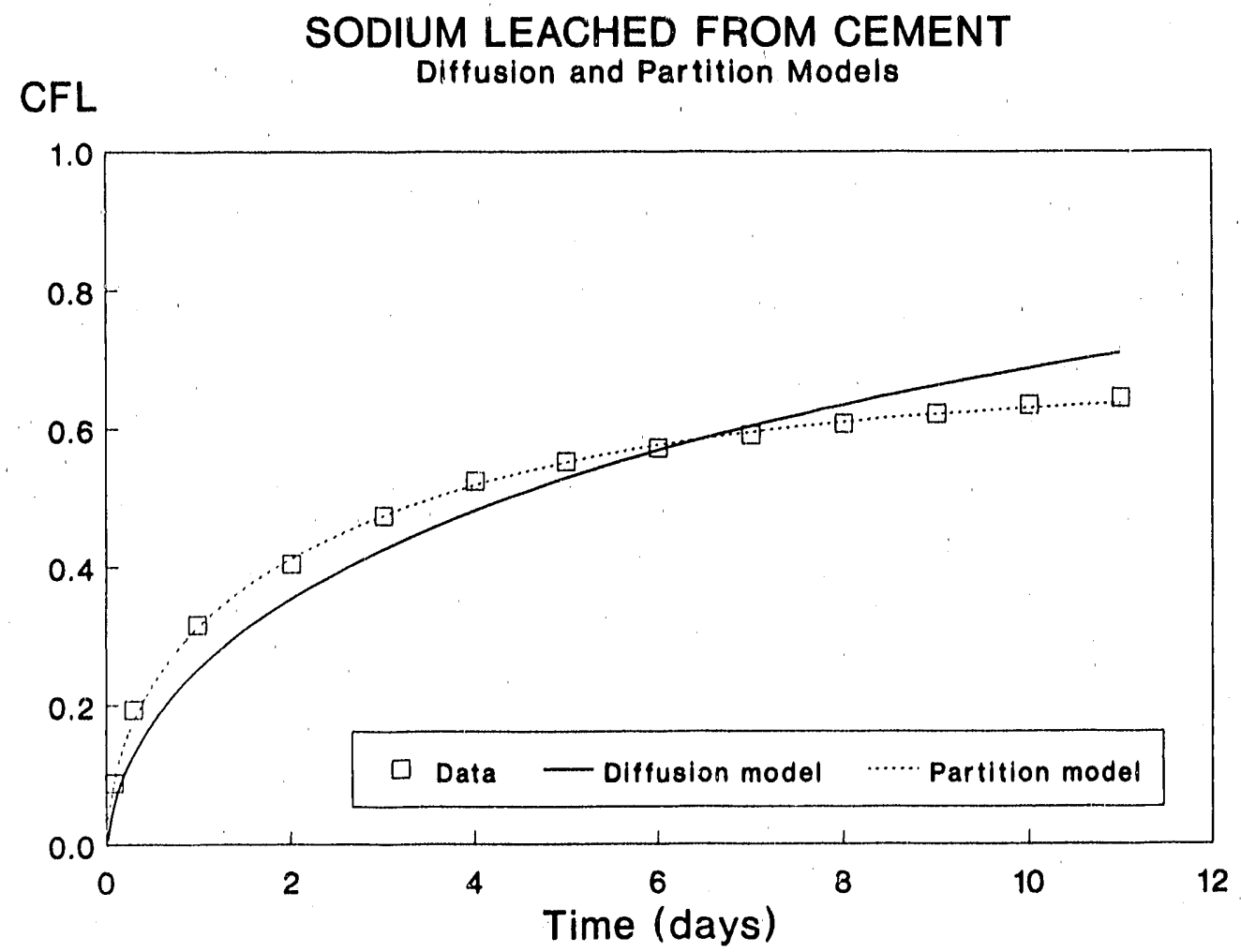

Figure A1. Experimental data as cumulative fraction leached, plotted as a function of time are shown as the squares. The finite cylinder diffusion model and the model in which the source term is partitioned are shown as lines fitted to the data. Date is for sodium leaching from portland cement containing sodium sulfate.

\section{Dissolution}

Solubility limited releases in leaching experiments are the result of two related processes:

- $\quad$ solubility limits constraining the dissolution of a phase inside the waste form, thereby reducing the leach rate and

solubility acting on the leachate, as a new phase forms external to the waste form, to limit concentrations in the leachate.

In the first case, the shape of the CFL vs time plot can take two forms. If the dissolution rate is great compared to the diffusion rate out of the waste form, release will be controlled by diffusion and the plot will be a typical curve. If, however, the dissolution rate is small compared to the diffusion rate, then the plot will have a linear shape, indicating dissolution as the rate controlling mechanism. 
In the second case, the plot of CFL vs time will also be linear as a result of a reaction between the components of the leachate and some external material generating a precipitate, leaving only a small amount of the component in solution. This will be the case when the reaction generating the precipitate is rapid compared to the rate of transport out of the solid. A typical example of this is as follows; the concentration of calcium in leachate generated from a cement sample is limited to about $6 \mathrm{mg} / \mathrm{L}$ by the solubility of several calcium carbonate minerals that form as precipitates in the leachate and as coatings on the sample. The precipitate is the reaction product of calcium and hydroxide ions leached from the cement and carbon dioxide drawn into solution from the air.

The dissolution model incorporated into the accelerated leach test computer program cannot be used to make long-term predictions, instead it is intended for use as an indicator of processes (such as precipitation) influencing the leach test. The concept involved is that the concentrations of the species that are solubility controlled will be the same at all leaching intervals. This means that the incremental fraction leached will be the same for all intervals and the cumulative fraction leached will increase by the same amount at each interval, producing the linear form of the plot showing CFL vs time. A plot of IFL vs time is a horizontal line. In reality a plot of IFL vs time shows a distribution around a mean value of IFL, which can be described by a statistical analysis of the data. For the ALT program , the coefficient of variation is used to evaluate the goodness-of-fit of the experimental data compared to the model (the average). The equation to calculate the coefficient of variation is shown below and is a way of normalizing the standard deviation so that it can be expressed as a percentage of the mean:

$$
V=\frac{s \times 100}{\bar{x}}
$$

where $S$ is the standard deviation and $\bar{x}$ is the mean IFL, and $V$ is the coefficient of variation. The standard deviation (S) is calculated as follows:

$$
s-\sqrt{\frac{\sum_{i-1}^{n-1}(X-\bar{x})^{2}}{n-1}}
$$

where $\mathbf{n}$ is the number of data points and $\mathrm{X}$ is the IFL of individual data points. The first two data points are not included in the calculation because experimental data indicate that the time intervals for those samplings are two small to allow solubility limits to be reached. A coefficient of variation of $10 \%$ indicates that the data fits this linear model and that solubility limits control releases. 


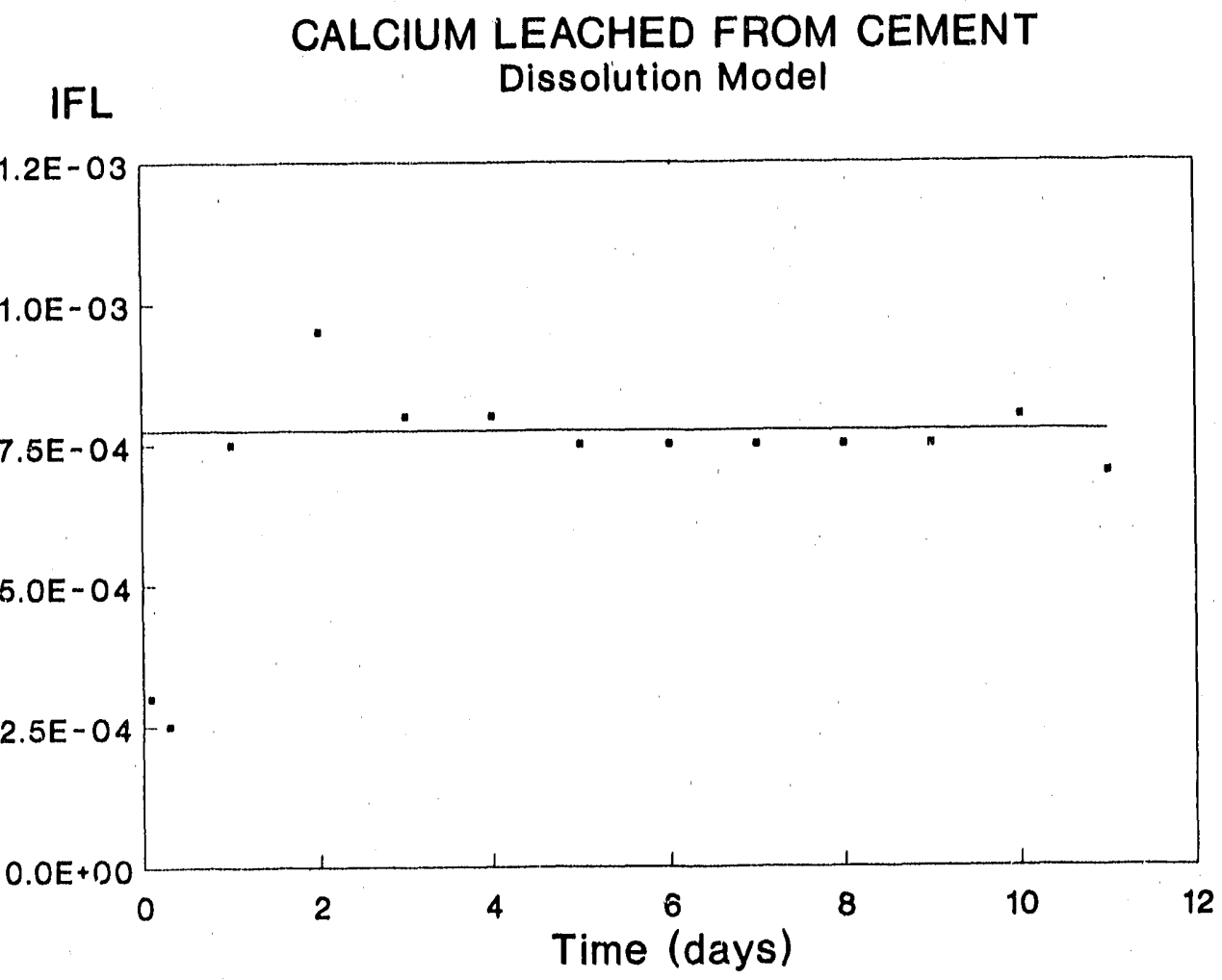

Figure A2 A plot of incremental fraction leached (IFL) as a function of time illustrates solubiliby' limited released. IFL is described by a means value with a distribution about that mean. The first two data points are ignored because the time intervals between samplings are too short to be useful in this model. 


\section{REFERENCES}

\section{APPENDIX A}

1. Crank, J., The Mathematics of Diffusion, Claredon Press, Oxford (1975).

2. Godbee, H.W., E.L. Compere, D.S. Joy, A.H. Kibbey, J.G. Moore, C.W. Nestor, Jr., O.U. Anders and R.M. Neilson, Jr., "Application of Mass Transport Theory to the Leaching of Radionuclides of Waste Solids," Nuclear and Chemical Waste Management, Vol. 1, pp. 29-35 (1980).

3. Machiels, A.J. and C. Pescatore, "Modeling of Waste Form Leaching, Part I Status of Leaching Modeling," Technical Report, UILU-ENG-82-5319, University of Illinois (March 1982).

4. Thomas, G.F., "Diffusional Release of a Single Component Material from a Finite Cylindrical Waste Form," Ann. Nucl. Energy, V14.283 (1987).

5. Ganapol, B.D., "Evaluation of the Material Release from a Cylindrical Waste Form via Laplace Transforms," R.G. Post (editor), Waste Management 88, Vol. 1, pp.493-499 (1988).

6. Nestor, C.W., "Diffusion from Solid Cylinders," ORNL/CSD/TM 84, Oak Ridge National Laboratory, Oak Ridge, TN (1980).

7. Pescatore, C., "Improved Expressions for Modeling Diffusive, Fractional Cumulative Leaching from Finite-size Waste Forms, "Waste Management, Vol. 10, pp. 155-159 (1990). 


\section{APPENDIX B}

\section{GLOSSARY}

Acceleration Factor. Ratio calculated as the time required to reach a certain Cumulative Fraction Leached for an accelerated leach test divided by the time required by a reference leach test to reach that same Cumulative Fraction Leached.

Accelerated Leach Test. A leach test procedure that provides greater release rates than those obtained through standard leach tests. Test conditions, such as elevated temperature and increased volume of leachant can be used to increase release rates without changing the mechanisms of leaching.

Cumulative Fraction Leached (CFL). The sum of the fractions leached during all sampling intervals calculated as:

$$
\mathrm{CFL}=\Sigma \mathrm{a}_{\mathbf{n}} / \mathrm{A}_{0}
$$

and assuming no radioactive decay.

Diffusion Coemicient. (Diffusivity) Based on Fick's Laws for diffusion, the diffusion coefficient is the ratio of the rate of transfer of a diffusing substance through the unit area of a section to the concentation gradient measured normal to the section. The diffusion coefficient is symbolized by $D$.

Diffusion Model. Computer algorithm using diffusion theory to describe leaching. Two diffusion models are used in the ALT program: diffusion from a semi-infinite medium and diffusion from a cylinder.

Dissolution Model. Computer algorithm that statistically analyses IFL values throughout an experiment by calculating a mean and the distribution about the mean. A consistent value of IFL indicates solubility controls on the concentration of the species of interest in the leachate.

Effective Diffusion Coeficient. (Effective Diffusivity) The diffusion coefficient that results from diffusion as it is modified by other processes (e.g. adsorption) or physiral constaints (tortuosity and constrictivity). The effective diffusion coefficient is symbolized by $D_{e}$.

Finite Cylinder. (Finite Medium) A bounded body for which the diffusion equation can be solved. 
Goodness-of-Fit. An expression indicating how closely a model generated curve comes to representing a data curve. In the ALT program, goodness-of-fit is represented quantitatively (for the diffusion model and the partition model) by the value $E_{R}$. This is the sum-of-the-residuals expressed as a percentage of the final CFL of the experimental data. For the dissolution model, goodness-of-fit is indicated by the size of the distribution about a mean value of CFL. That distribution is described by the standard deviation, expressed as the coefficient of variation.

Incremental Fraction Leached (IFL). The fraction leached of a species of interest during a single sampling interval calculated as:

$$
I F L=a_{n} / A_{0}
$$

and assuming no radioactive decay.

Leachant. The liquid that contacts the specimen during a leach test or contacts a waste form in the disposal environment.

Leachate. The leachant after contacting the specimen or the waste form.

Leaching. The process (or processes) by which mass transport from a solid to a liquid takes place.

Leaching Interval. The length of time during which a given volume of leachant is in contact with a specimen.

Leaching Mechanism. The process which controls the rate of mass transport out of a specimen during leaching.

Low-Level Radioactive Waste. Waste that contains radioactivity and is not high-level waste, transuranic waste, or spent nuclear fuel as defined by Department of Energy Order 5820.2A. Test specimens of fissionable material irradiated for research and development only, and not for production of power or plutonium, may be classified as low-level waste, provided the concentration of transuranics is less than 100 $\mathrm{nCi} / \mathrm{g}$. 
Partition Model. Computer algorithm, based on the diffusion models, that reduces the source term of the model. This implies that the species-of-interest is contained in two fractions: one reachable and the other not leachable.

Reference Leach Test. A leach test conducted under defined conditions where the results are used as a standard against which the results of other leach tests (e.g. accelerated) are compared.

Semi-dynamic Leach Test. A leach test method that exposes the specimen to fresh leachant on a periodic schedule.

Semi-infinite Medium. A body, used in diffusion theory, the outer boundary of which is effectively at an infinite distance from the inner region.

Solidification Agent. A material used to process waste into a solid, stable waste form.

Source Term. The original concentration of a species of interest in a specimen or a waste form, before leaching.

Sum-of-the-Residuals. The difference, taken at each data point, between the CFL of the model and the CFL of the data, summed through the entire data set.

Surface Area. For purposes of this test method, surface area is defined as the geometric surfiuie area of a specimen calculated from its measured dimensions.

Volume. For purposes of this test method, volume is defined as the volume of a specimen calculated from its measured dimensions.

Waste Form. A stable, solid body formed by the waste and solidification agent and meeting specifications for disposal. 

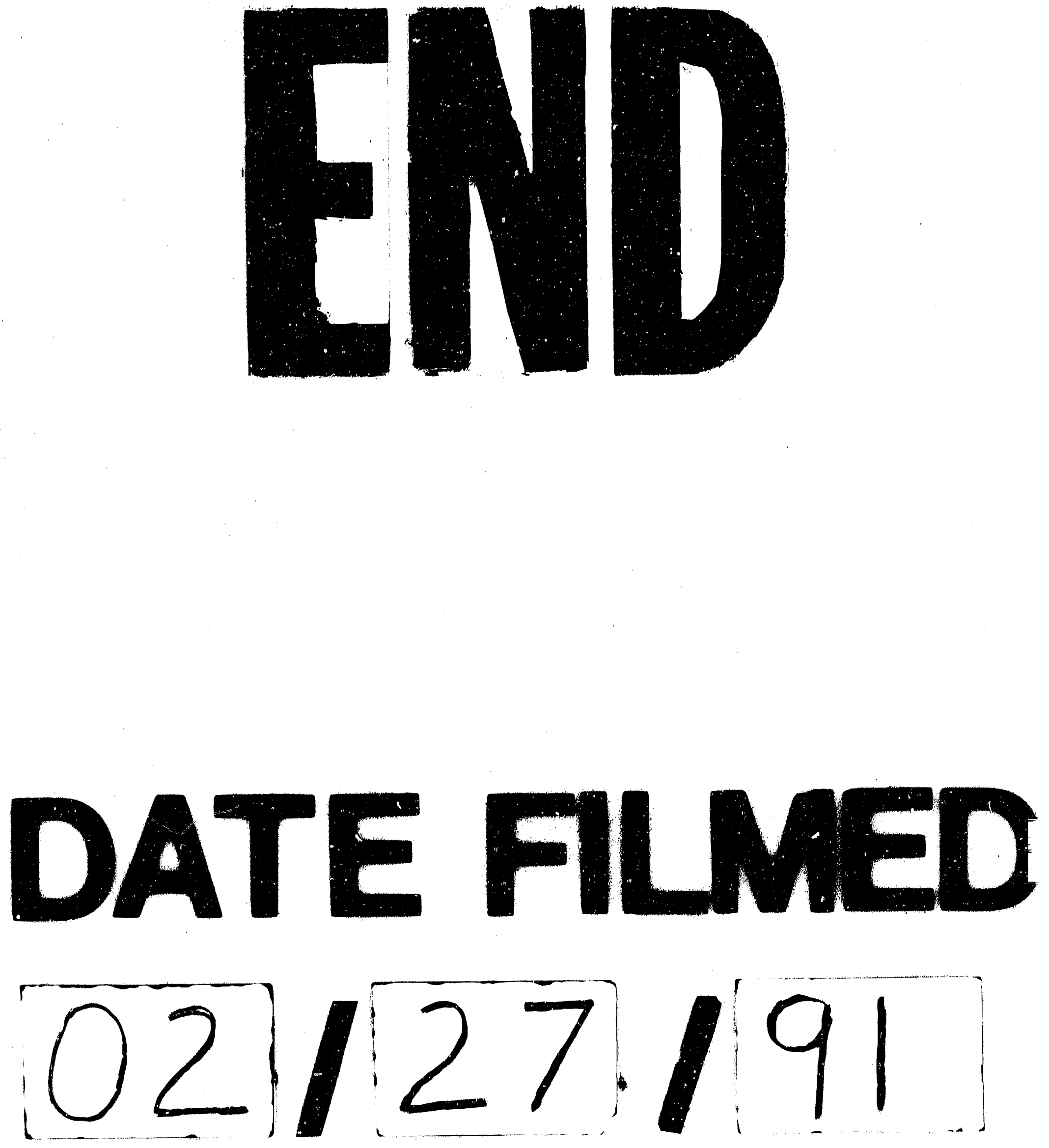
